\title{
Low Complexity Normal Bases
}

\author{
by \\ David Thomson, B.Math \\ A thesis submitted to \\ the Faculty of Graduate Studies and Research \\ in partial fulfillment of \\ the requirements for the degree of \\ Master of Science,
}

School of Mathematics and Statistics

Ottawa-Carleton Institute for Mathematics and Statistics

Carleton University

Ottawa, Ontario, Canada

(C)Copyright

2007, David Thomson 
To the number $\pi$, may you always be curiously identically equal to three. 


$\begin{array}{ll}\begin{array}{l}\text { Library and } \\ \text { Archives Canada }\end{array} & \begin{array}{l}\text { Bibliothèque et } \\ \text { Archives Canada }\end{array} \\ \begin{array}{l}\text { Published Heritage } \\ \text { Branch }\end{array} & \begin{array}{l}\text { Direction du } \\ \text { Patrimoine de l'édition }\end{array} \\ \begin{array}{l}\text { 395 Wellington Street } \\ \text { Ottawa ON K1A ON4 }\end{array} & \begin{array}{l}\text { 395, rue Wellington } \\ \text { Ottawa ON K1A ON4 } \\ \text { Canada }\end{array}\end{array}$

Your file Votre référence ISBN: 978-0-494-33713-4 Our file Notre référence ISBN: 978-0-494-33713-4

NOTICE:

The author has granted a nonexclusive license allowing Library and Archives Canada to reproduce, publish, archive, preserve, conserve, communicate to the public by telecommunication or on the Internet, loan, distribute and sell theses worldwide, for commercial or noncommercial purposes, in microform, paper, electronic and/or any other formats.

The author retains copyright ownership and moral rights in this thesis. Neither the thesis nor substantial extracts from it may be printed or otherwise reproduced without the author's permission.
AVIS:

L'auteur a accordé une licence non exclusive permettant à la Bibliothèque et Archives Canada de reproduire, publier, archiver, sauvegarder, conserver, transmettre au public par télécommunication ou par l'Internet, prêter, distribuer et vendre des thèses partout dans le monde, à des fins commerciales ou autres, sur support microforme, papier, électronique et/ou autres formats.

L'auteur conserve la propriété du droit d'auteur et des droits moraux qui protège cette thèse. $\mathrm{Ni}$ la thèse ni des extraits substantiels de celle-ci ne doivent être imprimés ou autrement reproduits sans son autorisation.
In compliance with the Canadian

Privacy Act some supporting forms may have been removed from this thesis.

While these forms may be included in the document page count, their removal does not represent any loss of content from the thesis.
Conformément à la loi canadienne sur la protection de la vie privée, quelques formulaires secondaires ont été enlevés de cette thèse.

Bien que ces formulaires aient inclus dans la pagination, il n'y aura aucun contenu manquant.

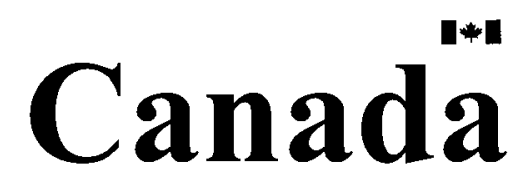




\section{Abstract}

We develop an efficient algorithm to enumerate and examine all normal bases over finite fields of characteristic two and degrees up to $n=39$. This improves on previous tables given in [22] which search to $n=30$. We create a table of minimum complexities for $n \leq 607$ using our new data and theorems from the literature. We conjecture on the average and variance of the distribution of complexities in a finite field. We further conjecture that there is no constant $k$ such that the minimum complexity of a normal basis of $\mathbb{F}_{2^{n}}$ over $\mathbb{F}_{2}$ is bounded below by $k n$.

We also give a new construction of low complexity normal bases for finite fields of any characteristic. We do this by projecting to subfields using the trace function from extensions where an optimal normal basis is known to exist. This is performed for both Type I and Type II optimal normal bases. We also examine the dual bases of our construction and give their resulting complexities. We compare the five fields recommended by NIST [33] for elliptic curve cryptography to fields with similar characteristics where we apply our construction and note that if our fields are cryptographically strong they admit a much lower complexity than the given fields. 


\section{Acknowledgements}

First, I must acknowledge my defense committee and thank them for all of their comments and insights. The committee includes, from the School of Mathematics and Statistics at Carleton University: Lucy Campbell, Daniel Panario and Steven Wang, and Ali Miri from the Department of Mathematics and Statistics at the University of Ottawa.

Special thanks to the School of Mathematics and Statistics at Carleton University for their generous offer of the Herbert and Erwin Kreysig Scholarship. Thanks to the faculty including inspiring professors and researchers David Amundsen, Paul Mezo, Michael Moore, Daniel Panario, John Poland, Steven Wang and many others. Also, thanks to the staff, particularly Valerie Daley who takes a special pride in her students.

Thanks again to Daniel Panario along with Maria Christopoulou, Theo Garefalakis, Ariane Masuda and Lucia Moura for their joint effort on the new results presented in Chapters 3 and 4 . The work in Chapter 3 was first performed during an NSERC USRA award. The work in Chapter 4 was first discussed at the workshop "Polynomials over Finite Fields and Applications" at the Banff International 
Research Station in November, 2006.

Lastly, I must thank the friends I've had throughout my stay at Carleton University. I know I will miss a few names but some of these very special people are Amanda Ager, Karin Arikushi, Chantal Bilton, Amy Boles, Lyne Sleiman, Jenna Tattersall, Veronica Tomporowski and Alex Coassin (immortalized as "V and Cosine"), Josh Ulla and Colin Weir. 


\section{Contents}

Abstract

1 Introduction 1

2 Normal Bases in Finite Fields 4

2.1 Finite Fields . . . . . . . . . . . . . . . . . 4

2.1.1 Traces and Bases of Finite Fields . . . . . . . . . . . . . 10

2.2 Introduction to Normal Bases . . . . . . . . . . . . . 16

2.2.1 Arithmetic Using Normal Bases . . . . . . . . . . . . 20

2.3 Optimal Normal Bases . . . . . . . . . . . . . . . 24

2.3.1 Characterization of Optimal Normal Bases . . . . . . . 25

2.3.2 Determination of all Optimal Normal Bases . . . . . . 29

2.4 Distribution of Normal Bases . . . . . . . . . . . . . . 36

2.5 Constructions of Normal Bases . . . . . . . . . . . . . . . 41

2.6 The Dual of a Normal Basis . . . . . . . . . . . . . . . . 46

3 Distribution of Complexities $\quad 52$

$\mathrm{V}$ 
3.1 Exhaustive Search of Normal Bases . . . . . . . . . . . 53

3.1.1 Description of the Algorithms ............ 53

3.1 .2 Gray Codes ....................... 55

3.1.3 StandardNCD and GrayCodeNCD Algorithms . . . . . . 56

3.1.4 Karatsuba Multiplication Scheme .......... 58

3.1.5 Analyses of the Algorithms . . . . . . . . . . 60

3.2 Results............................ 62

3.2.1 Running Time Comparison . . . . . . . . . . . . 62

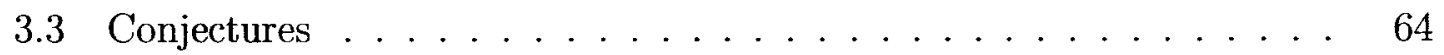

4 Traces of Optimal Normal Bases $\quad 68$

4.1 The Trace of a Normal Element . . . . . . . . . . . . 69

4.2 Main results . . . . . . . . . . . . . . . . . 69

4.2.1 Type I Optimal Normal Bases: $q$ odd . . . . . . . . . 70

4.2 .2 Type I Optimal Normal Bases: $q$ even . . . . . . . . . . 75

4.2 .3 Type II Optimal Normal Bases . . . . . . . . . . . . 78

4.3 The Dual of the Trace of an Optimal Element . . . . . . . . 80

4.4 Existence of Optimal Extensions . . . . . . . . . . . . . 88

4.5 Summary and Comparisons . . . . . . . . . . . . . . . 89

5 Conclusions $\quad 92$

5.1 Summary of Results . . . . . . . . . . . . . . . 92

5.1.1 Explanation of Tables in Appendices B and C . . . . . . . 94 
5.2 Further Work and Questions . . . . . . . . . . . . 95

$\begin{array}{ll}\text { A Optimal Normal Bases over } \mathbb{F}_{2} & 98\end{array}$

B Exhaustive Search of $\mathbb{F}_{2^{n}}, n<40 \quad 100$

C Complexities Found $40 \leq n \leq 607 \quad 102$

D Low-Complexity Subfields

Bibliography 


\section{Chapter 1}

\section{Introduction}

The choice of representation of elements in a finite field is critical for performing efficient calculations in the field. There are two standard bases used to represent a finite field: polynomial bases and normal bases.

A polynomial basis of $\mathbb{F}_{q^{n}}$ over $\mathbb{F}_{q}$ is given by $\left\{1, \alpha, \alpha^{2}, \ldots, \alpha^{n-1}\right\}$, where $\alpha$ is a root of an irreducible polynomial $f$ of degree $n$ over $\mathbb{F}_{q}$. In a polynomial basis, multiplication of elements becomes more efficient as the number of nonzero terms in $f$ decreases. For a discussion of polynomial bases and constructions of low-weight polynomials see, for example, $[13,18,21]$.

An alternate basis of $\mathbb{F}_{q^{n}}$ over $\mathbb{F}_{q}$ is given when an element $\alpha \in \mathbb{F}_{q^{n}}$ and its conjugates $\alpha^{q}, \alpha^{q^{2}}, \ldots, \alpha^{q^{n-1}}$ are linearly independent. A basis formed by the conjugates of $\alpha$ is called a normal basis. Normal bases are efficient for applications where large powers of elements are needed, such as in public key cryptography, but have the drawback that ordinary multiplication is computationally expensive. A measure of 
this expense is called the complexity of the normal basis. We examine methods and constructions for optimizing the complexity of a normal basis in a finite field.

We make every effort to make this work self-contained. In Chapter 2 we begin by giving some key results on finite fields. When feasible, theorems and definitions are given in generality and then narrowed to the scope of finite fields. Critical notions such as the trace of a field element are introduced. We give known results on normal bases that are necessary for all results we derive later. We give a discussion of normal basis arithmetic and a proof of the normal basis theorem. The complexity of a normal basis is introduced and we show the constructions of an optimal normal basis. We give Gao's original proof [10] that all optimal normal bases are equivalent to these constructions. We derive the number of normal bases in a finite field generally and give some computationally feasible bounds. We then give some previously known constructions of normal bases with given complexities. Finally, we discuss the dual of a normal basis, prove that this is again a normal basis, give necessary conditions for the existence of the dual of a normal basis and cite some results on the complexities of the dual of a normal basis.

We begin giving new results in Chapter 3. Some of the contents of this chapter are given in [27]. We develop an efficient algorithm to enumerate and examine all normal bases over finite fields of characteristic two and degrees up to $n=39$. This improves on previous tables given in [22] which search until $n=30$. We analyze the cost complexity of the algorithm and compare the run time of our algorithm with 
a non-optimized version. We use the data obtained by our search to explore the distribution of normal bases. We then create a table of minimum complexities for degrees $n \leq 607$ using our new data and theorems from the literature. We end by giving conjectures on distribution of normal elements in a finite field with respect to their complexities.

The results from Chapter 3 indicate experimentally that there is a very low probability that any randomized algorithm will provide normal bases with low complexity, and so in Chapter 4 we give a direct construction of normal bases in finite fields with low complexity. The contents of this chapter are outlined in [4]. We give our construction by projecting to subfields from fields which contain an optimal normal basis using the field trace function. This projection again yields normal bases with a computable (low) upper bound on their complexities. We also construct the dual bases of these projections and give upper bounds on their complexities. We discuss some deep questions on how large our field must reach to contain the desired extension as a subfield and also contain an optimal normal basis.

We give a summary of all results obtained in Chapter 5 . Section 5.2 concludes by posing some open problems which arose in conducting the research outlined above. The appendices present tables of data referred to in Chapters 3 and 4 . 


\section{Chapter 2}

\section{Normal Bases in Finite Fields}

The following assumes some basic knowledge of rings and fields. Some key results and definitions of field extensions and Galois theory are given for completeness.

\subsection{Finite Fields}

All definitions and theorems can be found in the text and exercises of Dummit and Foote's Abstract Algebra [6, Chapter 13-14], unless otherwise indicated. Some proofs are given for clarity.

Theorem 2.1.1. Let $f \in F[x]$ be an irreducible polynomial of degree $n$ over the field $F$ and let $K=F[x] /(f)$. Let $\theta \equiv x(\bmod f)$. Then, the powers

$$
1, \theta, \theta^{2}, \ldots, \theta^{n-1}
$$


are a basis for $K$ viewed as a vector space over $F$ and therefore $[K: F]=n$. We have

$$
K=\left\{a_{0}+a_{1} \theta+\cdots+a_{n-1} \theta^{n-1} ; \quad \text { with } a_{0}, a_{1}, \ldots, a_{n-1} \in \mathbb{F}\right\}
$$

and so $K$ consists of all polynomials of degree up to $n-1$ in $F[\theta]$. Addition in $K$ is defined by polynomial addition and multiplication in $K$ is defined by

$$
a(\theta) b(\theta)=r(\theta)
$$

where $a(x) b(x)=q(x) f(x)+r(x)$ for some $q, r \in F[x]$ where $\operatorname{deg}(r)<n$.

Theorem 2.1.2. Let $F$ be a field and let $f \in F[x]$ be an irreducible polynomial of degree $n$. Suppose $K$ is an extension field of $F$ containing a root $\alpha$ of $f$. Let $F(\alpha)$ denote the subfield of $K$ generated over $F$ by the element $\alpha$. Then $F(\alpha) \cong F[x] /(f)$ and

$$
F(\alpha)=\left\{a_{0}+a_{1} \alpha+\cdots+a_{n-1} \alpha^{n-1} ; \quad \text { with } a_{0}, a_{1}, \ldots, a_{n-1} \in F\right\} \subseteq K
$$

The following theorem is the keystone upon which Galois theory is built.

We now begin to characterize all finite fields. For the remainder of this discussion, we always assume that $p$ is a prime integer, and $q$ denotes the prime power $p^{n}$ for some integer $n \geq 1$.

Proposition 2.1.3. Let $\mathbb{F}$ be a finite field of characteristic $p$. Then $\mathbb{F}$ has $p^{n}$ elements 
for some integer $n \geq 1$.

Proof. Let $\mathbb{F}$ be a finite field of characteristic $p$. Then $\mathbb{F}$ has prime subfield isomorphic to $\mathbb{Z}_{p}$. In particular, $\mathbb{F}$ is a vector space over $\mathbb{Z}_{p}$ and so it has a basis $\alpha_{0}, \alpha_{1}, \ldots, \alpha_{n-1}$ over $\mathbb{Z}_{p}$ for some integer $n \geq 1$. Any element in $\mathbb{F}$ is of the form

$$
z_{0} \alpha_{0}+z_{1} \alpha_{1}+\cdots+z_{n-1} \alpha_{n-1} ; \quad \text { with } z_{0}, z_{1}, \ldots, z_{n-1} \in \mathbb{Z}_{p}
$$

and so there are $p^{n}$ such elements.

Definition 2.1.4. The extension field $K$ of $F$ is called a splitting field for the polynomial $f \in F[x]$ if $f$ factors completely into linear factors in $K$ and $f$ does not factor into linear factors in any subfield of $K$ containing $\mathbb{F}$.

Theorem 2.1.5. (Existence and Uniqueness of Splitting Fields) Let $f \in F[x]$. Then there exists a splitting field $E$ of $f$ over $F$ and any two splitting fields of $f$ over $F$ are isomorphic under an isomorphism which fixes $F$ and maps roots of $f$ into each other.

Lemma 2.1.6. Let $f \in F[x]$, then $f$ has a multiple root $\alpha$ if and only if $\alpha$ is also a root of $f^{\prime}$. In particular, $f$ is separable if and only if $\operatorname{gcd}\left(f, f^{\prime}\right)=1$.

Example 2.1.7. Consider $f(x)=x^{p^{n}}-x$ over $\mathbb{F}_{p}$, then $f^{\prime}(x)=p^{n} x^{p^{n}-1}-1=-1$ since $\mathbb{F}_{p}$ has characteristic $p$. Therefore $f^{\prime}$ has no roots and $f$ is separable.

Theorem 2.1.8. (Existence and Uniqueness of Finite Fields) For every prime $p$ and 
positive integer $n$, there is a finite field with $p^{n}$ elements. Any finite field with $p^{n}$ elements is isomorphic to the splitting field of $x^{p^{n}}-x$ over $\mathbb{F}_{p}$.

Proof. Let $n$ be a positive integer and let $f(x)=x^{p^{n}}-x$ over $\mathbb{F}_{p}$. By Example 2.1.7, $f$ is separable and so $f$ has exactly $p^{n}$ distinct roots. If $\alpha$ and $\beta$ are $\operatorname{roots}$ of $f$ then $f(\alpha)=0=f(\beta)$ so $\alpha^{p^{n}}=\alpha$ and $\beta^{p^{n}}=\beta$

Denote $\mathbb{F}$ as the set of roots of $x^{p^{n}}-x$ over $\mathbb{F}_{p}$. Then $\mathbb{F}$ is closed under addition, multiplication, and inversion, so $\mathbb{F}$ is a subfield of the splitting field of $f$ over $\mathbb{F}_{p}$. By the minimality of the splitting field, $\mathbb{F}$ is the splitting field of $x^{p^{n}}-x$ over $\mathbb{F}_{p}$. This shows that, for any positive integer $n$, there exists a finite field of degree $n$ over $\mathbb{F}_{p}$.

To prove uniqueness, let $\mathbb{F}$ be a finite field of characteristic $p$. If $\mathbb{F}$ has dimension $n$ over $\mathbb{F}_{p}$ then $\mathbb{F}$ has $p^{n}$ elements, and the multiplicative group of $\mathbb{F}, \mathbb{F}^{*}$ has order $p^{n}-1$. Therefore for any $\alpha \in \mathbb{F}^{*}, \alpha^{p^{n}-1}=1$, and so $\alpha^{p^{n}}=\alpha$ and $\alpha$ is a root of $f$. Since the splitting field of $f$ has $p^{n}$ elements, $\mathbb{F}$ is a splitting field of $f$ over $\mathbb{F}_{p}$. Uniqueness of finite fields then follows directly from uniqueness of splitting fields.

We now explore the structure of all subfields of a given finite field $\mathbb{F}_{q}$.

Lemma 2.1.9. Let $n, d$ be integers, then $d$ divides $n$ if and only if $x^{d}-1$ divides $x^{n}-1$

Proof. By the Division Algorithm, $n=q d+r$ for integers $q, r, 0 \leq r<d$. So $x^{n}-1=\left(x^{q d+r}-x^{r}\right)+\left(x^{r}-1\right)$. Then $x^{d}-1$ divides $x^{n}-1$ if and only if $x^{d}-1$ divides $x^{r}-1$ and $x^{d}-1$ divides $x^{q d+r}-x^{r}=x^{r}\left(x^{q d}-1\right)$. The first condition holds if and only 
if $r=0$. The second condition holds since $x^{q d}-1=\left(x^{d}-1\right)\left(x^{q}-x^{q-1}+\cdots-x+1\right)$, as a telescoping sum.

Lemma 2.1.10. Let $n, d$ be integers, then $d$ divides $n$ if and only if $a^{d}-1$ divides $a^{n}-1$, for any integer $a$.

Proof. The previous lemma holds over any polynomial ring so, in particular, it holds over the ring of integers $\mathbb{Z}$. By the previous lemma, $d$ divides $n$ if and only if $x^{n}-1=$ $\left(x^{d}-1\right) q(x)$ for some $q(x) \in \mathbb{Z}[x]$. Since the evaluation map is a homomorphism it follows that for any integer $a, d$ divides $n$ if and only if $a^{n}-1=\left(a^{d}-1\right) q(a)$ where $q(a) \in \mathbb{Z}$

Theorem 2.1.11. (Subfield Criterion) Let $\mathbb{F}_{p^{n}}$ be the finite field with $p^{n}$ elements. Every subfield of $\mathbb{F}_{p^{n}}$ has order $p^{d}$ for $d$ is a positive divisor of $n$. Conversely, if $d$ is a positive divisor of $n$, there is a unique subfield of $\mathbb{F}_{p^{n}}$ with $p^{d}$ elements

Proof. If $\mathbb{F}$ is a subfield of $\mathbb{F}_{p^{n}}$, then it is again a finite field of characteristic $p$ so therefore has $p^{d}$ elements for some integer $d \leq n$. We can view $\mathbb{F}_{p^{n}}$ as a finite dimensional vector space (say, of dimension $m$ ) over $\mathbb{F}$ and so $\mathbb{F}_{p^{n}}$ has a basis of $m$ elements $\left\{b_{1}, b_{2}, \ldots, b_{m}\right\} \subseteq \mathbb{F}_{p^{n}}$. Then every element in $\mathbb{F}_{p^{n}}$ is of the form $a_{1} b_{1}+a_{2} b_{2}+$ $\cdots+a_{m} b_{m}$ for $a_{1}, a_{2}, \ldots, a_{m} \in \mathbb{F}$. Therefore, $p^{n}=\left(p^{d}\right)^{m}$.

Conversely, if $d$ is a positive divisor of $m$ then, by the above lemma, $p^{d}-1$ divides $p^{n}-1$, for any prime $p$. Consequently $x^{p^{d}}-x$ divides $x^{p^{n}}-x$. Then every root of $x^{p^{d}}-x$ is a root of $x^{p^{n}}-x$. Therefore $\mathbb{F}_{p^{n}}$ contains a splitting field of $x^{p^{d}}-x$ as a 
subfield. If $\mathbb{F}_{p^{n}}$ contained two distinct subfields which split $x^{p^{d}}-x$ then there would be more than $p^{d}$ roots, which is a contradiction.

This gives a unique lattice of subfields for any finite field $\mathbb{F}_{p^{n}}$ over $\mathbb{F}_{p}$, for any positive integer $n$ with respect to its positive divisors.

Corollary 2.1.12. Let $\mathbb{F}_{p}$ be the finite field of characteristic $p$, then $\mathbb{F}_{p^{n}}$ is Galois over $\mathbb{F}_{p}$ and $\operatorname{Gal}\left(\mathbb{F}_{p^{n}} / \mathbb{F}_{p}\right)=<\phi_{p}>$, the Frobenius p-automorphism.

Proof. The extension $\mathbb{F}_{p^{n}}$ over $\mathbb{F}_{p}$ is Galois since it is the splitting field of $x^{p^{n}}-x \in$ $\mathbb{F}_{p}^{*}$. The Frobenius map was shown above to be an automorphism, and is of order $n$, so must generate the Galois group of $\mathbb{F}_{p^{n}}$ over $\mathbb{F}_{p}$.

Proposition 2.1.13. Let $q=p^{m}$ for a prime $p$ and let $\mathbb{F}_{q}=\mathbb{F}_{p^{m}}$ be the finite field with $q$ elements. Let $\phi_{q}=\phi_{p}{ }^{m}$ where $\phi_{p}$ is the Frobenius automorphism. Then any extension of $\mathbb{F}_{q}$ of degree $n$ is the splitting field of $x^{q^{n}}-x$ over $\mathbb{F}_{q}$, and therefore unique and Galois. Furthermore, $\phi_{q}$ generates the Galois group of $\mathbb{F}_{q^{n}}$ over $\mathbb{F}_{q}$. Finally, the subfields of $\mathbb{F}_{q^{n}}$ over $\mathbb{F}_{q}$ are in bijective correspondence with the divisors of $n$.

Proof. We first show that $\phi_{q}$ fixes $\mathbb{F}_{q}$. Let $\alpha \in \mathbb{F}_{q}$, then $\phi_{q}(\alpha)=\phi_{p}{ }^{m}(\alpha)=$ $\phi_{p}(\alpha)^{m}=\alpha^{p^{m}}=\alpha$ since $\mathbb{F}_{q}$ is the splitting field of $x^{p^{m}}-x$ over $\mathbb{F}_{p}$.

Let $\mathbb{F}_{q^{n}}$ be a finite extension of $\mathbb{F}_{q}$ of degree $n$. Then $\mathbb{F}_{q^{n}}$ is the finite extension of $\mathbb{F}_{p}$ of degree $m n$, and is the splitting field of $x^{p^{m n}}-x$ over $\mathbb{F}_{p}$ and therefore unique and Galois. It is an easy exercise, therefore, to show that $\phi_{q}$ is an automorphism of degree $n$ in $\mathbb{F}_{q^{n}}$ and we assert that $\phi_{q}$ generates the Galois group of $\mathbb{F}_{q^{n}}$ over $\mathbb{F}_{q}$. 
The subfield criterion is a direct result of the Fundamental Theorem of Galois Theory and Lagrange's theorem on the (cyclic) Galois group of $\mathbb{F}_{q^{n}}$ over $\mathbb{F}_{q}$ of order $n$.

Definition 2.1.14. Let $K$ be a Galois extension of $F$. If $\alpha \in K$ the elements $\sigma(\alpha)$ for $\sigma \in G a l(K / F)$ are called the Galois conjugates (or simply conjugates) of $\alpha$ over $F$.

Fact 2.1.15. The Galois conjugates of $\alpha \in \mathbb{F}_{q}$ are $\alpha, \alpha^{q}, \alpha^{q^{2}}, \ldots, \alpha^{q^{n-1}}$.

\subsubsection{Traces and Bases of Finite Fields}

We have derived the existence and uniqueness of finite fields using some elementary field extension theory. This provides motivation for the definitions of some key functions we need for the later chapters.

Definition 2.1.16. Let $K$ be a Galois extension of $F$, and let $\alpha \in K$. Define the trace of $\alpha \in K$ over $F$ by

$$
\operatorname{Tr}_{K / F}(\alpha)=\sum_{\sigma \in \operatorname{Gal}(K / F)} \sigma(\alpha)
$$

Similarly the norm of $\alpha \in K$ over $F$ is defined by

$$
N_{K / F}(\alpha)=\prod_{\sigma \in \operatorname{Gal}(K / F)} \sigma(\alpha)
$$


When the context is clear, we drop the subscripts denoting the extension and base fields.

Fact 2.1.17. Let $\alpha \in \mathbb{F}_{q^{n}}$ over $\mathbb{F}_{q}$. Then the trace of $\alpha$ is given by

$$
\operatorname{Tr}(\alpha)=\alpha+\alpha^{q}+\cdots+\alpha^{q^{n-1}}
$$

Similarly, the norm of $\alpha$ is given by

$$
N(\alpha)=\alpha \alpha^{q} \cdots \alpha^{q^{n-1}}
$$

Now we give some properties of the trace function, and note that analogous results can be obtained for the norm, for example see $[6,25]$.

Proposition 2.1.18. [25] Let $K$ be a Galois extension of a field $F$ and let $\alpha, \beta \in K$. Then $\operatorname{Tr}_{K / F}(\alpha)$ is a linear projection from $K$ to $F$. If $K=\mathbb{F}_{q^{n}}$ and $F=\mathbb{F}_{q}$ then also

1. $\operatorname{Tr}_{K / F}(a)=$ na for all $a \in F$;

2. $\operatorname{Tr}_{K / F}\left(\alpha^{q}\right)=\operatorname{Tr}_{K / F}(\alpha)$ for all $\alpha \in K$.

Proof. Let $\alpha \in K, \sigma \in \operatorname{Gal}(K / F)$. Then

$$
\sigma(\operatorname{Tr}(\alpha))=\sigma\left(\sum_{\gamma \in \operatorname{Gal}_{(K / F)}} \gamma(\alpha)\right)
$$


and $\sigma$ is a homomorphism of the additive group of $K$, so

$$
\sigma(\operatorname{Tr}(\alpha))=\sum_{\gamma \in \operatorname{Gal}_{(K / F)}} \sigma \gamma(\alpha)
$$

Left action by $\sigma$ is transitive on $\operatorname{Gal}(K / F)$, so $\sigma(\operatorname{Tr}(\alpha))=\operatorname{Tr}(\alpha)$ and therefore $\operatorname{Tr}(\alpha) \in$ $F$.

Let $\beta \in K$ as well, then

$$
\operatorname{Tr}(\alpha+\beta)=\sum_{\gamma \in \operatorname{Gal}_{(K / F)}} \gamma(\alpha+\beta)=\sum_{\gamma \in \operatorname{Gal}_{(K / F)}} \gamma \alpha+\sum_{\gamma \in \operatorname{Gal}_{(K / F)}} \gamma \beta
$$

since $\gamma$ is a homomorphism of the additive group of $K$. Also, for any $c \in F$

$$
\operatorname{Tr}(c \alpha)=\sum_{\gamma \in \operatorname{Gal}(K / F)} \gamma(c \alpha)
$$

with $\gamma$ a homomorphism of the multiplicative group of $K$ which fixes $F$. Composing these two facts shows the linearity of the trace function.

Now let $K=\mathbb{F}_{q^{n}}$ and $F=\mathbb{F}_{q}$, let $a \in \mathbb{F}_{q}$ and compute

$$
\operatorname{Tr}_{q^{n} / q}(a)=\sum_{i=0}^{n-1} a^{q^{i}}=n a
$$

since $a^{q}=a$. Similarly let $\alpha \in \mathbb{F}_{q^{n}}$, then

$$
\operatorname{Tr}_{q^{n} / q}\left(\alpha^{q}\right)=\sum_{i=0}^{n-1} \alpha^{q^{i+1}}=\operatorname{Tr}_{q^{n} / q}(\alpha)
$$


since $\alpha^{q^{n}}=\alpha$.

The trace function is especially important as it serves as a representation of all linear transformations from $K$ onto $F$ independently of the chosen basis.

Theorem 2.1.19. [25] Consider $\mathbb{F}_{q^{n}}$ as a vector space over $\mathbb{F}_{q}$. Then the linear transformations from $\mathbb{F}_{q^{n}}$ to $\mathbb{F}_{q}$ are precisely the mappings $L_{\beta}$ where $\beta \in \mathbb{F}_{q^{n}}$ such that $L_{\beta}(\alpha)=\operatorname{Tr}(\beta \alpha)$ for all $\alpha \in \mathbb{F}_{q^{n}}$. Furthermore $L_{\beta} \neq L_{\gamma}$ whenever $\beta \neq \gamma$.

Proof. We have shown that each $L_{\beta}$ is a linear transformation from $\mathbb{F}_{q^{n}}$ to $\mathbb{F}_{q}$. For $\beta \neq \gamma$ we have $L_{\beta}(\alpha)-L_{\gamma}(\alpha)=\operatorname{Tr}(\beta \alpha)-\operatorname{Tr}(\gamma \alpha)=\operatorname{Tr}((\beta-\gamma) \alpha)$ which is nonzero for some $\alpha \in \mathbb{F}_{q^{n}}$ as the trace function is an onto mapping. This proves the final assertion and therefore there are $q^{n}$ different mappings $L_{\beta}$ from $\mathbb{F}_{q^{n}}$ to $\mathbb{F}_{q}$. But each mapping onto $\mathbb{F}_{q}$ can be obtained by assigning arbitrary elements of $\mathbb{F}_{q}$ to the $n$ basis elements of $\mathbb{F}_{q^{n}}$ over $\mathbb{F}_{q}$ for any basis. There are $q^{n}$ ways of doing this, exhausting all possible linear transformations.

The following is a simple yet important rule when dealing with compositions of field extensions.

Theorem 2.1.20. (Transitivity of Trace) [25] Let $F=\mathbb{F}_{q}$, let $K$ be an extension of $F$ of degree $m$ and let $E$ be an extension of $K$ of degree $n$. Then for all $\alpha \in E$,

$$
\operatorname{Tr}_{E / F}(\alpha)=\operatorname{Tr}_{K / F}\left(\operatorname{Tr}_{E / K}(\alpha)\right)
$$


Proof. Define $F, K, E$ as in the hypothesis, then

$$
\begin{aligned}
\operatorname{Tr}_{K / F}\left(\operatorname{Tr}_{E / K}(\alpha)\right. & =\sum_{i=0}^{m-1} \operatorname{Tr}_{E / K}(\alpha)^{q^{i}}=\sum_{i=0}^{m-1} \sum_{j=0}^{n-1} \alpha^{q^{j m+i}} \\
& =\sum_{k=0}^{m n-1} \alpha^{q^{k}} \\
& =\operatorname{Tr}_{E / F}(\alpha) .
\end{aligned}
$$

Finally, we use the notion of the trace function to define a property which allows us to easily check linear independence of a group of elements.

Definition 2.1.21. [10] Let $F=\mathbb{F}_{q}$ and $K=\mathbb{F}_{q^{n}}$, let $\alpha_{1}, \alpha_{2}, \ldots, \alpha_{n} \in K$. Then the discriminant of $\alpha_{1}, \alpha_{2}, \ldots, \alpha_{n}$, denoted $\Delta_{K / F}\left(\alpha_{1}, \ldots, \alpha_{n}\right)$ is given by

$$
\Delta_{K / F}\left(\alpha_{1}, \ldots, \alpha_{n}\right)=\left|\begin{array}{cccc}
\operatorname{Tr}_{K / F}\left(\alpha_{1} \alpha_{1}\right) & \operatorname{Tr}_{K / F}\left(\alpha_{1} \alpha_{2}\right) & \ldots & \operatorname{Tr}_{K / F}\left(\alpha_{1} \alpha_{n}\right) \\
\operatorname{Tr}_{K / F}\left(\alpha_{2} \alpha_{1}\right) & \operatorname{Tr}_{K / F}\left(\alpha_{2} \alpha_{2}\right) & \ldots & \operatorname{Tr}_{K / F}\left(\alpha_{2} \alpha_{n}\right) \\
\vdots & \vdots & & \vdots \\
\operatorname{Tr}_{K / F}\left(\alpha_{n} \alpha_{1}\right) & \operatorname{Tr}_{K / F}\left(\alpha_{n} \alpha_{2}\right) & \ldots & \operatorname{Tr}_{K / F}\left(\alpha_{n} \alpha_{n}\right)
\end{array}\right|
$$

The discriminant is an $F$-linear combination of elements in $F$, and therefore $\Delta_{K / F}\left(\alpha_{1}, \alpha_{2}, \ldots, \alpha_{n}\right) \in F$

Theorem 2.1.22. [10] Let $N=\left\{\alpha_{1}, \alpha_{2}, \ldots, \alpha_{n}\right\}$ be any $n$ elements in $\mathbb{F}_{q^{n}}$, then $N$ is a basis of $\mathbb{F}_{q^{n}}$ over $\mathbb{F}_{q}$ if and only if $\Delta_{\mathbb{F}_{q^{n}} / \mathbb{F}_{q}}(N) \neq 0$. 
Proof. Suppose $N=\left\{\alpha_{1}, \alpha_{2}, \ldots, \alpha_{n}\right\}$ forms a basis of $\mathbb{F}_{q^{n}}$ over $\mathbb{F}_{q}$. Let $\beta=$ $c_{1} \alpha_{1}+c_{2} \alpha_{2}+\cdots+c_{n} \alpha_{n}$ for $c_{1}, c_{2}, \ldots, c_{n} \in \mathbb{F}_{q}$, and suppose that $\operatorname{Tr}\left(\beta \alpha_{j}\right)=0$ for $1 \leq j \leq n$. For any $\alpha \in \mathbb{F}_{q^{n}}, \alpha=d_{1} \alpha_{1}+d_{2} \alpha_{2}+\cdots+d_{n} \alpha_{n}$, so

$$
\operatorname{Tr}(\beta \alpha)=d_{1} \operatorname{Tr}\left(\beta \alpha_{1}\right)+d_{2} \operatorname{Tr}\left(\beta \alpha_{2}\right)+\cdots+d_{n} \operatorname{Tr}\left(\beta \alpha_{n}\right)=0
$$

This can only occur for all $\alpha \in \mathbb{F}_{q^{n}}$ if $\beta=0$ and since $\left\{\alpha_{1}, \alpha_{2}, \ldots, \alpha_{n}\right\}$ form a basis for $\mathbb{F}_{q^{n}}$ over $\mathbb{F}_{q}$ we have $c_{1}=c_{2}=\cdots=c_{n}=0$. Therefore the rows of the matrix in Equation (2.1) are linearly independent and so $\Delta(N) \neq 0$.

Suppose conversely $\Delta(N) \neq 0$ and let $c_{1} \alpha_{1}+c_{2} \alpha_{2}+\cdots+c_{n} \alpha_{n}=0$ for some $c_{1}, c_{2}, \ldots, c_{n} \in \mathbb{F}_{q}$. Then

$$
c_{1} \alpha_{1} \alpha_{j}+c_{2} \alpha_{2} \alpha_{j}+\cdots+c_{n} \alpha_{n} \alpha_{j}=0
$$

for $1 \leq j \leq n$ and taking the trace we find

$$
c_{1} \operatorname{Tr}\left(\alpha_{1} \alpha_{j}\right)+c_{2} \operatorname{Tr}\left(\alpha_{2} \alpha_{j}\right)+\cdots+c_{n} \operatorname{Tr}\left(\alpha_{n} \alpha_{j}\right)=0
$$

Since $\Delta(N) \neq 0$, the rows of the matrix in Equation (2.1) are linearly independent, giving $c_{1}=c_{2}=\cdots=c_{n}=0$. 
Corollary 2.1.23. Let $N=\left\{\alpha_{1}, \alpha_{2}, \ldots, \alpha_{n}\right\} \subseteq \mathbb{F}_{q^{n}}$ and let

$$
A=\left(\begin{array}{cccc}
\alpha_{1} & \alpha_{2} & \cdots & \alpha_{n} \\
\alpha_{1}^{q} & \alpha_{2}^{q} & \cdots & \alpha_{n}^{q} \\
\vdots & \vdots & & \vdots \\
\alpha_{1}^{q^{n-1}} & \alpha_{2}^{q^{n-1}} & \cdots & \alpha_{n}^{q^{n-1}}
\end{array}\right)
$$

then $N$ forms a basis of $\mathbb{F}_{q^{n}}$ over $\mathbb{F}_{q}$ if and only if $\operatorname{det}(A) \neq 0$.

Proof. This follows directly from $\operatorname{det}\left(A^{t} \cdot A\right)=(\operatorname{det}(A))^{2}=\Delta(N)$.

\subsection{Introduction to Normal Bases}

This remainder of the chapter provides an overview of some key definitions and results regarding normal bases. Unless otherwise indicated, all definitions and theorems are from the Ph.D thesis of S. Gao [10].

For the remainder of this work we make the following assumptions: let $p$ be a prime, $q$ a power of $p$. Let $\mathbb{F}_{q}$ be the finite field with $q$ elements, and $\mathbb{F}_{q^{n}}$ the extension of dimension $n$ over $\mathbb{F}_{\boldsymbol{q}}$. We note that $\mathbb{F}_{q^{n}}$ can be viewed as a vector space over $\mathbb{F}_{q}$, and therefore any basis has precisely $n$ elements.

Definition 2.2.1. A normal basis of $\mathbb{F}_{q^{n}}$ over $\mathbb{F}_{q}$ is a basis of the form $B=\left\{\alpha, \alpha^{q}, \ldots\right.$, $\left.\alpha^{q^{n-1}}\right\}$ for some $\alpha \in \mathbb{F}_{q^{n}}$. We say that the element $\alpha$ generates the normal basis $B$ and that $\alpha$ is a normal element. 
We observe that the basis $B$ is the set $\alpha$ together with its (Galois) conjugates. For the sake of simplicity we represent the $i$ th conjugate $\alpha^{q^{i}}=\alpha_{i}$ unless the context requires that we use the original form.

We first begin by giving the classical result that normal bases exist for any finite field. This theorem was first proven by Hensel [19] in 1888, and was later generalized for any finite Galois extension by Noether in 1934. Ore independently proved this result in 1934 using "linearized polynomials"; for a discussion of this method see [25]. First, we introduce a notion from linear algebra necessary to prove the result.

Definition 2.2.2. Let $V$ be an n-dimensional vector space over a field $F$ and let $T$ be a linear transformation $V \rightarrow V$. The minimal polynomial $m$ of $T$ is the (monic) polynomial of least degree such that $m(T)=0$. The characteristic polynomial $f$ of $T$ is the product of $(x-\lambda)$ over all eigenvalues $\lambda$ of $T$.

The Cayley-Hamilton theorem states that the minimal polynomial of $T$ always divides the characteristic polynomial of $T$.

Definition 2.2.3. A cyclic vector $\alpha \in V$ of a linear transformation $T$ is an element such that $\alpha, T(\alpha), T^{2}(\alpha), \ldots, T^{n-1}(\alpha)$ forms a basis for $V$ over $F$.

We give as a lemma a standard result from linear algebra.

Lemma 2.2.4. [20] Let $V$ be an $n$-dimensional vector space over a field $F$ and let $T$ be a linear transformation $V \rightarrow V$. Then $V$ contains a cyclic vector of $T$ if and only if the minimal polynomial of $T$ equals the characteristic polynomial of $T$. 
Proof. Let $m$ be the minimal polynomial of a linear transformation $T$ from $V \rightarrow V$ over $F$, and let $f$ be the characteristic polynomial of $T$. Suppose that $T$ admits a cyclic vector $\alpha \in V$. Then $\alpha, T(\alpha), \ldots, T^{n-1}(\alpha)$ form a basis of $V$ over $F$. That is, $\alpha, T(\alpha), \ldots, T^{n-1}(\alpha)$ are linearly independent over $F$. Suppose the degree of $m$ is less than $n$, say $m(x)=m_{0}+m_{1} x+\cdots+m_{k} x^{k}$ for some $k<n$. Then $m(T)=$ $m_{0} 1+m_{1} T+\cdots+m_{k} T^{k}=0$. In particular,

$$
m_{0} \alpha+m_{1} T(\alpha)+\cdots+m_{k} T^{k}(\alpha)=0
$$

but linear independence of $\alpha, T(\alpha), \ldots, T^{n-1}(\alpha)$ guarantees that $m_{0}=m_{1}=\cdots=$ $m_{k}=0$, contradicting the choice of $m$. Thus, the degree of $m$ is at least $n$, but since $m$ divides $f$ and $f$ is monic of degree $n$, we have $m=f$.

Suppose the converse, that the minimal polynomial of a linear transformation $T: V \rightarrow V$ over $F$ is equal to the characteristic polynomial of $T$. Then the degree of the minimal polynomial of $T$ is $n$. Suppose there is no cyclic vector $\alpha \in V$; that is $\alpha, T(\alpha), \ldots, T^{n-1}(\alpha)$ is linearly dependent for all $\alpha \in V$. Then there is a non-trivial second block in the rational decomposition of $T$. Let

$$
A=\left(\begin{array}{cccc}
M_{1} & 0 & 0 & 0 \\
\dot{0} & M_{2} & 0 & 0 \\
\vdots & & \ddots & \vdots \\
0 & 0 & 0 & M_{k}
\end{array}\right)
$$


be the rational decomposition of $T$, where $M_{2}$ is the companion matrix to a polynomial $p$, and $p$ divides $m$. By the assumption, $M_{2}$ is non-trivial so $p$ is non-constant. The matrix

$$
B=\left(\begin{array}{cccc}
0 & 0 & 0 & 0 \\
0 & I & 0 & 0 \\
\vdots & & \ddots & \vdots \\
0 & 0 & 0 & I
\end{array}\right)
$$

commutes with $A$. We show an equivalent form of the theorem: that $B$ is not a polynomial of degree less than $n$. If $B=f(A)$ for some polynomial $f$, then $f\left(M_{1}\right)=0$ and therefore $m$ divides $f$. We have that $p$ divides $m$ and so $f\left(M_{2}\right)=0$, which contradicts construction of $B$.

Theorem 2.2.5. (Normal basis theorem for finite fields) Every extension $\mathbb{F}_{q^{n}}$ over $\mathbb{F}_{q}$ has a normal basis.

Proof. We have shown that the Galois group of $\mathbb{F}_{q^{n}}$ over $\mathbb{F}_{q}$ is generated by the Frobenius $q$-automorphism $\phi_{q}$. Since $\phi_{q}$ is a field automorphism that fixes $\mathbb{F}_{q}$, we have that $\phi_{q}(a+b)=\phi_{q}(a)+\phi_{q}(b)$ and $\phi_{q}(c a)=\phi_{q}(c) \phi_{q}(a)=c \phi_{q}(a)$ for all $a, b \in \mathbb{F}_{q^{n}}, c \in \mathbb{F}_{q}$. In other words, we can consider $\phi_{q}$ as a linear operator on the $n$-dimensional vector space $\mathbb{F}_{q^{n}}$ over $\mathbb{F}_{q}$.

Since $\phi_{q}^{n}=$ id, the polynomial $x^{n}-1 \in \mathbb{F}_{q}[x]$ annihilates $\phi_{q}$. Since the first $n-1$ powers of $\phi_{q}$ are linearly independent, no polynomial of degree less than $n$ can annihilate $\phi_{q}$. It follows that both the characteristic polynomial and minimal polynomial of 
$\phi_{q}$ are $x^{n}-1$. By Lemma 2.2 .4 there exists an $\alpha \in \mathbb{F}_{q^{n}}$ such that $\left\{\alpha, \phi_{q}(\alpha), \phi_{q}^{2}(\alpha), \ldots\right\}$ spans $\mathbb{F}_{q^{n}}$. Since $x^{n}-1$ annihilates $\phi_{q}$ we have $\left\{\alpha, \phi_{q}(\alpha), \ldots, \phi_{q}^{n-1}(\alpha)\right\}$ spans $\mathbb{F}_{q^{n}}$. Thus, the element $\alpha$ and its conjugates form a basis for $\mathbb{F}_{q^{n}}$ over $\mathbb{F}_{q}$.

Viewing $\mathbb{F}_{q^{n}}$ as an $n$-dimensional vector space over $\mathbb{F}_{q}$, we can identify any element of $\mathbb{F}_{q^{n}}$ as an $n$-tuple with respect to a basis $\left\{\alpha_{0}, \alpha_{1}, \ldots, \alpha_{n-1}\right\}$ over $\mathbb{F}_{q}$. For any $\alpha, \beta \in \mathbb{F}_{q^{n}}$ we have $\alpha=a_{0} \alpha_{0}+a_{1} \alpha_{1}+\cdots+a_{n-1} \alpha_{n-1}$ and $\beta=b_{0} \alpha_{0}+b_{1} \alpha_{1}+\cdots+b_{n-1} \alpha_{n-1}$ for $a_{i}, b_{i} \in \mathbb{F}_{q} ; \quad 0 \leq i \leq n-1$.

\subsubsection{Arithmetic Using Normal Bases}

We identify an arbitrary $\alpha \in \mathbb{F}_{q^{n}}$ with the vector $A=\left(a_{0}, a_{1}, \ldots, a_{n-1}\right)$ and $\beta \in \mathbb{F}_{q^{n}}$ with $B=\left(b_{0}, b_{1}, \ldots, b_{n-1}\right)$. Then addition of $\alpha$ and $\beta$ is component-wise, i.e:

$$
A+B=\left(a_{0}+b_{0}, a_{1}+b_{1}, \ldots, a_{n-1}+b_{n-1}\right) .
$$

If our basis is a normal basis consider the vector $A^{q}=\left(a_{n-1}, a_{0}, a_{1}, \ldots, a_{n-2}\right)$; taking $q$ th powers is simply a cyclic bit-shift of the coefficients, and is cost-negligible. For this reason normal bases are highly used in all applications that require taking large powers, particularly in public-key cryptography.

Multiplication requires more computation. If $A B=C=\left(c_{0}, c_{1}, \ldots, c_{n-1}\right)$ then the goal is to represent the $c_{i}$ coefficients in terms of the $a_{i}$ and $b_{i}$ coefficients. We 
begin by defining the $n \times n$ matrix $T_{k}=\left(t_{i j}^{(k)}\right)$ by

$$
\alpha_{i} \alpha_{j}=\sum_{k=0}^{n-1} t_{i j}^{(k)} \alpha_{k} \quad t_{i j}^{(k)} \in \mathbb{F}_{\boldsymbol{q}}
$$

Then,

$$
\begin{aligned}
\alpha \beta & =\left(a_{0} \alpha_{0}+a_{1} \alpha_{1}+\cdots+a_{n-1} \alpha_{n-1}\right)\left(b_{0} \alpha_{0}+b_{1} \alpha_{1}+\cdots+b_{n-1} \alpha_{n-1}\right) \\
& =\sum_{i=0}^{n-1} \sum_{j=0}^{n-1} a_{i} \alpha_{i} b_{j} \alpha_{j} \\
& =\sum_{k=0}^{n-1} \sum_{i, j=0}^{n-1} a_{i} b_{j} t_{i j}^{(k)} \alpha_{k}
\end{aligned}
$$

and the $k$ th coefficient of $C$ is given by

$$
c_{k}=\sum_{i, j=0}^{n-1} a_{i} b_{j} t_{i j}^{(k)}=A T_{k} B^{t}, \quad 0 \leq k \leq n-1
$$

The problem of multiplication reduces to finding the collection of matrices $T_{k}$.

Definition 2.2.6. The multiplication table of a basis $\left\{\alpha_{0}, \alpha_{1}, \ldots, \alpha_{n-1}\right\}$ of $\mathbb{F}_{q^{n}}$ over $\mathbb{F}_{q}$ is the collection of $n$ matrices $T_{k}$ as outlined above.

As the dimension $n$ of the extension increases we notice that the computations required to find the $T_{k}$ matrices become prohibitive. For this reason we must find bases where we can take advantage of symmetry of the $T_{k}$ matrices.

Consider our basis to be a normal basis. Then we can take advantage of the exponentiation property in the following way. We take the $q^{-l}$ th power of Equation (2.3) 
to find the identity

$$
\alpha_{i-l} \alpha_{j-l}=\sum_{k=0}^{n-1} t_{i j}^{(k)} \alpha_{k-l}
$$

Matching up the coefficient of $\alpha_{0}$ in Equations (2.3) and (2.4) gives the identity

$$
t_{i j}^{(l)}=t_{i-l, j-l}^{(0)}
$$

Therefore we find that once a computation scheme is built to calculate the first coefficient $c_{0}$, we can simply run the system using $A^{q^{-l}}$ and $B^{q^{-l} t}$ with negligible computation cost as inputs to find $c_{l}, 1 \leq l \leq n-1$. The number of operations required in any such computational scheme is the number of non-zero entries in the matrix $T_{0}$.

Again, let $N=\left\{\alpha, \alpha_{1}, \ldots, \alpha_{n-1}\right\}$ be a normal basis of $\mathbb{F}_{q^{n}}$ over $\mathbb{F}_{q}$. We begin by defining the $n \times n$ matrix $T=\left(t_{i j}\right)$ by the following system

$$
\alpha \alpha_{i}=\sum_{j=0}^{n-1} t_{i j} \alpha_{j} \quad 0 \leq i \leq n-1
$$

Recall, for any $i, j, k \leq n-1$ we have

$$
\alpha \alpha_{i-j}=\sum_{l=0}^{n-1} t_{i-j, l} \alpha_{l}
$$


Taking both sides to the $q^{j}$ th power we find,

$$
\alpha_{j} \alpha_{i}=\sum_{l=0}^{n-1} t_{i-j, l} \alpha_{l+j}
$$

Reindexing with $k=l+j$ gives that for any $i, j, k \leq n-1$

$$
t_{i j}^{(k)}=t_{i-j, k-j}
$$

In particular, this shows that the number of non-zero entries in the matrix $T_{0}$ is equal to the number of non-zero entries in $T$. Moreover, the collection of matrices $\left\{T_{k}\right\}$ are completely determined by the matrix $T$.

Definition 2.2.7. If $N=\left\{\alpha_{0}, \alpha_{1}, \ldots, \alpha_{n-1}\right\}$ is a normal basis, the matrix $T=\left(t_{i j}\right)$ defined as in Equation (2.5) is called the multiplication table of $N$.

Definition 2.2.8. Let $T$ be the multiplication table of a normal basis $N$. Then the complexity of $T$ is the number of non-zero entries of $T$. The complexity of the normal basis $N$ is denoted $c_{N}$. Also, if $\alpha$ generates a normal basis, i.e.: $\alpha$ is a normal element, then the complexity of $\alpha$ is the number of non-zero entries in the multiplication table of the basis generated by $\alpha$. 


\subsection{Optimal Normal Bases}

The following theorem is due to Mullin et al., and gives a very important lower bound on the complexity of normal bases.

Theorem 2.3.1. [31] Let $N$ be a normal basis of $\mathbb{F}_{q^{n}}$ over $\mathbb{F}_{q}$, then $c_{N} \geq 2 n-1$.

Proof. Let $N$ be a normal basis of $\mathbb{F}_{q^{n}}$ over $\mathbb{F}_{q}$ generated by $\alpha$ with multiplication table $T$. Let

$$
\begin{aligned}
a=\operatorname{Tr}(\alpha) & =\sum_{i=0}^{n-1} \alpha_{i} \text { then } \\
a \cdot \alpha & =\sum_{i=0}^{n-1} \alpha \alpha_{i}
\end{aligned}
$$

so that the sum of the rows of $T$ is an $n$-tuple with an $a$ in the first position and 0 elsewhere. Since the rows of $T$ are linearly independent, there must be at least two non-zero terms in each column of $T$ except for the first column. Thus, the total number of non-zero entries of $T$ is at least $2 n-1$.

Definition 2.3.2. Consider $\mathbb{F}_{q^{n}}$ over $\mathbb{F}_{q}$. The complexity of $\mathbb{F}_{q^{n}}$ over $\mathbb{F}_{q}$, denoted $C_{q}(n)$, is the minimum complexity of a normal basis in $\mathbb{F}_{q^{n}}$.

Definition 2.3.3. Let $N$ be a normal basis of $\mathbb{F}_{q^{n}}$ over $\mathbb{F}_{q}$ then $N$ is an Optimal Normal Basis $(O N B)$ if $c_{N}=2 n-1$.

Two constructions for optimal normal bases were given by Mullin et al., and it was conjectured that all optimal normal bases were given by these constructions. This 
was later proven by Gao [10].

\subsubsection{Characterization of Optimal Normal Bases}

We begin by giving the constructions shown by Mullin et al.

Theorem 2.3.4. [31] Let $n+1$ be a prime in $\mathbb{Z}$ and suppose $q$ is a prime or a prime power such that $q$ is primitive in $\mathbb{Z}_{n+1}$. Then the $n$ non-unit $(n+1)$ th primitive roots of unity form an optimal normal basis of $\mathbb{F}_{q^{n}}$ over $\mathbb{F}_{q}$.

Proof. We have $(n+1)$ is a prime so $\mathbb{Z}_{n+1}$ is a field. If $q$ is primitive in $\mathbb{Z}_{n+1}$ then $q$ generates the multiplicative group of $\mathbb{Z}_{n+1}$ and so $q^{n} \equiv 1(\bmod n+1)$. Therefore $n+1$ divides $q^{n}-1$. Let $\eta$ be primitive element of $\mathbb{F}_{q^{n}}$, then let $\beta=\eta^{q^{n}-1 /(n+1)}$ so that $\beta$ is a primitive $(n+1)$ th root of unity and all the conjugates $\beta, \beta^{q}, \ldots, \beta^{q-1}$ are primitive $(n+1)$ th roots of unity.

Since $q$ is primitive in $\mathbb{Z}_{n+1}$ the minimal polynomial of $\beta$ is $\left(x^{n+1}-1\right) /(x-1)$, with the conjugates as the (linearly independent) roots. This shows that the primitive $(n+1)$ th roots of unity form a normal basis of $\mathbb{F}_{q^{n}}$ over $\mathbb{F}_{q}$.

Let $\beta_{i}=\beta^{q^{i}}$ in the usual convention. If $\beta_{i} \neq \beta^{-1}$ then $\beta \beta_{i}=\beta_{j}$ for some $1 \leq j \neq i \leq n-1$. If $\beta_{i}=\beta^{-1}$ then $\beta \beta_{i}=1=-\operatorname{Tr}(\beta)$ and so row $i$ of the multiplication table is $(-1,-1, \ldots,-1)$. In total, the multiplication table of this basis has $(n-1)+n=2 n-1$ non-zero terms, and therefore is optimal.

Theorem 2.3.5. [31] Let $2 n+1$ be prime and suppose that 
(1) 2 is primitive in $\mathbb{Z}_{2 n+1}$, or

(2) $2 n+1 \equiv 3(\bmod 4)$ and 2 generates the quadratic residues in $\mathbb{Z}_{2 n+1}$.

Then $\beta=\gamma+\gamma^{-1}$ generates an optimal normal basis of $\mathbb{F}_{2^{n}}$ over $\mathbb{F}_{2}$, where $\gamma$ is a primitive $2 n+1$ root of unity.

Proof. We begin by restating condition $(2):(2 n+1) \equiv 3(\bmod 4)$ implies that $(2 n+1)$ cannot be written as the sum of two squares. If $a^{2} \equiv-1(\bmod 2 n+1)$ for some $a \in \mathbb{Z}_{2 n+1}$. Then $a^{(2 n+1)-1}=(-1)^{(2 n) / 2} \equiv-1(\bmod 2 n+1)$. But, by Fermat's little theorem $a^{2 n} \equiv 1(\bmod 2 n+1)$. Thus, $(-1)$ is not a quadratic residue $(\bmod 2 n+1)$. Also, 2 generates the quadratic residues in $\mathbb{Z}_{2 n+1}$ is equivalent to saying that the multiplicative order of $2(\bmod 2 n+1)$ is $n$. Thus, $\mathbb{Z}_{2 n+1}$ is generated by 2 and -1 .

If the first condition holds, then $2^{2 n} \equiv 1(\bmod 2 n+1)$ so $2^{2 n}-1 \equiv 0(\bmod 2 n+1)$, or $2 n+1$ divides $2^{2 n}-1$. If the second condition holds then $2^{n}=2^{2 n} \equiv 1(\bmod 2 n+1)$ and so also $2 n+1$ divides $2^{2 n}-1$.

So, as in the proof of Theorem 2.3.4, in $\mathbb{F}_{2^{2 n}}$ there is an element $\gamma$ that is a primitive $(2 n+1)$ th root of unity in $\mathbb{F}_{2^{2 n}}$. We note that $\left(2^{n}\right)^{2}=2^{2 n} \equiv 1(\bmod 2 n+1)$ so $2^{n} \equiv \pm 1(\bmod 2 n+1)$. Letting $\beta=\gamma+\gamma^{-1}$ we note that $\gamma^{2^{n}}=\gamma$ or $\gamma^{2^{n}}=\gamma^{-1}$. In either case, we have that $\beta^{2^{n}}=\beta$ so $\beta \in \mathbb{F}_{2^{n}} \subseteq \mathbb{F}_{2^{2 n}}$.

To prove that $N=\left\{\beta_{0}, \beta_{1}, \ldots, \beta_{n-1}\right\}$ is linearly independent, suppose $\sum_{i=0}^{n-1} c_{i} \beta_{i}=0$. Then,

$$
\sum_{i=0}^{n-1} c_{i}\left(\gamma^{2^{i}}+\gamma^{-2^{i}}\right)=\sum_{i=0}^{n-1} c_{i} \gamma^{2^{i}}+\sum_{i=0}^{n-1} c_{i} \gamma^{-2^{i}}=0
$$


We recall that $\gamma \in \mathbb{F}_{2^{2 n}}$, and 2 generates the multiplicative group of $\mathbb{Z}_{2 n+1}$ or 2 generates the quadratic residues in $\mathbb{Z}_{2 n+1}$ with $2 n+1 \equiv 3(\bmod 4)$. In either case the exponents $2^{i}$ and $-2^{i}$ run through all non-zero residues $j(\bmod 2 n+1)$ so we have,

$$
\sum_{j=1}^{2 n} \delta_{j} \gamma^{j}=0
$$

and each $c_{i} \in\left\{\delta_{1}, \delta_{2}, \ldots, \delta_{2 n}\right\}$.

Let $f(x)=\sum_{i=0}^{2 n-1} \delta_{i+1} x^{i}$, then $\gamma$ is a root of $f$ and the minimal polynomial of $\gamma$ (the $(2 n+1)$ th cyclotomic polynomial over $\left.\mathbb{Z}_{2 n+1}\right)$, divides $f$. If condition (1) holds, then $m_{\gamma}(x)=1+x+x^{2}+\cdots+x^{2 n}$. Since $m_{\gamma}$ divides $f$ we have $f=0$ and thus all $c_{i}=0$. If condition (2) holds, then the degree of both $m_{\gamma}(x)$ and $m_{\gamma^{-1}}(x)$ is $n$. This gives that

$$
x^{2 n}-1=(x-1) m_{\gamma}(x) m_{\gamma^{-1}}(x)
$$

but $\gamma$ and $\gamma^{-1}$ are roots of $f$, so $m_{\gamma}(x) m_{\gamma^{-1}(x)}=1+x+x^{2}+\cdots+x^{2 n}$ divides $f$, once again giving $f=0$ and all $c_{i}=0$. This shows linear independence of $N=\left\{\beta_{0}, \beta_{1}, \ldots, \beta_{n-1}\right\}$

Let $N=\left\{\beta_{0}, \beta_{1}, \ldots, \beta_{n-1}\right\}$ be the normal basis as above with $\beta=\gamma+\gamma^{-1}$. Then $\beta_{i}=\gamma^{2^{i}}+\gamma^{-2^{i}}=\gamma^{j}+\gamma^{-j}$ for some $1 \leq j \leq n-1$. First, we note that $\beta \beta=\beta^{2} \in N$. 
Now

$$
\begin{aligned}
\beta \beta_{i} & =\left(\gamma+\gamma^{-1}\right)\left(\gamma^{j}+\gamma^{-j}\right) \\
& =\gamma^{j+1}+\gamma^{-(j+1)}+\gamma^{j-1}+\gamma^{-(j-1)} \\
& =\beta_{k}+\beta_{l} \quad \text { for } 0 \leq k, l \leq n-1
\end{aligned}
$$

To conclude, we note that the first row of the multiplication table has a 1 in the first row, and two $1 \mathrm{~s}$ in every subsequent row, for a total complexity $c_{N}=2 n-1$.

Definition 2.3.6. Let $N$ be the optimal normal basis as described in Theorem 2.3.4, then $N$ is called a Type I Optimal Normal Basis.

Let $N$ be the optimal normal basis as described in Theorem 2.3.5, then $N$ is called a Type II Optimal Normal Basis.

By analyzing the construction of some optimal normal bases, we have a quick method for determining some other extension fields with optimal normal bases.

Proposition 2.3.7. Let $\mathbb{F}_{2^{\text {n }}}$ be an extension of $\mathbb{F}_{2}$ containing a Type I optimal normal basis with $n$ even. Then, $\mathbb{F}_{2^{n / 2}}$ contains a Type II optimal normal basis.

Proof. Let $n$ be an even positive integer, and let $m=n / 2$. If $\mathbb{F}_{2^{n}}$ contains a Type I Optimal normal basis then $n+1$ is prime and 2 is primitive modulo $(n+1)$. This implies that $2 m+1$ is prime, and 2 is primitive modulo $(2 m+1)$, or that $\mathbb{F}_{2^{m}}$ contains a Type II optimal normal basis. 
We end this part by giving an equivalence class on normal bases.

Definition 2.3.8. Suppose $N=\left\{\beta_{0}, \beta_{1}, \ldots, \beta_{n-1}\right\}$ is a normal basis of $\mathbb{F}_{q^{n}}$ over $\mathbb{F}_{q}$. Then, for any nonzero $a \in \mathbb{F}_{q}, a N=\left\{a \beta_{i}: 0 \leq i \leq n-1\right\}$ is an equivalent basis of $N$

We are concerned with the property that equivalent bases preserve complexity. We show this in the case of optimal normal bases but note that the proof holds for any normal basis.

Proposition 2.3.9. Suppose $N=\left\{\beta_{0}, \beta_{1}, \ldots, \beta_{n-1}\right\}$ is an optimal normal basis of $\mathbb{F}_{q^{n}}$ over $\mathbb{F}_{q}$. Then the equivalent basis aN $=\left\{a \beta_{i}: 0 \leq i \leq n-1\right\}$ is an optimal normal basis of $\mathbb{F}_{q^{n}}$ over $\mathbb{F}_{q}$ for any non-zero $a \in \mathbb{F}_{q}$.

Proof. It is clear that the linear independence of the elements of $a N$ follows from the linear independence of $N$. Also, for $\beta$ generating $N, a \beta(a \beta)_{i}=a a^{q^{i}} \beta \beta_{i}=a^{2} \beta \beta_{i}$ which indicates that the number of nonzero terms of the multiplication table remains the same.

\subsubsection{Determination of all Optimal Normal Bases}

The natural question to ask is: do any other optimal normal bases exist over finite fields? In 1988, Mullin et al. [31] performed exhaustive computer searches over $\mathbb{F}_{2^{n}}$ for $n \leq 30$, and no new optimal normal bases were found. We present the original proof of Gao: that Theorems 2.3.4, 2.3.5 and their equivalent bases describe all optimal 
normal bases in finite field extensions. Later, Gao and Lenstra [12] generalized the notion of an optimal normal basis to any finite Galois extension of fields.

Theorem 2.3.10. [10] Let $N=\left\{\alpha, \alpha^{q}, \ldots, \alpha^{q^{n-1}}\right\}$ be an optimal normal basis of $\mathbb{F}_{q^{n}}$ over $\mathbb{F}_{q}$ and let $b=\operatorname{Tr}_{q^{n} / q}(\alpha)$. Then either

1. $n+1$ is prime, $q$ primitive in $\mathbb{Z}_{n+1}$ and $-\alpha / b$ is a primitive $(n+1)$ th root of unity; or

2. (a) $q=2^{\nu}$ for some integer $\nu$ such that $\operatorname{gcd}(\nu, n)=1$,

(b) $2 n+1$ is a prime, 2 and -1 generate the multiplicative group $\mathbb{Z}_{2 n+1}^{*}$, and (c) $\alpha / b=\gamma+\gamma^{-1}$ for some primitive $(2 n+1)$ th root of unity $\gamma$.

Remark. We note that this is the sufficiency condition that any optimal normal basis of $\mathbb{F}_{q^{n}}$ over $\mathbb{F}_{q}$ is equivalent to a Type I or Type II optimal normal basis as introduced above. We also note, for the case $q=2$, that this ensures uniqueness of optimal normal bases of $\mathbb{F}_{2^{n}}$ over $\mathbb{F}_{2}$.

Proof. Let $N=\left\{\alpha_{0}, \alpha_{1}, \ldots, \alpha_{n-1}\right\}$ be an optimal normal basis of $\mathbb{F}_{q^{n}}$ over $\mathbb{F}_{q}$ with $n \times n$ multiplication table $T=\left(t_{i j}\right)$ and let $M=\left\{\beta_{0}, \beta_{1}, \ldots, \beta_{n-1}\right\}$ be the dual (normal) basis of $N$ (see Section 2.6) with multiplication table $D=\left(d_{i j}\right)$. By Lemma 2.6.5 we have $D=T^{t}$. Also by Equation (2.6) we have $d_{i j}=d_{i-j,-j}$.

By the proof of Theorem 2.3.1 we have that each column of $D$ has exactly two non-zero entries which are additive inverses except for the first column, which has 
only one non-zero entry with value $b=\operatorname{Tr}(\alpha)$. Therefore

$$
\begin{aligned}
& \alpha \beta_{i}=a \beta_{j}-a \beta_{k} ; \quad a \in \mathbb{F}_{q}, 0 \leq i \neq j \neq k \leq n-1, \text { and } \\
& \alpha \beta_{0}=b \beta_{m} ; \quad \text { for } 0 \leq m \leq n-1 .
\end{aligned}
$$

Replacing $\alpha$ with $\alpha / b$ and $\beta$ with $b \beta$ without loss of generality we may assume that $\operatorname{Tr}(\alpha)=-1$ and $\alpha \beta_{0}=-\beta_{m}$. By the proof of Theorem 2.3.1 we have

$$
\operatorname{Tr}(\alpha) \operatorname{Tr}(\beta)=\sum_{i, j} \alpha_{i} \beta_{j}=\sum_{k=0}^{n-1} \alpha \beta_{k}=1
$$

and so $\operatorname{Tr}(\beta)=-1$.

If $m=0$ then $\alpha \beta_{0}=-\beta_{m}$ and $\alpha=-1$ which gives $n=1$. This covers the trivial case so for the remainder of the proof assume $m>0$.

Suppose $2 m \equiv 0(\bmod n)$. Then

$$
\left(\alpha \beta_{0}\right)^{q^{m}}=\alpha_{m} \beta_{m}=-\beta_{2 m}=-\beta_{0}=\beta_{m} / \alpha
$$

Rearranging gives

$$
\alpha \alpha_{m}=1=-\operatorname{Tr}(\alpha)=\sum_{i=0}^{n-1}-\alpha_{i}
$$

so for each $i \neq 0$, there is a unique $s \neq m$ such that $\alpha \beta_{i}=\beta_{s}-\beta_{m}$. Define

$$
\phi:\{1,2, \ldots, n-1\} \rightarrow\{0,1, \ldots, n-1\}-\{m\}
$$


by $\phi(i)=s$ as above. If $i \neq j$ then $\alpha \beta_{i} \neq \alpha \beta_{j}$; so if $t \neq m$ is the unique integer such that $\alpha \beta_{j}=\beta_{t}-\beta_{m}$ we have $s \neq t$ or $\phi(i) \neq \phi(j)$. This shows that $\phi$ is a bijection. This, along with $\alpha \alpha_{m}=1$ shows that $S=\{1\} \bigcup\left\{\alpha_{0}, \alpha_{1}, \ldots, \alpha_{n-1}\right\}$ is closed under multiplication by $\alpha$. The set $S$ is also closed under the Frobenius map and we find $S$ is a multiplicative group of order $n+1$. Then $\alpha^{n+1}=1, \alpha \neq 1$ and so $\alpha$ is a root of $x^{n+1}-1 /(x-1)=x^{n}+x^{n-1}+\cdots+x+1$. Since $\alpha$ is of degree $n$ over $\mathbb{F}_{q}$ we have $x^{n}+x^{n-1}+\cdots+x+1$ is irreducible, which occurs if and only if $n+1$ is prime, see [6, Section 13.6]. This means that $N$ is equivalent to a Type I Optimal normal basis.

Now suppose $m \neq 0$ and $2 m \not \equiv 0(\bmod n)$. What remains is to show that $N$ is equivalent to a Type II Optimal normal basis. Recall if $i=0$ then $\alpha \beta_{i}=\alpha \beta_{0}=-\alpha_{m}$ and so $d_{0, m}=-1$ and $d_{0, i}=0$ for all other $i$. Using Equation (2.6) gives us that

$$
d_{-i,-i}= \begin{cases}-1 & \text { if } i=m \\ 0 & \text { if } i \neq m\end{cases}
$$

So $\alpha \beta_{-m}=\beta_{l}-\beta_{-m}$ for some $0 \leq l \leq n-1$.

To show that $\mathbb{F}_{\boldsymbol{q}}$ is of characteristic two, we consider

$$
\alpha_{m}\left(\alpha \beta_{0}\right)=-\alpha_{m} \beta_{m}=-(\alpha \beta)^{q^{m}}=\beta_{2 m}
$$


However, viewing this product another way,

$$
\alpha\left(\alpha_{m} \beta_{0}\right)=\alpha\left(\alpha \beta_{-m}\right)^{q^{m}}=\alpha\left(\beta_{l}-\beta_{-m}\right)^{q^{m}}=\alpha \beta_{l+m}-\alpha \beta_{0}=\alpha \beta_{l+m}+\beta_{m}
$$

Combining these two expressions gives

$$
\alpha \beta_{l+m}=\beta_{2 m}-\beta_{m}
$$

We calculate $\alpha \alpha_{l} \beta_{-m}$ also in two ways. We have that $d_{-m, l}=1=d_{-(l+m),-l}$ and $l+m \neq 0$ so that

$$
\alpha \beta_{-(l+m),-l}=\beta_{-l}-\beta_{j}
$$

for some $j \neq-l,-(l+m)$. We proceed as above by calculating

$$
\begin{aligned}
\alpha_{l}\left(\alpha \beta_{-m}\right) & =\alpha_{l}\left(\beta_{l}-\beta_{-m}\right)=\left(\alpha \beta_{0}-\alpha \beta_{-(l+m)}\right)^{q^{l}} \\
& =\left(-\beta_{m}-\beta_{-l}+\beta_{j}\right)^{q^{l}}=-\beta_{l+m}-\beta_{0}+\beta_{l+j} .
\end{aligned}
$$

We also calculate the product as

$$
\begin{aligned}
\alpha\left(\alpha_{l} \beta_{-m}\right) & =\alpha\left(\alpha \beta_{-(l+m)}\right)^{q^{l}}=\alpha\left(\beta_{-l}-\beta_{j}\right)^{q^{l}} \\
& =\alpha \beta_{0}-\alpha \beta_{l+j}=-\beta_{m}-\alpha \beta_{l+j} .
\end{aligned}
$$


Comparing Equations 2.11 and 2.12 gives the identity

$$
\alpha \beta_{l+j}=\beta_{0}+\beta_{l+m}-\beta_{m}-\beta_{l+j}
$$

We note that $\beta$ generates an optimal normal basis, so $\alpha \beta_{l+j}$ has at most two nonzero terms. Since $l+j \neq 0, \beta_{l+j}$ must equal one of $\beta_{l+m}$ and $-\beta_{m}$.

Suppose then, that $\beta_{l+j}=\beta_{l+m}$ i.e: that $l+j=l+m$. Then $\alpha \beta_{l+j}=\beta_{0}-\beta_{m}$ and also by Equation (2.10) $\alpha \beta_{l+j}=\beta_{2 m}-\beta_{m}$. Combining these give $\beta_{2 m}=\beta_{0}$, and therefore $2 m \equiv 0(\bmod n)$, contradicting the assumption.

In the second case, $\beta_{l+j}=-\beta_{m}$ and since $\beta_{l+j}$ and $\beta_{m}$ are basis elements, $l+j=m$ and so $2=0$. This shows we are in characteristic two, i.e: $q=2^{\nu}$.

By Equation (2.13) we have $\alpha \beta_{m}=\beta_{l+m}+\beta_{0}$. We note that Equations (2.8) and (2.9) reduce to $\alpha \beta_{0}=\beta_{m}$ and $\alpha \beta_{-m}=\beta_{l}+\beta_{-m}$ in the case of characteristic two. Raising Equation (2.9) to the $q^{m}$ th power we find $\alpha_{m} \beta_{0}=\beta_{l+m}+\beta_{0}=\alpha \beta_{m}$. Combining this expression with Equation (2.8) we find $\alpha^{2}=\alpha_{m}$. Inductively, for every non-negative integer $k$, we have $\alpha^{2^{k}}=\alpha^{q^{m k}}$.

We pick $k=n / \operatorname{gcd}(m, n)$ to find $\alpha^{2^{k}}=\alpha$, or $\alpha \in \mathbb{F}_{2^{k}}$ and so of degree at most $k \leq n$ over $\mathbb{F}_{2} \subseteq \mathbb{F}_{q}$. Since $\alpha$ is of degree $n$ over $\mathbb{F}_{q}$ it must have degree at least $n$ over $\mathbb{F}_{2}$ and thus $k=n$. Since $\alpha$ has the same degree over $\mathbb{F}_{2}$ as $\mathbb{F}_{q}$, with $q=2^{\nu}$, we have $\nu$ and $n$ are relatively prime and the conjugates of $\alpha$ over $\mathbb{F}_{q}$ are $\left\{\alpha, \alpha^{2}, \ldots, \alpha^{2^{n-1}}\right\}$. This proves the first condition of the second hypothesis in the statement.

Let $m_{1}$ be a positive integer such that $m m_{1} \equiv 1(\bmod n)$. Since $\alpha_{m} \beta=\alpha \beta_{m}$ we 
have $\alpha \beta^{-1}=\alpha_{m} \beta_{m}^{-1}$, and raising the expression to the $q^{m}$ th power we find $\alpha_{m} \beta_{m}^{-1}=$ $\alpha_{2 m} \beta_{2 m}^{-1}$. Inductively we have

$$
\alpha_{m m_{1}} \beta_{m m_{1}}^{-1}=\alpha \beta^{-1}=\alpha^{q} \beta^{-q}
$$

and so $\alpha \beta^{-1} \in \mathbb{F}_{q}$. We have

$$
\operatorname{Tr}(\alpha)=1=\operatorname{Tr}\left(\alpha \beta^{-1} \beta\right)=\alpha \beta^{-1} \operatorname{Tr}(\beta)
$$

and so $\alpha=\beta$ since $\operatorname{Tr}(\beta)=1$ (as a corollary, upon completion of the proof this shows that all bases equivalent to Type II Optimal normal bases are self-dual, see Section 2.6).

Let $\gamma$ be a root of the polynomial $x^{2}-\alpha x+1$ considered in $\mathbb{F}_{q^{2 n}}$ over $\mathbb{F}_{q^{n}}$ such that $\gamma+\gamma^{-1}=\alpha$. Let the order of $\gamma$ be $2 t+1$ for some $t$. For any integer $i$ let $\eta_{i}=\gamma^{i}+\gamma^{-i}$. Then $\eta_{i}=\eta_{j}$ if and only if $i \equiv \pm j(\bmod 2 t+1)$ and thus there are exactly $t$ non-zero distinct values of $\eta$. Any conjugate of $\alpha$ is of the form $\alpha^{2^{j}}=\gamma^{2^{j}}+\gamma^{-2^{j}}=\eta_{2 j}$ where $\eta_{2 j}$ is one of $\eta_{1}, \eta_{2}, \ldots, \eta_{t}$. Thus, the degree of $\alpha, n$, is at most $t$.

To show that $n=t$, observe that $\eta_{0}=0, \eta_{1}=\alpha$ and $\eta_{2}=\alpha^{2}$ so consider $i$ such that $3 \leq i \leq t$. We show by induction that $\eta_{i}$ is a conjugate of $\alpha$. Consider

$$
\alpha \eta_{i-2}=\left(\gamma+\gamma^{-1}\right)\left(\gamma^{i-2}+\gamma^{-(i-2)}\right)=\eta_{i-1}+\eta_{i-3}
$$

with $\eta_{i-1}$ conjugate to $\alpha$ and either $\eta_{i-3}$ conjugate to $\alpha$ (for $i \neq 3$ ) or $\eta_{i-3}=0$. The 
latter case proves the assertion, and the former case gives that $\eta_{i-1}$ occurs in the normal basis $N$ with a coefficient of 1 . Since $\beta=\alpha$ we have that the multiplication tables $T$ and $D$ are equal and symmetric. Therefore under a reordering of bases we have that $\alpha \eta_{i-1}$ is the sum of $\eta_{i-2}$ and another conjugate of $\alpha$. Since $\alpha \eta_{i-1}=\eta_{i-2}+\eta_{i}$, this gives that $\eta_{i}$ is a conjugate of $\alpha$ and thus every $\eta_{1}, \eta_{2}, \ldots, \eta_{t}$ are conjugates of $\alpha$.

For each integer $i$ not divisible by $2 n+1=2 t+1$ there is an integer $j$ such that $i \equiv \pm 2^{j}(\bmod 2 n+1)$. Since $2 n+1$ is odd, each $i$ is relatively prime to $2 n+1$ and so $2 n+1$ is prime, and $\mathbb{Z}_{2 n+1}$ is generated by 2 and -1 . This shows that the Optimal normal basis $N$ satisfies the second assertion in the statement; i.e: that $N$ is equivalent to a Type II Optimal normal basis.

\subsection{Distribution of Normal Bases}

The following theorem, due to Hensel [19], gives a criterion for determining if a field element is normal. This method is used extensively in Chapter 3. To prove this result we introduce a certain class of matrices whose structure is related to powering normal elements.

Definition 2.4.1. Let $\alpha_{1}, \alpha_{2}, \ldots, \alpha_{n} \in \mathbb{F}_{q^{n}}$, the circulant matrix on $\alpha_{1}, \alpha_{2}, \ldots, \alpha_{n}$ is 
given by

$$
\left(\begin{array}{cccc}
\alpha_{1} & \alpha_{2} & \cdots & \alpha_{n} \\
\alpha_{n} & \alpha_{1} & \cdots & \alpha_{n-1} \\
\vdots & \vdots & & \vdots \\
\alpha_{2} & \alpha_{3} & \cdots & \alpha_{1}
\end{array}\right)
$$

That is, each row in Equation (2.14) is a cyclic shift of the previous row.

Lemma 2.4.2. Let $A$ be the circulant matrix,

$$
A=\left(\begin{array}{cccc}
\alpha_{1} & \alpha_{2} & \cdots & \alpha_{n} \\
\alpha_{n} & \alpha_{1} & \cdots & \alpha_{n-1} \\
\vdots & \vdots & & \vdots \\
\alpha_{2} & \alpha_{3} & \cdots & \alpha_{1}
\end{array}\right)
$$

then $A$ is invertible if and only if $\sum_{i=1}^{n} \alpha_{i} x^{i}$ and $x^{n}-1$ are relatively prime.

Proof. Define the $n \times n$ matrix $P$ by

$$
P=\left(\begin{array}{ccccc}
0 & 1 & 0 & \cdots & 0 \\
0 & 0 & 1 & \cdots & 0 \\
\vdots & \vdots & \vdots & & \vdots \\
0 & 0 & 0 & \cdots & 1 \\
1 & 0 & 0 & \cdots & 0
\end{array}\right)
$$


and notice that

$$
A=\sum_{i=1}^{n} \alpha_{i} P^{i}
$$

Define $f(x)=\sum_{i=1}^{n} \alpha_{i} x^{i}$ then $A=f(P)$. By cofactor expansion we observe that the characteristic polynomial of $P$ is $x^{n}-1$. The matrix $P$ is a permutation matrix of order $n$ and the minimal polynomial of $P$ is $x^{n}-1$. Suppose $f$ is relatively prime to $x^{n}-1$. Then there are polynomials $a, b \in \mathbb{F}_{q}[x]$ such that

$$
f(x) a(x)+\left(x^{n}-1\right) b(x)=1 .
$$

Evaluating at $P$ and applying the Cayley-Hamilton theorem gives $f(P) a(P)=1$. That is, $A \cdot a(P)=1$ and $A$ is invertible.

Suppose to the contrary that $f(x)=\sum_{i=1}^{n} \alpha_{i} x^{i}$ and $x^{n}-1$ have a common divisor $d \in \mathbb{F}_{q}[x]$. Then $f(x)=g(x) d(x)$ and $x^{n}-1=h(x) d(x)$. Evaluating at $P$ we have $h(P) d(P)=0$, but since the degrees of $h, d$ are less than $n$, we have that $h(P), d(P) \neq 0$ and so $d(P)$ is not invertible. This implies that $A=g(P) d(P)$ is not invertible.

Theorem 2.4.3. For any $\alpha \in \mathbb{F}_{q^{n}}, \alpha$ is a normal element of $\mathbb{F}_{q^{n}}$ over $\mathbb{F}_{q}$ if and only if $\alpha^{q^{n-1}} x^{n-1}+\alpha^{q^{n-2}} x^{n-2}+\cdots+\alpha^{q} x+\alpha$ and $x^{n}-1$ are relatively prime.

Proof. By definition, $\alpha \in \mathbb{F}_{q^{n}}$ is a normal element if and only if $\alpha, \alpha^{q}, \alpha^{q^{2}}, \ldots, \alpha^{q^{n-1}}$ 
are linearly independent. By Corollary 2.1 .23 , this occurs if and only if the matrix

$$
\left(\begin{array}{cccc}
\alpha & \alpha^{q} & \cdots & \alpha^{q^{n-1}} \\
\alpha^{q} & \alpha^{q^{2}} & \cdots & \alpha \\
\vdots & \vdots & & \vdots \\
\alpha^{q^{n-1}} & \alpha^{q^{n-2}} & \cdots & \alpha
\end{array}\right)
$$

is non-singular.

It is clear that Equation (2.15) forms a circulant matrix. By Lemma 2.4.2, this is non-singular if and only if $\alpha^{q^{n-1}} x^{n-1}+\alpha^{q^{n-2}} x^{n-2}+\cdots+\alpha^{q} x+\alpha$ and $x^{n}-1$ are relatively prime.

We state without proof an important corollary we require to prove the structure of a dual element. The proof is quite short but requires some results about circulant matrices beyond the scope of this work; see $[10,22]$ for a rigorous discussion of these results.

Corollary 2.4.4. Let $\alpha \in \mathbb{F}_{q^{n}}, \alpha_{i}=\alpha^{q^{i}}$ and let $t_{i}=\operatorname{Tr}\left(\alpha_{0} \alpha_{i}\right)$. Then $\alpha$ generates $a$ normal basis of $\mathbb{F}_{q^{n}}$ over $\mathbb{F}_{q}$ if and only if $h(x)=t_{n-1} x^{n-1}+\cdots+t_{1} x+t_{0}$ and $x^{n}-1$ are relatively prime.

To establish the number of normal bases, we define an analog of Euler's $\phi$ function for polynomials.

Definition 2.4.5. [6] Let $f \in \mathbb{F}_{q}[x]$ be a polynomial of degree $m$. Then $\Phi(f)$ denotes the number of polynomials of degree less than $m$ that are relatively prime to $f$. 
From Theorem 2.4.3, it is clear that the number of normal elements in a finite field $\mathbb{F}_{q^{n}}$ over $\mathbb{F}_{q}$ is $\Phi\left(x^{n}-1\right) \in \mathbb{F}_{q}[x]$. Since each normal element and its conjugates form the same basis, the number of normal bases of $\mathbb{F}_{q^{n}}$ over $\mathbb{F}_{q}$ is $\frac{1}{n} \Phi\left(x^{n}-1\right)$. An explicit expression for the number of normal elements was established by Ore.

Theorem 2.4.6. [22] Let $q$ be a power of the prime $p$, let $n$ be a positive integer and write $n=p^{a} m$, where $p$ does not divide $m$. Then the number of normal bases of $\mathbb{F}_{q^{n}}$ over $\mathbb{F}_{q}$ equals

$$
\frac{1}{n} \Phi_{q}\left(x^{n}-1\right)=\frac{q^{n}}{n} \prod_{d \mid m}\left(1-q^{-o_{d}(q)}\right)^{\phi(d) / o_{d}(q)}
$$

where $\Phi_{q}(f)$ is the number of polynomials of degree smaller than the degree of $f$ which are relatively prime to $f, o_{n}(a)$ is the order of a modulo $n$, and $\phi(d)$ is the number of positive integers smaller than $d$ that are relatively prime to $d$.

Several authors have provided computationally feasible lower and upper bounds for the number of normal bases of $\mathbb{F}_{q^{n}}$ over $\mathbb{F}_{q}$. Here, we present Gao and Panario's bounds [11]:

$$
\frac{q^{n}}{e^{0.83} n\left(1+\log _{q} n\right)} \leq \frac{1}{n} \Phi_{q}\left(x^{n}-1\right) \leq \frac{q^{n}}{e^{\gamma-c_{q}} n \sqrt{1+\log _{q} n}}
$$

where $\gamma=0.577216 \ldots$ is Euler's constant and $c_{q}=q /(q-1)(\sqrt{q}-1)$; see also [9]. Von zur Gathen and Giesbrecht [16] showed that the probability that an arbitrary element in $\mathbb{F}_{q^{n}}$ forms a normal basis is larger than $1 /\left(16 \log _{q} n\right)$. 


\subsection{Constructions of Normal Bases}

Though normal bases are relatively dense in finite fields (see Chapter 3), it is nontrivial to find normal bases with prescribed properties, such as low-complexity. By constructing new normal bases from other known normal bases, we can inherit some of the desired properties from the known basis.

As the proof of Theorem 2.3.5 uses a projection from $\mathbb{F}_{2^{2 n}}$ to $\mathbb{F}_{2^{n}}$, the following theorem works in reverse and allows us to construct new normal bases with known complexity over particular extensions using previously known normal bases.

Theorem 2.5.1. $[10,35]$ Let $n=m k$, all positive integers with $m, k$ relatively prime. Let $\alpha \in \mathbb{F}_{q^{m}}$ and $\beta \in \mathbb{F}_{q^{k}}$ be normal elements over $\mathbb{F}_{q}$. Then $\gamma=\alpha \beta$ is a normal element of $\mathbb{F}_{q^{n}}$ over $\mathbb{F}_{q}$, with complexity equal to the product of the complexities of $\alpha$ and $\beta$.

Proof. Define $\alpha, \beta, \gamma$ as in the hypothesis. We must first show that the conjugates of $\gamma$ are linearly independent. We have $\gamma^{q^{i}}=\alpha^{q^{i}} \beta^{q^{i}}$. By the Chinese Remainder theorem, since $\operatorname{gcd}(m, k)=1$ there exist unique positive integers $\lambda, \mu$ such that $i \equiv \lambda$ $(\bmod m)$ and $i \equiv \mu(\bmod k)$, so $\gamma^{q^{i}}=\alpha^{q^{\lambda}} \beta^{q^{\mu}}$. Therefore, the conjugates of $\gamma$ are $\alpha^{q^{s}} \beta^{q^{t}}$ with $1 \leq s \leq m$, and $1 \leq t \leq k$. Suppose there are constants $a_{s t} \in \mathbb{F}_{q}$ such that

$$
\sum_{s, t} a_{s t} \alpha^{q^{s}} \beta^{q^{t}}=0
$$


and define $b_{t}=\sum_{s=0}^{m-1} a_{s t} \alpha^{q^{s}} \in \mathbb{F}_{q^{m}}$. Then

$$
\sum_{t=0}^{k-1} b_{t} \beta^{q^{t}}=0
$$

and we require the conjugates of $\beta$ to be linearly independent over $\mathbb{F}_{q^{m}}$.

For any integer $j$,

$$
\left(\sum_{t=0}^{k-1} b_{t} \beta_{t}\right)^{q^{j m}}=\sum_{t=0}^{k-1} b_{t}^{q^{j m}} \beta_{t}^{q^{j m}}=\sum_{t=0}^{k-1} b_{t} \beta_{t}^{q^{j m}}
$$

Since $m$ and $k$ are relatively prime, as $j$ runs from $0,1, \ldots, k-1$, then $j m$ runs from $0,1, \ldots, k-1(\bmod k)$. But this means that $\sum_{t=0}^{k-1} b_{t} \beta_{t}^{q^{j}}=0$ for $0 \leq j \leq k-1$. We construct the $k \times k$ matrix in expression Equation (2.15) using $\left(\beta_{0}, \beta_{1}, \ldots, \beta_{k-1}\right)$ as the first row. The matrix is invertible since $\beta_{0}, \beta_{1}, \ldots, \beta_{k-1}$ are linearly independent over $\mathbb{F}_{q}$. So $b_{0}=b_{1}=\cdots=b_{k-1}=0$ and $\beta_{0}, \beta_{1}, \ldots, \beta_{k-1}$ are linearly independent over $\mathbb{F}_{q^{m}}$.

Since $b_{t}=0$ for $0 \leq t \leq k-1$ we find

$$
\sum_{s=0}^{m-1} a_{s t} \alpha^{q^{s}}=0
$$

The conjugates of $\alpha$ are linearly independent over $\mathbb{F}_{q}$ and therefore $a_{s t}=0$ for all $0 \leq s \leq m-1,0 \leq t \leq k-1$, proving independence of the conjugates of $\gamma$.

Now, to show that the complexity of $\gamma$ is the product of the complexities of $\alpha$ and 
$\beta$, let $T_{\alpha}=\left(t_{i j}\right), T_{\beta}=\left(s_{u v}\right)$ be the multiplication table of $\alpha, \beta$ respectively. Then $\gamma \gamma^{q^{i}}=(\alpha \beta) \alpha^{q^{\lambda}} \beta^{q^{\mu}}$ by the Chinese Remainder theorem, as above. Using the common notation we find

$$
\begin{aligned}
\gamma \gamma_{i}=\alpha \alpha_{\lambda} \cdot \beta \beta_{\mu} & =\left(\sum_{j=0}^{m-1} t_{\lambda j} \alpha_{j}\right)\left(\sum_{v=0}^{k-1} s_{\mu v} \beta_{v}\right) \\
& =\sum_{j=0}^{m-1} \sum_{v=0}^{k-1} t_{\lambda j} s_{\mu v} \alpha_{j} \beta_{v} .
\end{aligned}
$$

Reapplying the Chinese Remainder theorem as above, there exists $\gamma_{l}=\alpha_{j} \beta_{v}$ for some $0 \leq l \leq n-1$ and for all $0 \leq j \leq m-1$ and $0 \leq v \leq k-1$ such that Equation (2.17) becomes

$$
\gamma \gamma_{i}=\sum_{j=0}^{m-1} \sum_{v=0}^{k-1} t_{\lambda j} s_{\mu v} \gamma_{l} .
$$

Consequently, the number of non-zero terms in the multiplication table of the basis generated by $\gamma$ is the product of the number of non-zero terms in $T_{\alpha}$ and $T_{\beta}$.

Corollary 2.5.2. Let $\mathbb{F}_{q^{n}}$ and $\mathbb{F}_{q^{m}}$ be finite extensions of $\mathbb{F}_{q}$ where $\operatorname{gcd}(m, n)=1$. Then

$$
\begin{aligned}
& \qquad C_{q}(m n) \leq C_{q}(m) C_{q}(n) . \\
& \text { If } \mathbb{F}_{q^{n}} \text { and } \mathbb{F}_{q^{m}} \text { contain optimal normal bases then } \\
& C_{q}(m n) \leq 4 m n-2(m+n)+1 .
\end{aligned}
$$

We present a more general theorem due to Ash, Blake and Vanstone for $q=2$, 
and independently proved by Beth, Geiselmann and Meyer for general $q$. The authors give a way of constructing a normal element with a low complexity, under certain conditions. We state the result for general $q$ and follow with specific results for $q=2$.

Theorem 2.5.3. $[1,29]$ Let $k$ and $n$ be positive integers. Assume $k n+1$ is a prime, and let $\gamma$ be a primitive $k$ th root of unity in $\mathbb{F}_{k n+1}$. If $q$ and $\gamma$ generate the multiplicative group $\mathbb{F}_{k n+1}^{*}$, and if $\xi$ is a primitive $(k n+1)$ th root of unity in $\mathbb{F}_{q^{k n}}$, then

$$
\alpha=\xi+\xi^{\gamma}+\cdots+\xi^{\gamma^{k-1}}
$$

generates a normal basis $N$ of $\mathbb{F}_{q^{n}} / \mathbb{F}_{2}$ with complexity $c_{N} \leq(k+1) n-k$. If $n$ is a multiple of $k$, this bound can be improved to $c_{N} \leq k n-1$.

Specific results can be obtained from Theorem 2.5.3 for the case $q=2$. We present some bounds, the lower bounds due to Ash, Blake and Vanstone and the upper bounds due to Beth, Geiselmann and Meyer.

Theorem 2.5.4. [22] Suppose $q=2$ in Theorem 2.5.3. Then

$$
\begin{aligned}
& (k+1) n-\left(k^{2}+k+1\right) \leq c_{N} \leq(k+1) n-2 k+1 \text {, if } k \text { is odd } \\
& k n-\left(k^{2}-3 k+3\right) \quad \leq c_{N} \leq k n-k+1, \quad \text { if } k \text { is even. }
\end{aligned}
$$

Ash, Blake and Vanstone also showed that the lower bounds in the above theorem are quite often sharp. More precisely, it holds for all sufficiently large $n$ as long as $k$ is a power of 2 , an odd prime, twice an odd prime, or four times an odd prime. 
Meyer showed that for $k \leq 7$ the lower bounds hold with just four exceptions.

For $q=2$ and specific $n, k$ the bases constructed using this theorem have prescribed complexity. Some results are presented below.

Theorem 2.5.5. [22] Suppose $q=2$ in Theorem 2.5.3. Then for specific values of $n, k$ we find

$$
c_{N}= \begin{cases}4 n-7, & \text { for } k=3 \text { and } k=4 \\ 6 n-21, & \text { for } k=5 \text { and } n>2, \text { and for } k=6 \text { and } n>6 \\ 8 n-43, & \text { for } k=7 \text { and } n>4 .\end{cases}
$$

We present direct constructions of low complexity normal bases of $\mathbb{F}_{q^{n}}$ over $\mathbb{F}_{q}$ by Blake, Gao and Mullin using the theory of linear fractional forms. These results are mainly interesting for large $q$.

Theorem 2.5.6. [3] For every $a, \beta \in \mathbb{F}_{q}^{*}$ with $\operatorname{Tr}_{\mathbb{F}_{q} / \mathbb{F}_{p}}(\beta)=1$,

$$
x^{p}-\frac{1}{\beta} a x^{p-1}-\frac{1}{\beta} a^{p}
$$

is irreducible over $\mathbb{F}_{q}$ and its roots form a normal basis of $\mathbb{F}_{q^{p}}$ over $\mathbb{F}_{q}$ with complexity at most $3 p-2$.

Theorem 2.5.7. [3] Let $n$ be any factor of $q-1$. Let $\beta \in \mathbb{F}_{q}$ with multiplicative order 
$t$ such that $n$ is relatively prime to $\frac{q-1}{t}$ and let $a=\beta^{(q-1) / n}$. Then

$$
x^{n}-\beta(x-a+1)^{n}
$$

is irreducible over $\mathbb{F}_{q}$ and its roots form a normal basis of $\mathbb{F}_{q^{n}}$ over $\mathbb{F}_{q}$ with complexity at most $3 n-2$.

We present some new constructions of low complexity normal bases using projections from optimal extension fields in Chapter 4.

\subsection{The Dual of a Normal Basis}

We begin by giving the classical definition of a dual vector space.

Definition 2.6.1. Let $V$ be a vector space over a field $F$. The dual space of $V$ is the space of all linear transformations from $V$ into $F$, denoted $\operatorname{Hom}_{F}(V, F)$

We present the dual basis for the case of finite fields. Many of the results hold more generally over any finite dimensional vector space; for a discussion of these results, see [20].

Definition 2.6.2. Let $N=\left\{\alpha_{0}, \alpha_{1}, \ldots, \alpha_{n-1}\right\}$ be a basis of $\mathbb{F}_{q^{n}}$ over $\mathbb{F}_{q}$ and let $M=$ $\left\{\beta_{0}, \beta_{1}, \ldots, \beta_{n-1}\right\}$ be another basis. Then $M$ is the dual basis of $N$ if $\operatorname{Tr}\left(\alpha_{i} \beta_{j}\right)=\delta_{i j}$ for $0 \leq i, j \leq n-1$, where $\delta$ is the Kronecker delta function. A basis as called self-dual if it is a dual of itself, i.e. $N$ is self-dual if $\operatorname{Tr}\left(\alpha_{i} \alpha_{j}\right)=\delta_{i j}$ for all $i, j$. 
Theorem 2.6.3. Let $N=\left\{\alpha_{0}, \alpha_{1}, \ldots, \alpha_{n-1}\right\}$ be a basis of $\mathbb{F}_{q^{n}}$ over $\mathbb{F}_{q}$ and let $M=$ $\left\{\beta_{0}, \beta_{1}, \ldots, \beta_{n-1}\right\}$ be a dual basis of $N$. Then $M$ is the unique dual basis of $N$.

Proof. Let $N=\left\{\alpha_{0}, \alpha_{1}, \ldots, \alpha_{n-1}\right\}$ be a basis of $\mathbb{F}_{q^{n}}$ over $\mathbb{F}_{q}$ and $M=\left\{\beta_{0}, \beta_{1}, \ldots\right.$, $\left.\beta_{n-1}\right\}$ be a dual basis of $N$ for the dual vector space $\operatorname{Hom}_{\mathbb{F}_{q}}\left(\mathbb{F}_{q^{n}}\right)$. Then $M$ consists of the $n$ linear mappings from $\mathbb{F}_{q^{n}}$ to $\mathbb{F}_{q}$ defined by $\beta_{i}\left(\alpha_{j}\right)=\delta_{i j}$ where $\delta_{i j}$ is the Kronecker delta function. By Theorem 2.1.19 there is an isomorphism from $\mathbb{F}_{q^{n}}$ to the homomorphisms of $\mathbb{F}_{q^{n}}$ into $\mathbb{F}_{q}$. Define $\gamma_{i}$ to be the pre-image of $\beta_{i}$ under this isomorphism, $0 \leq i \leq n-1$, and let $C=\left\{\gamma_{0}, \gamma_{1}, \ldots, \gamma_{n-1}\right\}$. Since isomorphisms preserve linear independence, $C$ is a basis of $\mathbb{F}_{q^{n}}$ over $\mathbb{F}_{q}$, and the construction of these isomorphisms guarantees $\operatorname{Tr}\left(\beta_{i} \gamma_{j}\right)=\delta_{i j}$ for $0 \leq i, j \leq n-1$. This uniquely determines $C$, and therefore uniquely determines $M$.

Theorem 2.6.4. Let $N=\left\{\alpha_{0}, \alpha_{1}, \ldots, \alpha_{n-1}\right\}$ be a normal basis of $\mathbb{F}_{q^{n}}$ over $\mathbb{F}_{q}$, and $M=\left\{\beta_{0}, \beta_{1}, \ldots, \beta_{n-1}\right\}$ be the dual basis of $N$. Then $M$ is a normal basis of $\mathbb{F}_{q^{n}}$ over $\mathbb{F}_{q}$.

Proof. We have that $M$ is a basis, so it remains to show that $M$ is generated by the conjugates of $\beta_{0}$. We show, by a similar argument to the above, that the $i$ th conjugate of $\beta_{0}$ induce the same linear mapping from $\mathbb{F}_{q^{n}}$ onto $\mathbb{F}_{q}$ as $\beta_{i}$. By Theorem 2.1.19 this guarantees that they are the same element.

Since $M$ is a dual basis we have $\operatorname{Tr}\left(\alpha_{i} \beta_{j}\right)=\delta_{i j}$ for $0 \leq i, j \leq n-1$. Consider the 
special case $j=0$, and apply the Frobenius automorphism $\phi_{q}$ to obtain

$$
\operatorname{Tr}\left(\beta_{i+1} \gamma_{0}^{q}\right)=\operatorname{Tr}\left(\left(\beta_{i} \gamma_{0}\right)^{q}\right)=\operatorname{Tr}\left(\beta_{i} \gamma_{0}\right)=\delta_{i 0}
$$

for $0 \leq i \leq n-1$. This shows that $\gamma_{1}$ and $\gamma_{0}^{q}$ induce the same mapping. By reapplying this argument for the first $n-1$ powers of the Frobenius automorphism this proves $\gamma_{j}=\gamma_{0}^{q^{j}}$ for $0 \leq j \leq n-1$.

Lemma 2.6.5. Let $N=\left\{\alpha_{0}, \alpha_{1}, \ldots, \alpha_{n-1}\right\}$ be a normal basis of $\mathbb{F}_{q^{n}}$ over $\mathbb{F}_{q}$ with multiplication table $T=\left(t_{i j}\right)$ and let $M=\left\{\beta_{0}, \beta_{1}, \ldots, \beta_{n-1}\right\}$ be the dual basis of $N$ with multiplication table $D=\left(d_{i j}\right)$. Then $\left(d_{i j}\right)=\left(t_{j i}\right)$.

Proof. By definition of the dual basis, we have

$$
\operatorname{Tr}\left(\alpha_{i} \beta_{j}\right)= \begin{cases}1 & \text { if } i=j \\ 0 & \text { if } i \neq j\end{cases}
$$

. We consider the quantity

$$
\operatorname{Tr}\left(\alpha \beta_{i} \alpha_{k}\right)=\operatorname{Tr}\left(\sum_{j=0}^{n-1} d_{i j} \beta_{j} \alpha_{k}\right)=\sum_{j=0}^{n-1} d_{i j} \operatorname{Tr}\left(\beta_{j} \alpha_{k}\right)=d_{i k}
$$

but we also find

$$
\operatorname{Tr}\left(\alpha \beta_{i} \alpha_{k}\right)=\operatorname{Tr}\left(\sum_{j=0}^{n-1} t_{k j} \alpha_{j} \beta_{i}\right)=\sum_{j=0}^{n-1} t_{k j} \operatorname{Tr}\left(\alpha_{j} \beta_{i}\right)=t_{k i}
$$


This shows that the multiplication table for the dual basis is the transpose of the multiplication table of the normal basis.

Gao showed that the dual basis of a normal basis can be computed explicitly. This result is used in Chapter 4 to determine the complexity of a given basis.

Theorem 2.6.6. [10] Let $N=\left\{\alpha_{0}, \alpha_{1}, \ldots, \alpha_{n-1}\right\}$ be a normal basis of $\mathbb{F}_{q^{n}}$ over $\mathbb{F}_{q}$ and let $t_{i}=\operatorname{Tr}\left(\alpha_{0} \alpha_{i}\right)$. Let $h(x)=t_{n-1} x^{n-1}+\cdots+t_{1} x+t_{0}$ and let $g(x)=$ $d_{0}+d_{1} x+\cdots+d_{n-1} x^{n-1}$ be the unique monic polynomial of degree less than $n$ such that

$$
g(x) h(x) \equiv 1 \quad\left(\bmod x^{n}-1\right)
$$

The dual basis of $N$ is generated by the element

$$
\gamma=d_{0} \alpha_{0}+d_{1} \alpha_{1} \cdots+d_{n-1} \alpha_{n-1}
$$

Proof. Define $N, h(x), g(x), \gamma$ as in the hypothesis. Corollary 2.4 .4 gives that $h$ is invertible $\left(\bmod x^{n}-1\right)$, so $g$ is well-defined. The condition that $g(x) h(x) \equiv 1$ $\left(\bmod x^{n}-1\right)$ gives

$$
\sum_{k=0}^{n-1} d_{k} t_{i-k}= \begin{cases}1 & \text { if } i=0 \\ 0 & \text { if } i \neq 0\end{cases}
$$


and we find for any $0 \leq j \leq n-1$ with $\gamma_{j}=\gamma^{q^{j}}$

$$
\begin{aligned}
\operatorname{Tr}\left(\alpha_{i} \gamma_{j}\right) & =\operatorname{Tr}\left(\alpha_{i} \sum_{k=0}^{n-1} d_{k} \alpha_{k+j}\right)=\sum_{k=0}^{n-1} d_{k} \operatorname{Tr}\left(\alpha_{i} \alpha_{k+j}\right) \\
& =\sum_{k=0}^{n-1} d_{k} \operatorname{Tr}\left(\alpha_{i-j-k} \alpha_{0}\right)=\sum_{k=0}^{n-1} d_{k} t_{i-j-k}=\delta_{i j} .
\end{aligned}
$$

Thus, the conjugates of $\gamma$ form the dual basis of $N$.

We end this section by presenting some results about self-dual normal bases which are useful when considering the results in Chapter 3.

Proposition 2.6.7. The multiplication table of a self-dual normal basis is symmetric. Furthermore, any Type II Optimal normal basis of $F_{2^{n}}$ over $\mathbb{F}_{2}$ is self-dual.

This is a consequence of the proof of Theorem 2.3.10.

Theorem 2.6.8. [22] A self-dual normal basis of $\mathbb{F}_{q^{n}}$ over $\mathbb{F}_{q}$ exists if and only if either $q$ is even and $n$ is not a multiple of 4 or both $q$ and $n$ are odd.

As a consequence, there is no self dual normal basis over $\mathbb{F}_{2^{n}}$ if 4 divides $n$. If $n \equiv 2(\bmod 4)$, the next two results show how to obtain a self-dual normal basis over $\mathbb{F}_{2^{n}}$, provided that a basis of the same type is given, and how their complexities are related.

Proposition 2.6.9. [22] Let $\alpha \in \mathbb{F}_{2^{n}}$, where $n$ is even, and put $\gamma:=1+\alpha$. Then $\alpha$ generates a self-dual normal basis if and only if $\gamma$ does. 
Proposition 2.6.10. [22] Let $\alpha$ generate a self-dual normal basis $B$ for $\mathbb{F}_{2^{n}}$ over $\mathbb{F}_{2}$, and assume that $n$ is even. Put $\gamma:=1+\alpha$, and let $B^{C}$ be the self-dual normal basis generated by $\gamma$. Then the complexities of $B$ and $B^{C}$ are related as follows

$$
C_{B^{C}}=n^{2}-3 n+8-C_{B}
$$

Corollary 2.6.11. [22] For $n \equiv 2(\bmod 4)$, the average complexity of a self-dual normal basis for $\mathbb{F}_{2^{n}}$ over $\mathbb{F}_{2}$ is $\frac{1}{2}\left(n^{2}-3 n+8\right)$. Also, if $c_{B}$ is the complexity of a self-dual normal basis $B$ then

$$
2 n-1 \leq c_{B} \leq n^{2}-5 n+9
$$

Equality holds in one of these bounds if and only if either $B$ or $B^{C}$ is optimal. 


\section{Chapter 3}

\section{Distribution of Complexities}

We know that optimal normal bases do not exist in every finite field $\mathbb{F}_{q^{n}}$ over $\mathbb{F}_{q}$, see Section 2.3.2. In these fields, the problem of finding the lowest complexity normal bases still remains. Ideally, we would like to find an upper-bound with the degree $n$ of the extension on the minimum complexity of an element in a finite field.

For a better understanding of the behavior of the complexities of normal elements in $\mathbb{F}_{2^{n}}$, tables summarizing the complexity distribution are important tools. In [22], Jungnickel provides a table with minimum and maximum complexities of normal elements in $\mathbb{F}_{2^{n}}$, for each $n$ smaller than or equal to 30 . He also provides a table due to Geiselmann [17] with the lowest complexities found via free polynomials.

In this chapter, we present the distribution of the complexity of normal elements for $n \leq 39$. We gather, for each $n$ in that range, the frequency of each possible complexity value. Summary tables with minimum, maximum, average and variance values for the complexities of normal elements and self-dual normal elements in $\mathbb{F}_{2^{n}}$ 
are found in Appendix B. Our data is obtained via an exhaustive search in $\mathbb{F}_{2^{n}}$ which is substantially sped up by visiting the finite field elements in a Gray code order. An extension of the table of the smallest known complexities is given in Appendix C for other practical values of interest $(40 \leq n \leq 607)$, using a combination of known results and a random search.

\subsection{Exhaustive Search of Normal Bases}

In this section, we describe and analyze two variants of an exhaustive algorithm for computing the complexity of each normal element in $\mathbb{F}_{q^{n}}$. Algorithm StandardNCD (for "Standard Normal Complexity Distribution") is the simplest variant. Algorithm GrayCodeNCD (for "Gray Code Normal Complexity Distribution"), the second variant, uses Gray codes in order to efficiently update the current finite field element and its conjugates. We use basic operations in $\mathbb{F}_{q}$ as our time complexity measure. We show that asymptotically the Gray code variant reduces the time complexity of the standard algorithm by $28.6 \%$. We present the algorithms and their analyses for $q=2$, but their extensions to general $q$ are straightforward.

\subsubsection{Description of the Algorithms}

We now describe Algorithm StandardNCD; its pseudocode appears below. At each step, we compute $\alpha \in \mathbb{F}_{2^{n}}$, represented as a polynomial over $\mathbb{F}_{2}$ with coefficients stored in the tuple $\alpha_{\text {coeff }}=\left[a_{n-1}, \ldots, a_{0}\right]$. Variables $\alpha_{j}=\alpha^{2^{j}}, j=0,1, \ldots, n-1$, 
store $\alpha$ and its conjugates. The main loop runs through $\alpha \in \mathbb{F}_{2^{n}}^{*}$ by updating the tuple $\alpha_{\text {coeff }}$ with the next binary tuple using an arbitrary order computed via function NextTuple() in line 12.

Each field element $\alpha$ is processed in lines 6-11. Since conjugates form an equivalence class for the computation of the complexity of normal elements, we only compute the complexity for one canonical representative of the normal basis. We stipulate that $\alpha$ is canonical if and only if $\alpha$ is the unique minimum element among $\left\{\alpha^{2^{j}}\right\}_{j=0}^{n-1}$, when using the lexicographical ordering of the coefficients of their polynomial representations. For a canonical element $\alpha$, we check if it is normal using procedure IsNormal which employs a gcd test based on Theorem 2.4.3. If $\alpha$ is normal, then we calculate its complexity via procedure CalculateComplexity, which is an implementation of the complexity definition. This amounts to solving an $n \times n$ system of equations with coefficients in $\mathbb{F}_{2}$. Since the only difference between these systems of equations are their right-hand sides, we calculate the inverse of the system's matrix (matrix $P$ ) and then apply $n$ multiplications of $P$ by vectors in $\mathbb{F}_{2^{n}}$.

The values of $\alpha$ and its conjugates are updated in lines 12-15. Algorithm Standard NCD does a straight-forward update, while Algorithm GrayCodeNCD employs a more efficient update that uses a Gray code. 


\subsubsection{Gray Codes}

A Gray code is a sequential ordering of the $2^{n}$ binary vectors of length $n$ such that any two consecutive vectors have Hamming distance equal to one, i.e. any two consecutive vectors differ in a unique position $k$. Gray codes are well studied (see [34]) and they exist for every $n$. In particular, we can build a Gray code in which the first vector is the zero vector, and each subsequent vector can be computed in time linear with $n$. In GrayCodeNCD algorithm, this computation is done by calling function NextGrayTuple() in line 12. There are multiple possible Gray Codes for a given $n$, one possible algorithm is given below.

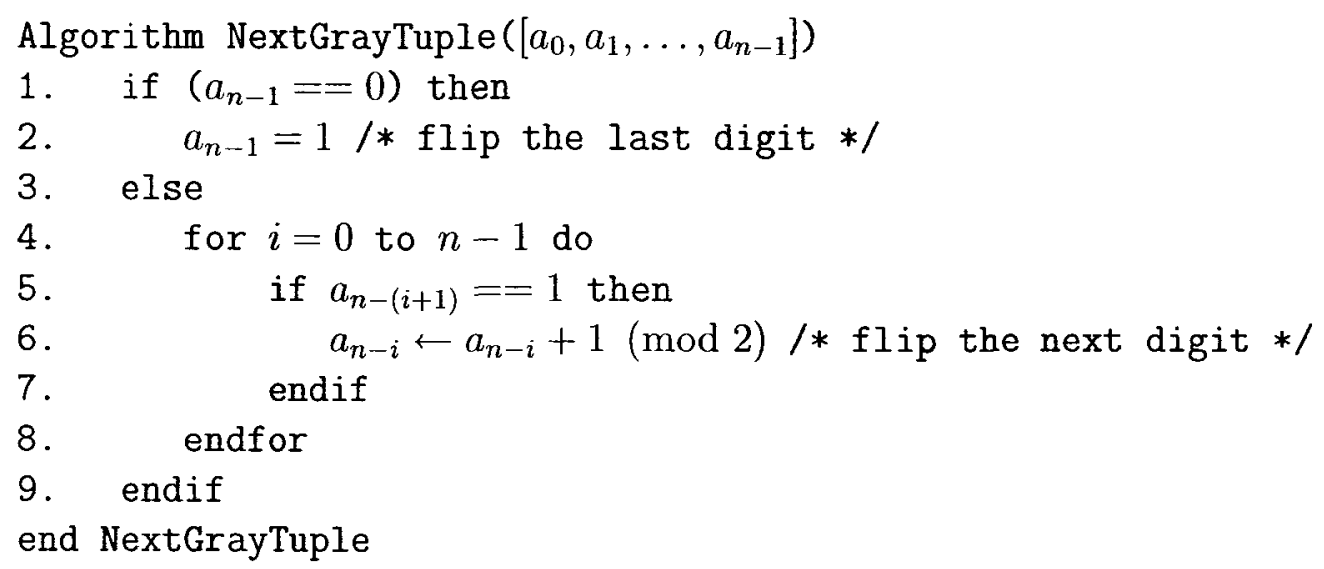

Now we describe how Gray codes are used to update the field element $\alpha \in \mathbb{F}_{2^{n}}$ in the GrayCodeNCD algorithm. Let $\alpha^{\prime}$ be the field element in the previous iteration of the Gray code and $\alpha$ be the field element in the current iteration, represented as polynomials in $\mathbb{F}_{2}[x]$. We represent the polynomial coefficients as the successive tuples in a Gray code, so we have $\alpha=\alpha^{\prime}+x^{k}$, for some $k \in\{0,1, \ldots, n-1\}$. Therefore, $\alpha^{2^{j}}=\left(\alpha^{\prime}+x^{k}\right)^{2^{j}}=\left(\alpha^{\prime}\right)^{2^{j}}+\left(x^{k}\right)^{2^{j}}$. In lines 1-2 of algorithm GrayCodeNCD, the values 
$e_{i}^{j}=\left(x^{i}\right)^{2^{j}}$ are precomputed, and used in line 15 for updating $\alpha$. This reduces the computation in lines 14-15 from $n-1$ squarings, as in the standard algorithm, to $n$ additions in the Gray code variant.

\subsubsection{StandardNCD and GrayCodeNCD Algorithms}

Now we present the StandardNCD and GrayCodeNCD algorithms.

Algorithm StandardNCD $(n)$

1. $\alpha_{\text {coeff }}=\operatorname{NextTuple}([0,0, \ldots, 0])$;

2. $\alpha_{0}=$ polynomial $\left(\alpha_{\text {coeff }}\right) ; / *$ initialize $\alpha * /$

3. for $j=1$ to $n-1$ do

4. $\quad \alpha_{j}=\left(\alpha_{j-1}\right)^{2} ; / *$ calculate conjugates $\alpha_{j}=(\alpha)^{2^{j}} * /$

5. for $i=1$ to $2^{n}-1$ do $/ *$ run through $\mathbb{F}_{2^{n}}^{*} * /$

6. if $\left(\left(\alpha_{0}, \alpha_{1}, \ldots, \alpha_{n-1}\right)\right.$ is canonical) then

7. if (IsNormal $\left(\alpha_{0}, \alpha_{1}, \ldots, \alpha_{n-1}\right)$ ) then

8. $\quad$ Compl=CalculateComplexity $\left(\alpha_{0}, \alpha_{1}, \ldots, \alpha_{n-1}\right)$;

9. UpdateStats $\left(\alpha_{0}\right.$, Compl)

10. endif

11. endif

12. $\alpha_{\text {coeff }}=\operatorname{NextTuple}\left(\alpha_{\text {coeff }}\right) ; / *$ go to next $* /$

13. $\alpha_{0}=$ polynomial $\left(\alpha_{\text {coeff }}\right)$;

14. for $j=1$ to $n-1$ do

15. $\alpha_{j}=\left(\alpha_{j-1}\right)^{2} ; / *$ calculate $\alpha_{j}=\alpha^{2^{j}} * /$

16. endfor

17. PrintFinalstats();

end StandardNCD

Algorithm GrayCodeNCD $(n)$

1. for $i=0$ to $n-1$ do $e_{i}^{0}=x^{i} ; / *$ precomputation $* /$

2. for $j=1$ to $n-1$ do $e_{i}^{j}=\left(e_{i}^{j-1}\right)^{2} ; / *$ precomputation */

3. $\alpha_{\text {coeff }}=$ NextGrayTuple $([0,0, \ldots, 0])=[0, \ldots, 0,1]$;

4. for $j=0$ to $n-1$ do $\alpha_{j}=e_{0}^{j}$;/* initial. $\alpha$ \& conjug.*/

5. for $i=1$ to $2^{n}-1$ do $/ *$ run through $\mathbb{F}_{2^{n}}^{*} * /$

6. if $\left(\left(\alpha_{0}, \alpha_{1}, \ldots, \alpha_{n-1}\right)\right.$ is canonical) then

7. if IsNormal $\left(\alpha_{0}, \alpha_{1}, \ldots, \alpha_{n-1}\right)$ then

8. Compl=CalculateComplexity $\left(\alpha_{0}, \alpha_{1}, \ldots, \alpha_{n-1}\right)$;

9. UpdateStats $\left(\alpha_{0}\right.$, Compl)

10. endif 
11. endif

12. $\alpha_{\text {coeff }}^{\prime}=\alpha_{\text {coeff }} ; \alpha_{\text {coeff }}=\operatorname{NextGrayTuple}\left(\alpha_{\text {coeff }}^{\prime}\right) ; / *$ next $* /$

13. Let $k$ be the index $\ell$ such that $\alpha_{\text {coeff }}[\ell] \neq \alpha_{\text {coeff }}^{\prime}[\ell]$;

14. for $j=0$ to $n-1$ do

15. $\alpha_{j}=\alpha_{j}+e_{k}^{j} ; / *$ efficient $\alpha_{j}$ computation $* /$

16. endfor

17. PrintFinalstats();

end GrayCodeNCD

Procedure CalculateComplexity $\left(\alpha^{0}, \alpha^{1}, \ldots, \alpha^{n-1}\right)$

1. for $i=0$ to $n-1$ do

2. $\beta^{i}=\alpha^{0} * \alpha^{i}$;

3. for $i=0$ to $n-1$ do

4. for $j=0$ to $n-1$ do

5. $\quad P_{i j}=j$-th coefficient of $\alpha^{i}$;

6. $A_{i j}=j$-th coefficient of $\beta^{i}$;

7. endfor

8. Calculate $P_{i n v}=P^{-1}$;

9. Calculate $T=A \times P_{\text {inv }} ; / * T=\left(t_{i j}\right) * /$

10. Compl $=\sum_{i=0}^{n-1} \sum_{j=0}^{n-1} t_{i j}$;

11. return Compl;

end CalculateComplexity

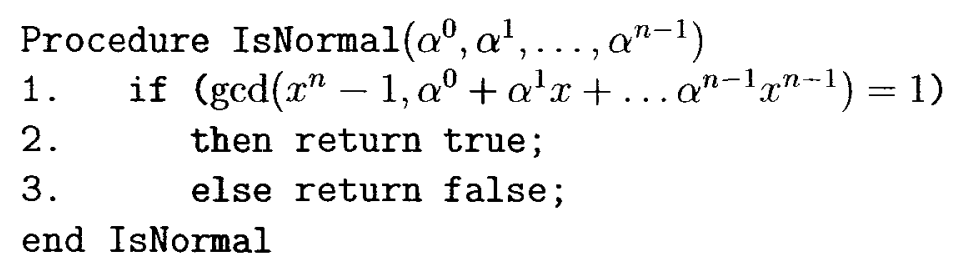

We recall Proposition 2.6.7: that the multiplication table of a self-dual normal bases is symmetric. Therefore, in order to adapt the given algorithms to search exclusively for self-dual normal elements, it is enough to alter the CalculateComplexity procedure to solve for $t_{i j}$ row-by-row (i.e. solving the system up to $n$ times), and check the non-diagonal elements (up to the index of the calculated row) for symmetry. If this follows to completion, the normal element is self-dual and the complexity 
is stored. We call this algorithm SelfDualNCD. A further refinement of this algorithm, considered after implementation, is due to Proposition 2.6.9: when $n$ is even, $\alpha$ generates a self-dual normal basis if and only if $\alpha+1$ generates a self-dual normal basis. If implemented, for $n \equiv 2(\bmod 4)$, the running time should be reduced approximately by half.

\subsubsection{Karatsuba Multiplication Scheme}

Elements in a finite field are classically represented as polynomials. Let $A, B \in$ $\mathbb{F}_{q^{n}}$ be represented as $a, b \in \mathbb{F}_{q}[x]$, where $a(x)=a_{n-1} x^{n-1}+\cdots+a_{1} x+a_{0}$ and $b(x)=b_{n-1} x^{n-1}+\cdots+b_{1} x+b_{0}$ where $a_{0}, b_{0}, a_{1}, b_{1}, \ldots, a_{n-1}, b_{n-1} \in \mathbb{F}_{q}$. Consider multiplication $A \cdot B=a(x) \cdot b(x)=c(x)=c_{n-1} x^{n-1}+\cdots+c_{1} x+c_{0}$ for some $c_{n-1}, \ldots, c_{1}, c_{0} \in \mathbb{F}_{q}$

The resultant $c$ is given as the residue modulo the reduction polynomial $f \in \mathbb{F}_{q}[x]$ where $\mathbb{F}_{q^{n}}=\mathbb{F}_{q} /(f)$. Classically, this is computed as term-by-term multiplication of $a$ and $b$ followed by the reduction step. This is classically performed in time $O\left(n^{2}\right)$.

Karatsuba and Ofman (1963) presented the first multiplication scheme for integers which runs in time less than $O\left(n^{2}\right)$ [23]. More precisely, Karatsuba presented a fast squaring scheme and noted the identity

$$
a b=1 / 4\left((a+b)^{2}+(a-b)^{2}\right)
$$

We present Karatsuba's multiplication scheme for finite fields of characteristic two [8]. 
We express the polynomial $A \in \mathbb{F}_{2^{n}}$ in two parts $A=A_{1} x^{n / 2}+A_{0}$. Consider

$$
\begin{aligned}
C & =A \cdot B=\left(A_{1} x^{n / 2}+A_{0}\right)\left(B_{1} x^{n / 2}+B_{0}\right) \\
& =A_{1} B_{1} x^{n}+\left(A_{1} B_{0}+A_{0} B_{1}\right) x^{n / 2}+A_{0} B_{0} .
\end{aligned}
$$

To simplify this expression we define

$$
\begin{aligned}
T_{1} & =A_{1} B_{1} \\
T_{2} & =\left(A_{1}+A_{0}\right)\left(B_{1}+B_{0}\right) \\
& =A_{1} B_{0}+A_{0} B_{1}+A_{1} B_{1}+A_{0} B_{0} \\
T_{3} & =A_{0} B_{0} .
\end{aligned}
$$

which reduces the expression to

$$
C=A B=T_{1} x^{n}+\left(T_{2}-T_{1}-T_{3}\right) x^{n / 2}+T_{3}
$$

This gives that an $n$ bit multiplication can be expressed by $3 n / 2$ bit multiplications and some additional additions.

We conclude this discussion by giving the time complexity of the Karatsuba multiplication scheme.

Theorem 3.1.1. [14] Karatatsuba's algorithm for multiplying polynomials of degree less than a power $n$ of 2 over a ring can be done with at most $9 n^{\log _{2} 3}$ or $O\left(n^{1.59}\right)$ ring 
operations.

\subsubsection{Analyses of the Algorithms}

In this section, we analyze the amortized worst-case running time per finite field element, which we call $T S(n)$ and $T G(n)$, for standard and Gray code variants, respectively. Therefore, the total running time of each algorithm will be at most $2^{n r} T S(n)$ and $2^{n} T G(n)$, respectively. We do our analysis in terms of $M(n)$, the number of basic steps in $\mathbb{F}_{2}$ used in a multiplication of polynomials of degree smaller than $n$ over $\mathbb{F}_{2}$. We then compute the savings in running time given by the Gray code variant with respect to the standard one.

We analyze the steps that are common to both algorithms. The canonicity test in line 6 of both algorithms can be done in $n^{2}$ comparisons in $\mathbb{F}_{2}$. Procedure IsNormal called in line 7 is only run for $1 / n$ of the field elements. Each time it is run, it involves the computation of a gcd between polynomials of degrees $n-1$ and $n$ over $\mathbb{F}_{2^{n}}$, see Theorem 2.4.3. This gcd can be computed via the classical method in time $(5 / 2) n^{2} M(n)+O(n)$ operations in $\mathbb{F}_{2}$, see [14]. Therefore the amortized time for line 7 is $(5 / 2) n M(n)+O(1)$.

The number of normal elements is $\Phi_{2}\left(x^{n}-1\right)$ by Theorem 2.4.6 therefore lines 8-9 are only executed $\Phi_{2}\left(x^{n}-1\right) / n$ times in total. Each execution of these lines runs the CalculateComplexity algorithm. For this algorithm, steps 1-2 take $O(n M(n))$, steps 3-7 and 9-10 take $O\left(n^{2}\right)$ and step 8 takes $O\left(n^{3}\right)$. In the classical multiplication scheme 
$M(n)=n^{2}$, and for Karatsuba's multiplication scheme $M(n)=n^{1+\log _{2} 3} \leq n^{1.59}$. There are other asymptotically faster multiplication schemes but for the sizes of $n$ we are considering it suffices to consider these two schemes, see [15]. So, the running time of CalculateComplexity is $O\left(n^{3}\right)$. Thus, the amortized cost for steps 8-9 is in $O\left(n^{3} \Phi_{2}\left(x^{n}-1\right) /\left(n 2^{n}\right)\right)$, which, by the upper bound in Equation (2.16), is contained in $O\left(n^{2} / \sqrt{\log n}\right)$.

The iterations of the StandardNCD and GrayCodeNCD algorithms differ in the updates in lines 12-16. For the standard algorithm, the cost of these lines is dominated by $n-1$ squarings of polynomials of degree smaller than $n$ over $\mathbb{F}_{2}$, which are done in $(n-1) M(n)$ operations in $\mathbb{F}_{2}$. For the Gray code algorithm, the cost of lines 12-16 is dominated by $n$ additions of polynomials of degree smaller than $n$ over $\mathbb{F}_{2}$, which is done in time $O\left(n^{2}\right)$, see [14].

Combining the above analyses for the steps of each algorithm, we get

$$
\begin{aligned}
T S(n) & =\frac{5}{2} n M(n)+n M(n)+O\left(n^{2}\right)=\frac{7}{2} n M(n)+O\left(n^{2}\right) \\
T G(n) & =\frac{5}{2} n M(n)+O\left(n^{2}\right) .
\end{aligned}
$$

Using the above equations, we conclude that the Gray code variant gives (asymptotically) savings of $\left(\frac{7}{2}-\frac{5}{2}\right) n M(n) /\left(\frac{7}{2} n M(n)\right) \approx 28.6 \%$.

We conclude with Table 3.1 where we give asymptotic estimates of $T S(n)$ and $T G(n)$ using classical and Karatsuba's multiplication methods. 


\begin{tabular}{|l|l|l|}
\hline Method & Karatsuba's & Classical \\
\hline$T S(n)$ & $31.5 n^{1+\log _{2} 3}+O\left(n^{2}\right)$ & $3.5 n^{3}+O\left(n^{2}\right)$ \\
$T G(n)$ & $22.5 n^{1+\log _{2} 3}+O\left(n^{2}\right)$ & $2.5 n^{3}+O\left(n^{2}\right)$ \\
\hline
\end{tabular}

Table 3.1: Running time of StandardNCD and GrayCodeNCD using Karatsuba and classical multiplication methods.

\subsection{Results}

The experiments were performed on individual Pentium IV $3.0 \mathrm{GHz}$ systems with 1.5 GB of DDR RAM. The operating system was Red Hat Linux Enterprise Edition kernel 2.6.9-34.EL. $\mathrm{C}++$, compiled using $\mathrm{g}++3.4 .3$, was used for all programming. For arithmetic performed over $\mathbb{F}_{2}$, Shoup's NTL package version 5.3.2 was used [36], specifically taking advantage of the optimized binary arithmetic "GF2" libraries. For $n=37,38,39$ the Gray code was split into multiple sections and run simultaneously across many machines which reduces the run-time linearly with the number of machines used.

\subsubsection{Running Time Comparison}

Table 3.2 gives the CPU User-time for the StandardNCD, GrayCodeNCD and the SelfDual-

NCD respectively, measured in seconds, followed by a figure for graphical comparison.

We observe from Table 3.2 that the running time of the SelfDualNCD algorithm is approximately ten percent faster than the GrayCodeNCD algorithm running time, and so it was not included in Figure 3.1. Moreover, a comparison of the running time per 


\begin{tabular}{|r|r|r|r|r|r|r|r|}
\hline $\mathrm{n}$ & Standard & GrayCode & SelfDual \\
\hline 2 & 0.00 & 0.00 & $\mathbf{0 . 0 0}$ \\
3 & 0.00 & 0.00 & 0.00 \\
4 & 0.00 & 0.00 & 0.00 \\
5 & 0.00 & 0.00 & 0.00 \\
6 & 0.00 & 0.00 & 0.00 \\
7 & 0.00 & 0.00 & 0.00 \\
8 & 0.00 & 0.00 & 0.00 \\
9 & 0.00 & 0.01 & 0.00 \\
17 & 21 & 3.20 & 2.42 & 2.21 \\
10 & 0.01 & 0.01 & 0.01 & 14.11 & 10.52 & 9.75 \\
11 & 0.03 & 0.03 & 0.02 \\
12 & 0.07 & 0.06 & 0.05 \\
13 & 0.16 & 0.13 & 0.11 \\
14 & 0.43 & 0.27 & 0.23 & 127.18 & 21.50 & 20.49 \\
15 & 0.65 & 0.52 & 0.48 \\
16 & 1.48 & 1.13 & 1.02 \\
\hline
\end{tabular}

Table 3.2: Running time in seconds for several algorithms.

element shows an improvement in the GrayCodeNCD algorithm over the StandardNCD ranging from 26 and 35 percent for $20 \leq n \leq 30$. This mirrors the expected $28.6 \%$ decrease in run-time from the theoretical analysis.

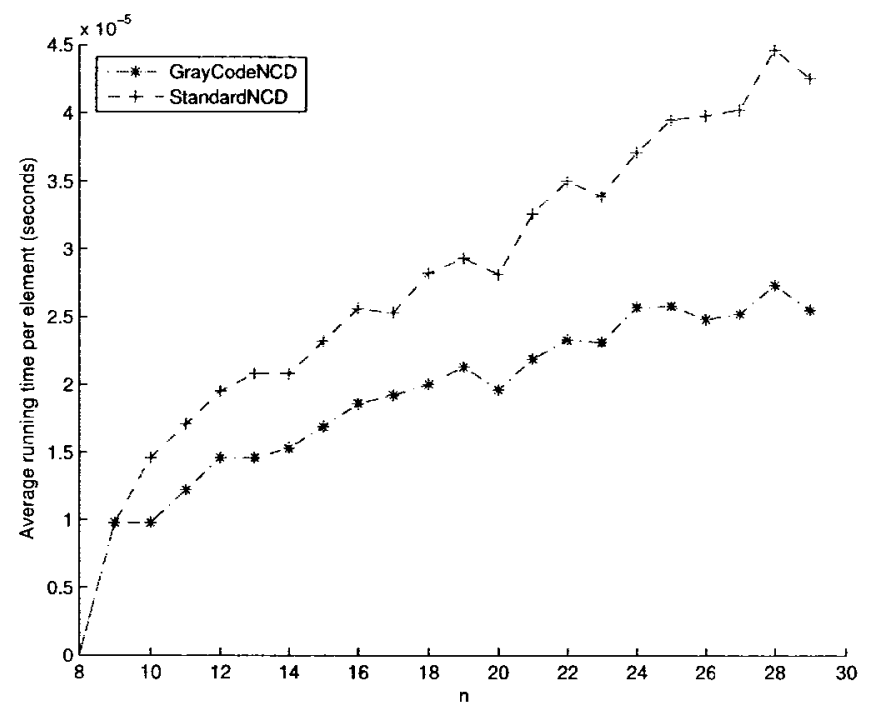

Figure 3.1: Average runtime per element for two exhaustive algorithms. 


\subsection{Conjectures}

To examine the distribution of normal bases of $\mathbb{F}_{2^{n}}$ over $\mathbb{F}_{2}$, in Figure 3.2 we plot the number of normal bases found against their complexity. In each case we observe a

(a)

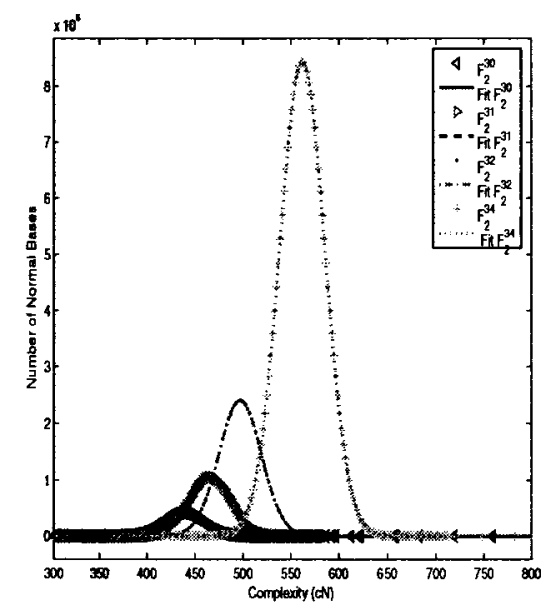

(b)

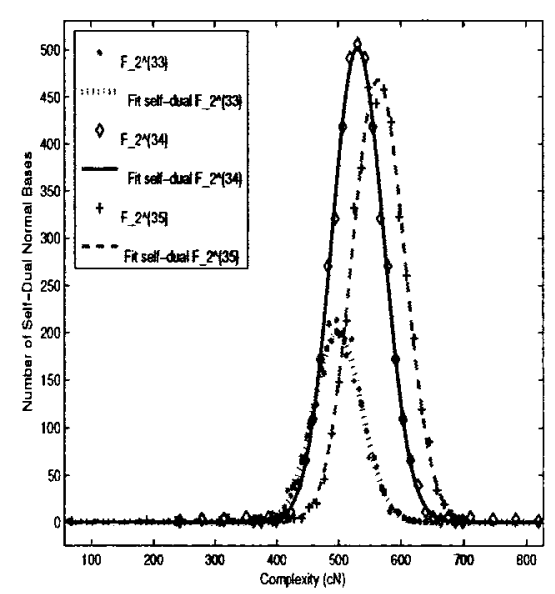

Figure 3.2: Sample distributions of complexity of (a) normal elements and (b) selfdual normal elements.

Gaussian shaped curve. This leads to the following conjecture.

Conjecture 3.3.1. The number of normal bases over $\mathbb{F}_{2^{n}}$ is normally distributed with respect to their complexities.

This conjecture is verified for the cases $n \leq 39$ by running the Shapiro-Wilk normality test [28] on the results of the GrayCodeNCD experiments, with over 99.9 percent certainty for $15 \leq n \leq 39$. Finding the general form of the distribution curve requires calculating the average complexity and the variance. As a consequence of this conjecture, although probabilistic algorithms to find normal elements exist [16], most normal elements exist close to the peak of the curve and these will not give 
low complexity normal elements. For efficient computation, new searching methods for normal elements must be developed. Alternatively, direct constructions of normal elements with low complexity can be explored, as in Chapter 4 .

Alternatively, an enveloping curve requires bounds (as functions of $n$ ) to find the averages and variances of the complexities. We observe that the probability of finding a normal element of complexity $c_{N} \leq k n$ is analogous to finding the density under the normalized curve as follows, $P\left(c_{N} \leq k n\right)=P[Z \leq(k n-\mu) / \sigma]$. A low complexity is analogous to a low Z-score on the normalized curve.

The data lends itself to the following conjecture on a bound for the average complexity of normal elements.

Conjecture 3.3.2. The average complexity of normal elements in a finite field $\mathbb{F}_{q^{n}}$ with $n \geq 8$ has an upper bound of $\left(n^{2}-n+3\right) / 2$.

This conjecture is based upon the data found using the GrayCodeNCD algorithm, and observing the symmetry of the Gaussian curve conjectured previously. This is very close to half of the largest possible complexity $n^{2}-n$, which is always even, so the extra factor of adding 3 in the numerator accounts for any decimal places calculated in the average. This conjectured bound is quite tight for many values of $n \leq 39$, and only for $n=14,23,25$ the numerator requires a constant term of 3 rather than 2 .

Conjecture 3.3.3. For sufficiently large $n$ the variance of complexities of normal bases is bounded above by $n^{2} / 2$.

This conjecture comes from the side-by-side comparison in Appendix B of the 
average and variances of the complexities. For this data $n \geq 19$ suffices. The variance and the average complexity are quite similar, which implies that there could probably be a stronger conjecture here, since this conjecture is loose in comparison to the one on the average complexity.

We recall that the average complexity of self-dual normal elements is given in Theorem 2.6.10, so the next step would naturally be to conjecture on the variance of the complexity of self-dual normal elements. A theoretical average for $n \equiv 2(\bmod 4)$ is given in Corollary 2.6.11, and our data agrees with this theory. However, since there are very few self-dual normal bases for $n \leq 36$ more experiments are required to give sufficient data to support any such conjecture.

The following argument leads to our final conjecture. Let us assume that the above conjectures on the average and variance of complexities of all normal elements in a finite field hold. Moreover, let us consider the following problem: is there some constant $k$ such that the minimum complexity element in a finite field $\mathbb{F}_{2^{n}}$ is bounded above by $k n$ ? Recall that an upper bound on the complexity of a normal element corresponds to a Z-score on the standardized normal curve. Given that by our conjectures $\mu \approx\left(n^{2}-n+3\right) / 2$ and $\sigma^{2} \approx n^{2} / 2$, calculating the Z-score of $k n$ gives

$$
z_{p}=\frac{k n-\frac{n^{2}-n+3}{2}}{n / \sqrt{2}}
$$

If $k$ is a constant, then, asymptotically, the Z-score becomes infinitely small. Relating this to the complexity distribution, this implies that the upper bound on the minimum 
complexity vanishes. Since the minimum possible complexity is $2 n-1$, this analysis leads to the following conclusion.

Conjecture 3.3.4. There is no constant $k$ such that the complexity $C_{n}$ of $\mathbb{F}_{2^{n}}$ is bounded above by $k n$ for all $n$. Furthermore, if the average and variance of the complexities of all normal elements in $\mathbb{F}_{2^{n}}$ are both of order $n^{2}$, then an upperbound for $C_{n}$, for all $n$, is also of order $n^{2}$.

Our final observation concerns the distribution of the normal elements themselves. To achieve results for $36 \leq n \leq 39$ we distributed the computation across many processors by letting each processor deal with a range of field elements that were consecutive in the Gray code order. This divided our time linearly with the number of processors used. For example, the estimated run time of $n=39$ was 8 months using a single processor, but we were able to complete the simulation in just under 3 weeks using 13 processors. Furthermore, we observed that there were large blocks along the run of the Gray code in which there were no normal elements found. This is an interesting topic for future research, which could lead to conclusions on the existence of normal elements with prescribed coefficients. 


\section{Chapter 4}

\section{Traces of Optimal Normal Bases}

As seen in Chapter 3 it is likely that the complexity of a random normal basis of $\mathbb{F}_{q^{n}}$ over $\mathbb{F}_{q}$ will be quite high: approximately $n^{2} / 2$ in the $q=2$ case. For this reason, constructions such as those given in Section 2.5 are required to find normal bases with low complexity. Ideally, these constructions should be easy to analyze and implement, and we would like the complexity of the basis to be close to $k n$ for some small value of $k$.

In this chapter we give constructions of low-complexity normal bases by projecting to subfields from fields containing both Type I and Type II optimal normal bases for all $q$. We do this by taking the trace function of the optimal normal element in the larger field. We also find the complexity of the dual basis of the bases obtained by these projection maps. A discussion of how large an extension must be to project down to a desired subfield is given, followed by a summary and comparison of these constructions to commonly used extensions. 


\subsection{The Trace of a Normal Element}

This chapter makes extensive use of the observation that the trace of a normal element is again normal. This is formally stated as the following proposition.

Proposition 4.1.1. Let $n=m k$ where $n, m, k$ are all positive integers, and let $\alpha$ be a normal element of $\mathbb{F}_{q^{n}}$ over $\mathbb{F}_{q}$. Then $\operatorname{Tr}_{q^{n} / q^{m}}(\alpha)$ is a normal element of $\mathbb{F}_{q^{m}}$ over $\mathbb{F}_{q}$

Proof. Define $n, \alpha \in \mathbb{F}_{q^{n}}$ as above, and let $\beta=\operatorname{Tr}_{q^{n} / q^{m}}(\alpha)$. Then

$$
\beta_{j}=\beta^{q^{j}}=\sum_{i=0}^{k-1} \alpha^{q^{m i+j}}, \quad 0 \leq j \leq m-1,
$$

and so for a fixed $j$ we have

$$
\sum_{i=0}^{k-1} c_{j} \alpha^{q^{m i+j}}=\left(\sum_{i=0}^{k-1} c_{j} \alpha^{q^{m i}}\right)^{q^{j}}
$$

for some $c_{j} \in \mathbb{F}_{q}$. The conjugates of $\alpha$ are linearly independent so setting Equation (4.1) equal to zero guarantees $c_{j}=0$ for all $0 \leq j \leq m-1$. Therefore the conjugates of $\beta$ are linearly independent and form a basis of $\mathbb{F}_{q^{m}}$ over $\mathbb{F}_{q}$.

\subsection{Main results}

We have seen that the trace of a normal element is again normal, so we use the trace function as a projection map from fields which contain an optimal normal basis to 
their subfields. We calculate upper bounds on the complexity of the basis generated by these projections for Type I and Type II Optimal normal bases, and for all $q$.

\subsubsection{Type I Optimal Normal Bases: $q$ odd}

Theorem 4.2.1. Let $\alpha \in \mathbb{F}_{q^{n}}$ generate an optimal normal basis of Type $I$ of $\mathbb{F}_{q^{n}}$ over $\mathbb{F}_{q}, q$ odd, and let $\beta=\operatorname{Tr}_{q^{n} / q^{m}}(\alpha) \in \mathbb{F}_{q^{m}}$ with $m=n / k$ and $k \leq m$. Then, the complexity of the normal basis of $\mathbb{F}_{q^{m}}$ over $\mathbb{F}_{q}$ generated by $\beta$ is bounded by $(k+$ 2) $m-3 k+1$, if $m$ is even and $k$ is odd and by $(k+1) m-k$ in all other cases.

Furthermore, for $1 \leq j \leq m-1$ row $j$ of the multiplication table of $\beta$ is a cyclic permutation of $j$ positions of row $(m-j)$.

Proof. Let $n+1$ be a prime, $q$ a primitive root modulo $n+1$ where $q$ is prime or prime power, $q \neq 2$ and $\alpha \in \mathbb{F}_{q^{n}}$ an optimal element of $\mathbb{F}_{q^{n}}$ over $\mathbb{F}_{q}$. Let $N=$ $\left\{\alpha, \alpha^{q}, \alpha^{q^{2}}, \ldots, \alpha^{q^{n-1}}\right\}$ be the optimal normal basis of $\mathbb{F}_{q^{n}}$ over $\mathbb{F}_{q}$ which is generated by $\alpha$.

The multiplication table $C_{[n \times n]}$ of the linear map

$$
C_{\alpha}: \mathbb{F}_{q^{n}} \rightarrow \mathbb{F}_{q^{n}}, \quad C_{\alpha}(x)=\alpha \cdot x
$$

has exactly $2 n-1$ non-zero terms with the following properties:

$$
\alpha \cdot \alpha^{q^{i}}=\alpha^{q^{j}}, \quad i=0,1, \ldots, \frac{n}{2}-1, \frac{n}{2}+1, \ldots, n-1, \quad j=0,1, \ldots, n-1,
$$




$$
\alpha \cdot \alpha^{q^{n / 2}}=\sum_{s=0}^{n-1}-\alpha^{q^{s}}
$$

The above equations imply that there is exactly one 1 in each row except for the row $n / 2$, when $n$ is even, where all the $n$ entries are -1 .

Suppose that $\beta=\operatorname{Tr}_{q^{n} / q^{m}}(\alpha) \in \mathbb{F}_{q^{m}}$ with $m=n / k$. Then,

$$
\beta=\operatorname{Tr}_{q^{n} / q^{m}}(\alpha)=\sum_{i=0}^{k-1} \alpha^{q^{m i}}=\alpha+\alpha^{q^{m}}+\alpha^{q^{2 m}}+\cdots+\alpha^{q^{(k-1) m}}
$$

generates a normal basis $M$ of $\mathbb{F}_{q^{m}}$ over $\mathbb{F}_{q}$ of the form

$$
M=\left\{\beta, \beta^{q}, \beta^{q^{2}}, \ldots, \beta^{q^{m-1}}\right\}
$$

We observe that, for $j=0, \ldots, m-1$, we have

$$
\beta^{q^{j}}=\sum_{i=0}^{k-1} \alpha^{q^{m i+j}}=\alpha^{q^{j}}+\alpha^{q^{j+m}}+\cdots+\alpha^{q^{j+(k-1) m}}
$$

Let $D=D_{[m \times m]}$ be the multiplication table of the linear map

$$
D_{\beta}: \mathbb{F}_{q^{m}} \rightarrow \mathbb{F}_{q^{m}}, \quad D_{\beta}(x)=\beta \cdot x
$$

The first row of the table $D$ is given by

$$
\beta \cdot \beta=\left(\sum_{i=0}^{k-1} \alpha^{q^{m i}}\right) \cdot\left(\sum_{i=0}^{k-1} \alpha^{q^{m i}}\right)
$$




$$
\begin{aligned}
= & \sum_{i=0}^{k-1}(\alpha \cdot \alpha)^{q^{m i}}+\sum_{i=0}^{k-1}\left(\alpha \cdot \alpha^{q^{m}}\right)^{q^{m i}}+\cdots+\sum_{i=0}^{k-1}\left(\alpha \cdot \alpha^{q^{(k m) / 2}}\right)^{q^{m i}} \\
& +\cdots+\sum_{i=0}^{k-1}\left(\alpha \cdot \alpha^{q^{(k-1) m}}\right)^{q^{m i}} .
\end{aligned}
$$

Using Equations (4.2) and (4.3), there are $\mu_{0}, \mu_{1}, \ldots, \mu_{k-2} \in \mathbb{Z}_{n}$ such that

$$
\alpha \cdot \alpha=\alpha^{q^{\mu_{0}}}, \alpha \cdot \alpha^{q^{m}}=\alpha^{q^{\mu_{1}}}, \ldots, \alpha \cdot \alpha^{q^{(k-1) m}}=\alpha^{q^{\mu_{k-2}}}
$$

and

$$
\sum_{i=0}^{k-1}\left(\alpha \cdot \alpha^{q^{n / 2}}\right)^{q^{m i}}=\sum_{i=0}^{k-1}\left(-\sum_{s=0}^{n-1} \alpha^{q^{s}}\right)^{q^{m i}}=\sum_{i=0}^{k-1}\left(-\sum_{s=0}^{m-1} \beta^{q^{s}}\right)^{q^{m i}}=-k \sum_{s=0}^{m-1} \beta^{q^{s}}
$$

Thus, we get

$$
\begin{aligned}
\beta \cdot \beta= & \sum_{i=0}^{k-1}\left(\alpha^{q^{\mu_{0}}}\right)^{q^{m i}}+\sum_{i=0}^{k-1}\left(\alpha^{q^{\mu_{1}}}\right)^{q^{m i}}+\cdots+\sum_{i=0}^{k-1}\left(\alpha^{q^{\mu_{k-2}}}\right)^{q^{m i}}-k \sum_{s=0}^{m-1} \beta^{q^{s}} \\
= & \beta^{q^{\mu_{0}}}+\beta^{q^{\mu_{1}}}+\cdots+\beta^{q^{\mu_{k-2}}}-k\left(\beta+\beta^{q}+\cdots+\beta^{q^{m-1}}\right) \\
= & -k \beta-k \beta^{q}+\cdots+(1-k) \beta^{q^{\mu_{0}}}+(1-k) \beta^{q^{\mu_{1}}}+\cdots+(1-k) \beta^{q^{\mu_{k-2}}} \\
& +\cdots+(-k) \beta^{q^{m-1}}
\end{aligned}
$$

The coefficients of $\beta^{q^{j}}$ are computed modulo $q$, so the first row of the table has at most $m$ non-zero terms. 
Then, we calculate the remaining rows $j=1, \ldots, m-1$ of the table by computing

$$
\begin{aligned}
\beta \cdot \beta^{q^{j}} & =\left(\sum_{i=0}^{k-1} \alpha^{q^{m i}}\right) \cdot\left(\sum_{u=0}^{k-1} \alpha^{q^{m u+j}}\right) \\
& =\sum_{0 \leq u, i \leq k-1}\left(\alpha^{q^{m i}}\right)\left(\alpha^{q^{m u+j}}\right) \\
& =\sum_{i=0}^{k-1}\left(\alpha \cdot \alpha^{q^{j}}\right)^{q^{i m}}+\sum_{i=0}^{k-1}\left(\alpha \cdot \alpha^{q^{j+m}}\right)^{q^{i m}}+\cdots+\sum_{i=0}^{k-1}\left(\alpha \cdot \alpha^{q^{j+(k-1) m}}\right)^{q^{i m}} .
\end{aligned}
$$

By Equation (4.2) there are $\lambda_{0}, \lambda_{1}, \ldots, \lambda_{k-1} \in \mathbb{Z}_{n}$ such that

$$
\alpha \cdot \alpha^{q^{j}}=\alpha^{q^{\lambda_{0}}}, \quad \alpha \cdot \alpha^{q^{j+m}}=\alpha^{q^{\lambda_{1}}}, \ldots, \alpha \cdot \alpha^{q^{j+(k-1) m}}=\alpha^{q^{\lambda_{k-1}}}
$$

which implies,

$$
\begin{aligned}
\beta \cdot \beta^{q^{j}} & =\sum_{i=0}^{k-1}\left(\alpha^{q^{\lambda_{0}}}\right)^{q^{i m}}+\sum_{i=0}^{k-1}\left(\alpha^{q^{\lambda_{1}}}\right)^{q^{i m}}+\cdots+\sum_{i=0}^{k-1}\left(\alpha^{q^{\lambda_{k-1}}}\right)^{q^{i m}} \\
& =\beta^{q^{\lambda_{0}}}+\beta^{q^{\lambda_{1}}}+\cdots+\beta^{q^{\lambda_{k-1}}}
\end{aligned}
$$

Finally, for the row $(m-j)$ of the table $D$, we have

$$
\beta \cdot \beta^{q^{m-j}}=\sum_{i=0}^{k-1}\left(\alpha \cdot \alpha^{q^{m-j}}\right)^{q^{i m}}+\sum_{i=0}^{k-1}\left(\alpha \cdot \alpha^{q^{2 m-j}}\right)^{q^{i m}}+\cdots+\sum_{i=0}^{k-1}\left(\alpha \cdot \alpha^{q^{-j}}\right)^{q^{i m}}
$$

Using the identities (4.4) it follows that

$$
\beta \cdot \beta^{q^{m-j}}=\beta^{q^{\lambda_{k-1}+m-j}}+\beta^{q^{\lambda_{k-2}+2 m-j}}+\cdots+\beta^{q^{\lambda_{0}-j}}
$$


and since $\beta^{q^{m}}=\beta$ we get

$$
\beta \cdot \beta^{q^{m-j}}=\beta^{q^{\lambda_{k-1}-j}}+\beta^{q^{\lambda_{k-2}-j}}+\cdots+\beta^{q^{\lambda_{0}-j}} .
$$

Thus, the row $j$ of the multiplication table of $\beta$ is a cyclic permutation of $j$ positions of row $(m-j)$.

If $m=n / k$ is an even number, to calculate the row $m / 2$ of the table $D$ we must consider both the cases where $k$ is even and where $k$ is odd. For the odd case we have

$$
\begin{aligned}
\beta \cdot \beta^{q^{m / 2}}= & \left(\sum_{i=0}^{k-1} \alpha^{q^{m i}}\right) \cdot\left(\sum_{u=0}^{k-1} \alpha^{q^{m u+m / 2}}\right) \\
= & \sum_{0 \leq u, i \leq k-1}\left(\alpha^{q^{m i}}\right)\left(\alpha^{q^{m u+m / 2}}\right) \\
= & \sum_{i=0}^{k-1}\left(\alpha \cdot \alpha^{q^{m / 2}}\right)^{q^{i m}}+\sum_{i=0}^{k-1}\left(\alpha \cdot \alpha^{q^{m / 2+m}}\right)^{q^{i m}}+\cdots \\
& +\sum_{i=0}^{k-1}\left(\alpha \cdot \alpha^{q^{m / 2+m(k-1) / 2}}\right)^{q^{i m}}+\cdots+\sum_{i=0}^{k-1}\left(\alpha \cdot \alpha^{q^{m / 2+(k-1) m}}\right)^{q^{i m}} \\
= & \sum_{i=0}^{k-1}\left(\alpha \cdot \alpha^{q^{m / 2}}\right)^{q^{i m}}+\sum_{i=0}^{k-1}\left(\alpha \cdot \alpha^{q^{m / 2+m}}\right)^{q^{i m}}+\cdots \\
& +\sum_{i=0}^{k-1}\left(\alpha \cdot \alpha^{q^{n / 2}}\right)^{q^{i m}}+\cdots+\sum_{i=0}^{k-1}\left(\alpha \cdot \alpha^{q^{m / 2+(k-1) m}}\right)^{q^{i m}} .
\end{aligned}
$$

By Equations (4.2) and (4.3), there are $\delta_{0}, \delta_{1}, \cdots, \delta_{k-2} \in \mathbb{Z}_{n}$ such that

$$
\beta \cdot \beta^{q^{m / 2}}=\beta^{q^{\delta_{0}}}+\beta^{q^{\delta_{1}}}+\cdots+\left(-\beta-\beta^{q}-\beta^{q^{2}}-\cdots-\beta^{q^{m-1}}\right)+\cdots+\beta^{g^{\delta_{k-2}}} .
$$

Thus, the row $m / 2$ in this case has at most $m-k+1$ non-zero terms. For the case 
where $k$ is even, the computations of (4.5) are similar to the calculations involving the identities (4.4) above, and yield at most $k$ non-zero terms.

In conclusion, we observe that an upper bound for the complexity of the normal basis of $\mathbb{F}_{q^{m}}$ over $\mathbb{F}_{q}$ generated by $\beta$, when $m$ is even and $k$ is odd is $k(m-2)+2 m-$ $k+1=(k+2) m-3 k+1$ since the first row of the table gives at most $m$ non-zero entries, the $m / 2$ row gives at most $m-k+1$ entries and all other rows give at most $k$ entries. In all other cases, the upper bound for the complexity of the normal basis is $k(m-1)+m=(k+1) m-k$, since the first row gives at most $m$ non-zero terms and all other rows give at most $k$ non-zero terms.

\subsubsection{Type I Optimal Normal Bases: $q$ even}

Theorem 4.2.2. Let $\alpha \in \mathbb{F}_{2^{n}}$ generate an optimal normal basis of Type $I$ of $\mathbb{F}_{2^{n}}$ over $\mathbb{F}_{2}, n>2$, and let $\beta=\operatorname{Tr}_{2^{n} / 2^{m}}(\alpha) \in \mathbb{F}_{2^{n}}$ with $m=n / k$ and $k \leq m$. Then, an upper bound for the complexity of the normal basis of $\mathbb{F}_{2^{m}}$ over $\mathbb{F}_{2}$ generated by $\beta$ is $(k+1) m-3 k+2$ if $m$ is even and $k$ is odd, or $k m-k+1$ otherwise.

Furthermore, for $1 \leq j \leq m-1$ row $j$ of the multiplication table of $\beta$ is a cyclic permutation of $j$ positions of row $(m-j)$.

Proof. We observe that the proof of this claim is nearly identical to the case where $q$ is odd except for the following changes.

Let $n+1$ be a prime, 2 a primitive root modulo $n+1$, and let $\alpha \in \mathbb{F}_{2^{n}}$ be an optimal

element of $\mathbb{F}_{2^{n}}$ over $\mathbb{F}_{2}$. Let $N=\left\{\alpha, \alpha^{2}, \alpha^{2^{2}}, \ldots, \alpha^{2^{n-1}}\right\}$ be the optimal normal basis 
of $\mathbb{F}_{2^{n}}$ over $\mathbb{F}_{2}$ which is generated by $\alpha$.

The multiplication table $C_{[n \times n]}$ of the linear map

$$
C_{\alpha}: \mathbb{F}_{2^{n}} \rightarrow \mathbb{F}_{2^{n}}, \quad C_{\alpha}(x)=\alpha \cdot x
$$

has exactly $2 n-1$ non-zero terms with the following properties:

$$
\begin{gathered}
\alpha \cdot \alpha^{2^{i}}=\alpha^{2^{j}}, \quad i=0,1, \ldots, \frac{n}{2}-1, \frac{n}{2}+1, \ldots, n-1, \quad j=0,1, \ldots, n-1, \\
\alpha \cdot \alpha^{2^{n / 2}}=\sum_{s=0}^{n-1}-\alpha^{2^{s}}=\sum_{s=0}^{n-1} \alpha^{2^{s}} .
\end{gathered}
$$

The above equations imply that there is exactly one 1 in each row except for the row $n / 2$ where all the $n$ entries are 1.

Suppose that $\beta=\operatorname{Tr}_{2^{n} / 2^{m}}(\alpha) \in \mathbb{F}_{2^{m}}$ with $m=n / k$. Then,

$$
\beta=\operatorname{Tr}_{2^{n} / 2^{m}}(\alpha)=\sum_{i=0}^{k-1} \alpha^{2^{m i}}=\alpha+\alpha^{2^{m}}+\alpha^{2^{2 m}}+\cdots+\alpha^{2^{(k-1) m}}
$$

generates a normal basis $M$ of $\mathbb{F}_{2^{m}}$ over $\mathbb{F}_{2}$ of the form

$$
M=\left\{\beta, \beta^{2}, \beta^{2^{2}}, \ldots, \beta^{2^{m-1}}\right\}
$$


We have that, for $j=0, \ldots, m-1$,

$$
\beta^{2^{j}}=\sum_{i=0}^{k-1} \alpha^{2^{m i+j}}=\alpha^{2^{j}}+\alpha^{2^{j+m}}+\cdots+\alpha^{2^{j+(k-1) m}}
$$

Let $D=D_{[m \times m]}$ be the multiplication table of the linear map

$$
D_{\beta}: \mathbb{F}_{2^{m}} \rightarrow \mathbb{F}_{2^{m}}, \quad D_{\beta}(x)=\beta \cdot x
$$

The first row of the table $D$ is given by $\beta \cdot \beta=\beta^{2}$. Thus, the first row of the table $D$ has a 1 in the second position.

If $m=n / k$ is an even number then for the row $m / 2$ of the table, by Equation (4.5), we have that this row contributes at most $m-k+1$ ones to $D$ if $k$ is odd, and at most $k$ ones to $D$ if $k$ is even.

For the remaining rows the proof is identical to the $q$ odd case. We recall that each of the remaining rows contribute to the complexity with at most $k$ non-zero entries. The proof that row $j$ of the multiplication of $\beta$ is a cyclic permutation of $j$ positions of row $(m-j)$ is also identical to the $q$ odd case.

In conclusion, we observe that an upper bound for the complexity of the normal basis of $\mathbb{F}_{2^{m}}$ over $\mathbb{F}_{2}$ generated by $\beta$, when $m$ is odd or if both $m$ and $k$ are even, is $k(m-1)+1=k m-k+1$. Otherwise, if $m$ is even and $k$ is odd, the complexity is at most $k(m-2)+m-k+2=(k+1) m-3 k+2$. 


\subsubsection{Type II Optimal Normal Bases}

Theorem 4.2.3. Let $\alpha$ generate a Type II optimal normal basis of $\mathbb{F}_{2^{n}}$ over $\mathbb{F}_{2}$ and let $\beta=\operatorname{Tr}_{2^{n} / 2^{m}}(\alpha) \in \mathbb{F}_{2^{m}}$ with $m=n / k$ and $k \leq m$. Then the complexity of the normal basis of $\mathbb{F}_{2^{m}}$ over $\mathbb{F}_{2}$ generated by $\beta$ is $2 k m-2 k+1$.

Proof. Let $2 n+1$ be prime, and suppose that either

1. 2 is a primitive element of $\mathbb{Z}_{2 n+1}$, or

2. $2 n+1 \equiv 3(\bmod 4)$ and 2 generates the quadratic residues in $\mathbb{Z}_{2 n+1}$.

Let $\alpha=\gamma+\gamma^{-1}$, where $\gamma$ is a primitive $(2 n+1)$ th root of unity. Let $N=$ $\left\{\alpha, \alpha^{2}, \alpha^{2^{2}}, \ldots\right.$,

$\left.\alpha^{2^{n-1}}\right\}$ be the optimal normal basis of $\mathbb{F}_{2^{n}}$ over $\mathbb{F}_{2}$ which is generated by $\alpha$.

The multiplication table $C_{[n \times n]}$ of the linear map

$$
C_{\alpha}: \mathbb{F}_{2^{n}} \rightarrow \mathbb{F}_{2^{n}}, \quad C_{\alpha}(x)=\alpha \cdot x
$$

has exactly $2 n-1$ non-zero terms with the following property:

$$
\alpha \cdot \alpha^{2^{i}}=\alpha^{2^{j}}+\alpha^{2^{k}}, \quad i=1, \ldots, n-1, \quad j, k=0,1, \ldots, n-1, j \neq k
$$

Therefore, there are exactly two ones in each row of $C$ except for the first row, where there is one 1 in the second position. 
Suppose that $\beta=\operatorname{Tr}_{2^{n} / 2^{m}}(\alpha) \in \mathbb{F}_{2^{m}}$ with $m=n / k$. Then,

$$
\beta=\operatorname{Tr}_{2^{n} / 2^{m}}(\alpha)=\sum_{i=0}^{k-1} \alpha^{2^{m i}}=\alpha+\alpha^{2^{m}}+\alpha^{2^{2 m}}+\cdots+\alpha^{2^{(k-1) m}}
$$

generates a normal basis $M$ of $\mathbb{F}_{2^{m}}$ over $\mathbb{F}_{2}$ of the form

$$
M=\left\{\beta, \beta^{2}, \beta^{2^{2}}, \ldots, \beta^{2^{m-1}}\right\}
$$

We observe that, for $j=0, \ldots, m-1$, we have

$$
\beta^{2^{j}}=\sum_{i=0}^{k-1} \alpha^{2^{j+m i}}=\alpha^{2^{j}}+\alpha^{2^{j+m}}+\cdots+\alpha^{2^{j+(k-1) m}}
$$

Let $D=D_{[m \times m]}$ be the multiplication table of the linear map

$$
D_{\beta}: \mathbb{F}_{2^{m}} \rightarrow \mathbb{F}_{2^{m}}, \quad D_{\beta}(x)=\beta \cdot x
$$

The first row of the table $D$ is given by $\beta \cdot \beta=\beta^{2}$. Thus, it has 1 non-zero term in the second position.

As in Theorem 4.2.2, computing the $j$ th row of $D$ gives

$$
\beta \cdot \beta^{2^{j}}=\sum_{i=0}^{k-1}\left(\alpha \cdot \alpha^{2^{j}}\right)^{2^{m i}}+\sum_{i=0}^{k-1}\left(\alpha \cdot \alpha^{2^{j+m}}\right)^{2^{m i}}+\cdots+\sum_{i=0}^{k-1}\left(\alpha \cdot \alpha^{2^{j+(k-1) m}}\right)^{2^{m i}}
$$

By Equation (4.8), there exist $\lambda_{i}, \mu_{i} \in \mathbb{Z}_{n}$ such that $\alpha \cdot \alpha^{2^{j}+m i}=\alpha^{2^{\lambda_{i}}}+\alpha^{2^{\mu_{i}}}, i=$ 
$0, \ldots, k-1$. So

$$
\begin{aligned}
\beta \cdot \beta^{2^{j}} & =\sum_{i=0}^{k-1}\left(\alpha^{2^{\lambda_{0}}}+\alpha^{2^{\mu_{0}}}\right)^{2^{m i}}+\cdots+\sum_{i=0}^{k-1}\left(\alpha^{2^{\lambda_{k-1}}}+\alpha^{2^{\mu_{k-1}}}\right)^{2^{m i}} \\
& =\beta^{2^{\lambda_{0}}}+\beta^{2^{\mu_{0}}}+\cdots+\beta^{2^{\lambda_{k-1}}}+\beta^{2^{\mu_{k-1}}} .
\end{aligned}
$$

Thus, there are $2 k$ ones appearing in each remaining row of $D$.

In conclusion, we observe that the complexity of the basis generated by $\beta \in \mathbb{F}_{2^{m}}$ over $\mathbb{F}_{2}$ is $2 k(m-1)+1$.

The following corollary shows that, in contrast with the case Type I normal basis and $q$ even, we do not obtain optimal normal elements for Type II normal basis. Hence, this corollary gives new low complexity normal elements for $q$ even.

Corollary 4.2.4. Let $\alpha$ generate a Type II optimal normal basis of $\mathbb{F}_{2^{n}}$ over $\mathbb{F}_{2}$ and let $\beta=\operatorname{Tr}_{2^{n} / 2^{m}}(\alpha) \in \mathbb{F}_{2^{m}}$ with $m=n / 2$. Then the complexity of the normal basis of $\mathbb{F}_{2^{m}}$ over $\mathbb{F}_{2}$ generated by $\beta$ is $4 m-3$.

\subsection{The Dual of the Trace of an Optimal Element}

Let $N=\left\{\alpha, \alpha_{1}, \ldots, \alpha_{n-1}\right\}$ be a normal basis of $\mathbb{F}_{q^{n}}$ over $\mathbb{F}_{q}$ and $M=\left\{\gamma, \gamma_{1}, \ldots, \gamma_{n-1}\right\}$ be another basis. Then $M$ is the dual basis of $N$ if $\operatorname{Tr}\left(\alpha_{i} \gamma_{j}\right)=\delta_{i j}$ for $1 \leq i, j \leq n-1$ where $\delta_{i j}$ is the Kronecker delta function. Proposition 4.1.1 states that the dual basis of a normal basis is again normal, so let $\gamma_{i}=\gamma^{q^{i}}$. Theorem 4.3.1 gives the statement and proof of an upper bound of the complexity of the dual basis of the trace of a Type 
I optimal normal basis when $q$ is odd. Following the proof we present a statement of the theorem using Type II optimal normal bases and a summary table outlining upper bounds for any $q$ and for both Type I and Type II optimal normal bases.

Theorem 4.3.1. Let $\alpha \in \mathbb{F}_{q^{n}}$ generate an optimal normal basis of Type I of $\mathbb{F}_{q^{n}}$ over $\mathbb{F}_{q}$ and let $\beta=\operatorname{Tr}_{q^{n}} / q^{m}(\alpha) \in \mathbb{F}_{q^{m}}$ with $m=n / k, k \leq m$. Then the complexity of the normal basis of $\mathbb{F}_{q^{m}}$ over $\mathbb{F}_{q}$ generated by $\gamma$, which is the dual element of $\beta$, is $(k+2) m-2$ when $m$ is odd. Further, for $1 \leq j \leq m-1$ row $j$ of the multiplication table of $\gamma$ is a cyclic permutation of $j$ positions of row $(m-j)$.

Proof. As in Theorem 4.2.1, let $\alpha \in \mathbb{F}_{q^{n}}$ generate an optimal normal basis of Type I and $\beta=\operatorname{Tr}_{q^{n} / q^{m}}(\alpha) \in \mathbb{F}_{q^{m}}$ with $m=n / k$. Let $\gamma \in \mathbb{F}_{q^{m}}$ be a dual element of $\beta$. According to Theorem 2.6.6, $\gamma$ is of the form

$$
\gamma=d_{0} \beta+d_{1} \beta^{q}+\cdots+d_{m-1} \beta^{q^{m-1}}
$$

where $d_{0}, d_{1}, \ldots, d_{m-1} \in \mathbb{F}_{q}$ are the coefficients of the unique polynomial $g(x)$ of degree $\leq m-1$ satisfying

$$
g(x) h(x) \equiv 1 \quad\left(\bmod x^{m}-1\right)
$$

and $h(x)$ is of degree $\leq m-1$ with coefficients $t_{0}, t_{1}, \ldots, t_{m-1}$ where,

$$
t_{i}=\operatorname{Tr}_{q^{m} / q}\left(\beta \cdot \beta^{q^{i}}\right), \quad i=0,1, \ldots, m-1
$$


Since, $\operatorname{Tr}_{q^{m} / q}(\beta) \cdot \operatorname{Tr}_{q^{m} / q}(\gamma)=1$ and $\operatorname{Tr}_{q^{m} / q}(\beta)=-1$ we get,

$$
d_{0}+d_{1}+\cdots+d_{m-1}=1
$$

We compute $t_{0}=\operatorname{Tr}_{q^{m} / q}(\beta \cdot \beta)$ separate from $t_{i}=\operatorname{Tr}_{q^{m} / q}\left(\beta \cdot \beta^{q^{i}}\right), i=1, \ldots, m-1$.

$$
\begin{aligned}
t_{0}= & \operatorname{Tr}_{q^{n / k} / q}(\beta \cdot \beta)=\frac{1}{k} \operatorname{Tr}_{q^{n} / q}(\beta \cdot \beta)=\frac{1}{k} \operatorname{Tr}_{q^{n} / q}\left(\left(\sum_{i=0}^{k-1} \alpha^{q^{m i}}\right)\left(\sum_{i=0}^{k-1} \alpha^{q^{m i}}\right)\right) \\
= & \frac{1}{k} \operatorname{Tr}_{q^{n} / q}\left(\sum_{i=0}^{k-1}(\alpha \cdot \alpha)^{q^{m i}}+\sum_{i=0}^{k-1}\left(\alpha \cdot \alpha^{q^{m}}\right)^{q^{m i}}+\cdots+\sum_{i=0}^{k-1}\left(\alpha \cdot \alpha^{q^{(k m) / 2}}\right)^{q^{m i}}+\right. \\
& \left.+\cdots+\sum_{i=0}^{k-1}\left(\alpha \cdot \alpha^{q^{(k-1) m}}\right)^{q^{m i}}\right)
\end{aligned}
$$

using Equations (4.2),(4.3) there are $\mu_{0}, \mu_{1}, \ldots, \mu_{k-1}, \in \mathbb{Z}_{m}$ such that

$$
\alpha \cdot \alpha=\alpha^{q^{\mu_{0}}}, \alpha \cdot \alpha^{q^{m}}=\alpha^{q^{\mu_{1}}}, \ldots, \alpha \cdot \alpha^{q^{(k-1) m}}=\alpha^{q^{\mu_{k-2}}}
$$

Thus

$$
\begin{aligned}
t_{0}= & \frac{1}{k} \operatorname{Tr}_{q^{n} / q}\left(\sum_{i=0}^{k-1}\left(\alpha^{q^{\mu_{0}}}\right)^{q^{m i}}+\sum_{i=0}^{k-1}\left(\alpha^{q^{\mu_{1}}}\right)^{q^{m i}}+\cdots+k+\cdots+\sum_{i=0}^{k-1}\left(\alpha^{\left.\left.q^{\mu_{k-2}}\right)^{q^{m i}}\right)}\right.\right. \\
= & \frac{1}{k}\left(\sum_{i=0}^{k-1} \operatorname{Tr}_{q^{n} / q}\left(\alpha^{q^{\mu_{0}}}\right)^{q^{m i}}+\sum_{i=0}^{k-1} \operatorname{Tr}_{q^{n} / q}\left(\alpha^{q^{\mu_{1}}}\right)^{q^{m i}}+\cdots+\operatorname{Tr}_{q^{n} / q}(k)+\cdots+\right. \\
& \left.+\cdots+\sum_{i=0}^{k-1} \operatorname{Tr}_{q^{n} / q}\left(\alpha^{q^{\mu_{k-2}}}\right)^{q^{m i}}\right) \\
= & \frac{1}{k}((-k)+(-k)+\cdots+k n+\cdots+(-k)) \\
= & \left.\frac{1}{k}((-k)(k-1)+k n)\right)=n-k+1 .
\end{aligned}
$$


Now, we calculate $t_{i}=\operatorname{Tr}_{q^{m} / q}\left(\beta \cdot \beta^{q^{i}}\right), \quad i=1, \ldots, m-1$. By Theorem 4.2.1, there are $\lambda_{0}, \lambda_{1}, \ldots, \lambda_{k-1} \in \mathbb{Z}_{m}$ such that

$$
\begin{gathered}
\beta \cdot \beta^{q^{i}}=\beta^{q^{\lambda_{0}}}+\beta^{q^{\lambda_{1}}}+\cdots+\beta^{q^{\lambda_{k-1}}} . \\
t_{i}=\operatorname{Tr}_{q^{m} / q}\left(\beta \cdot \beta^{q^{i}}\right)=\operatorname{Tr}_{q^{m} / q}\left(\beta^{q^{\lambda_{0}}}+\beta^{q^{\lambda_{1}}}+\cdots+\beta^{q^{\lambda_{k-1}}}\right) \\
=\operatorname{Tr}_{q^{m} / q}\left(\beta^{q^{\lambda_{0}}}\right)+\operatorname{Tr}_{q^{m} / q}\left(\beta^{q^{\lambda_{1}}}\right)+\cdots+\operatorname{Tr}_{q^{m} / q}\left(\beta^{q^{\lambda_{k-1}}}\right) \\
=(-1)+(-1)+\cdots+(-1)=-k .
\end{gathered}
$$

This implies,

$$
h(x)=-k\left(x^{m-1}+x^{m-2}+\cdots+x\right)+n-k+1 .
$$

As in Theorem 2.6.6, we compute the $d_{i}$ coefficients by the system

$$
\begin{aligned}
& d_{0} t_{0}+d_{1} t_{-1}+\cdots+d_{m-1} t_{-(m-1)}=1 \\
& d_{0} t_{1}+d_{1} t_{0}+\cdots+d_{m-1} t_{-(m-2)}=0 \\
& \begin{array}{llll}
\vdots & \vdots & \vdots & \vdots
\end{array} \\
& d_{0} t_{m-2}+d_{1} t_{m-1}+\cdots+d_{m-1} t_{-1}=0 \\
& d_{0} t_{m-1}+d_{1} t_{m-2}+\cdots+d_{m-1} t_{0}=0 .
\end{aligned}
$$

The indices of $t_{i}$ are computed $(\bmod m)$ and the $d_{i} \in \mathbb{F}_{q}$ are found by solving the 
system

$$
\left(\begin{array}{ccccc}
n-k+1 & -k & \ldots & -k & -k \\
-k & n-k+1 & \ldots & -k & -k \\
\vdots & \ldots & \ldots & \vdots & \vdots \\
-k & -k & \ldots & n-k+1 & -k \\
-k & -k & \ldots & -k & n-k+1
\end{array}\right)\left(\begin{array}{c}
d_{0} \\
d_{1} \\
\vdots \\
d_{m-2} \\
d_{m-1}
\end{array}\right)=\left(\begin{array}{c}
1 \\
0 \\
\vdots \\
0 \\
0
\end{array}\right)
$$

which implies that

$$
d_{0}=\frac{k+1}{n+1}, \quad d_{i}=\frac{k}{n+1}, \quad i=1, \ldots, m-1
$$

Note that $n+1$ is a prime different from zero in $\mathbb{F}_{q}$, and therefore has an inverse.

Then using that $\operatorname{Tr}_{q^{m} / q}(\beta)=-1$ the dual element $\gamma \in \mathbb{F}_{q^{m}}$ is :

$$
\begin{aligned}
\gamma & =\frac{k+1}{n+1} \beta+\frac{k}{n+1}\left(\beta^{q}+\beta^{q^{2}}+\cdots+\beta^{q^{m-1}}\right) \\
& =\frac{1}{n+1} \beta+\frac{k}{n+1}\left(\beta+\beta^{q}+\beta^{q^{2}}+\cdots+\beta^{q^{m-1}}\right) \\
& =\frac{1}{n+1} \beta+\frac{k}{n+1} \operatorname{Tr}_{q^{m} / q}(\beta) \\
& =\frac{1}{n+1} \beta-\frac{k}{n+1}
\end{aligned}
$$

and $\gamma^{q^{i}}=\frac{1}{n+1} \beta^{q^{2}}-\frac{k}{n+1}, \quad i=0, \ldots, m-1$. 
Let $C=C_{[m \times m]}$ be the multiplication table of the linear map

$$
C_{\gamma}: \mathbb{F}_{q^{m}} \rightarrow \mathbb{F}_{q^{m}}, \quad C_{\gamma}(x)=\gamma \cdot x
$$

By Theorem 4.2.1, there exist $\mu_{0}, \mu_{1}, \ldots, \mu_{k-2} \in \mathbb{Z}_{m}$ such that

$$
\beta \cdot \beta=\beta^{q^{\mu_{0}}}+\beta^{q^{\mu_{1}}}+\cdots+\beta^{q^{\mu_{k-2}}}-k\left(\beta+\beta^{q}+\cdots+\beta^{q^{m-1}}\right) .
$$

The first row of the table $C$ is given by:

$$
\begin{aligned}
\gamma \cdot \gamma= & \left(\frac{1}{n+1} \beta-\frac{k}{n+1}\right) \cdot\left(\frac{1}{n+1} \beta-\frac{k}{n+1}\right) \\
= & \frac{1}{(n+1)^{2}} \beta \cdot \beta-\frac{2 k}{(n+1)^{2}} \beta+\frac{k^{2}}{(n+1)^{2}} \\
= & \frac{1}{(n+1)}\left(\left(\frac{1}{n+1} \beta^{q^{\mu_{0}}}-\frac{k}{n+1}\right)+\cdots+\left(\frac{1}{n+1} \beta^{q^{\mu_{k-2}}}-\frac{k}{n+1}\right)\right. \\
& \left.-k \operatorname{Tr}_{q^{m} / q}(\beta)\right)+\frac{k(k-1)}{(n+1)^{2}}-\frac{2 k}{(n+1)^{2}} \beta+\frac{k^{2}}{(n+1)^{2}} \\
= & \frac{1}{(n+1)}\left(\gamma^{q^{\mu_{0}}}+\gamma^{q^{\mu_{1}}}+\cdots+\gamma^{q^{\mu_{k-2}}}\right)-\frac{2 k}{n+1} \gamma
\end{aligned}
$$

Thus, the first row of the table $C$ has at most $k$ non-zero. Next we prove that each one of the remaining rows has at most $k+2$ non-zero terms. For every $i=1, \ldots, m-1$ we compute

$$
\gamma \cdot \gamma^{q^{i}}=\left(\frac{1}{n+1} \beta-\frac{k}{n+1}\right) \cdot\left(\frac{1}{n+1} \beta^{q^{i}}-\frac{k}{n+1}\right)
$$




$$
\begin{aligned}
= & \frac{1}{(n+1)^{2}} \beta \cdot \beta^{q^{i}}-\frac{k}{(n+1)^{2}} \beta-\frac{k}{(n+1)^{2}} \beta^{q^{i}}+\frac{k^{2}}{(n+1)^{2}} \\
= & \frac{1}{(n+1)^{2}}\left(\beta^{q^{\lambda_{0}}}+\beta^{q^{\lambda_{1}}}+\cdots+\beta^{q^{\lambda_{k-1}}}\right)-\frac{k}{(n+1)^{2}} \beta-\frac{k}{(n+1)^{2}} \beta^{q^{i}} \\
& +\frac{k^{2}}{(n+1)^{2}} \\
= & \frac{1}{(n+1)}\left(\left(\frac{1}{n+1} \beta^{q^{\lambda_{0}}}-\frac{k}{n+1}\right)+\cdots+\left(\frac{1}{n+1} \beta^{q^{\lambda_{k-1}}}-\frac{k}{n+1}\right)\right)+ \\
& +\frac{2 k^{2}}{(n+1)^{2}}-\frac{k}{(n+1)^{2}} \beta-\frac{k}{(n+1)^{2}} \beta^{q^{i}} \\
= & \frac{1}{(n+1)}\left(\gamma^{q^{\lambda_{0}}}+\gamma^{q^{\lambda_{1}}}+\cdots+\gamma^{q^{\lambda_{k-1}}}\right)-\frac{k}{n+1} \gamma-\frac{k}{n+1} \gamma^{q^{i}} .
\end{aligned}
$$

Hence, the multiplication table has at most $(k+2) \cdot(m-1)+k=(k+2) m-2$ non-zero terms, so the complexity is at most $(k+2) m-2$.

Finally, for the row $(m-i)$ of the table $C$, using Theorem 4.2.1 we get,

$$
\begin{aligned}
\gamma \cdot \gamma^{q^{m-i}}= & \left(\frac{1}{n+1} \beta-\frac{k}{n+1}\right) \cdot\left(\frac{1}{n+1} \beta^{q^{m-i}}-\frac{k}{n+1}\right) \\
= & \frac{1}{(n+1)^{2}} \beta \cdot \beta^{q^{m-i}}-\frac{k}{(n+1)^{2}} \beta-\frac{k}{(n+1)^{2}} \beta^{q^{m-i}}+\frac{k^{2}}{(n+1)^{2}} \\
= & \frac{1}{(n+1)^{2}}\left(\beta^{q^{\lambda_{k-1-i}}}+\cdots+\beta^{q^{\lambda_{0-i}}}\right)-\frac{k}{(n+1)^{2}} \beta-\frac{k}{(n+1)^{2}} \beta^{q^{m-i}} \\
& +\frac{k^{2}}{(n+1)^{2}} \\
= & \frac{1}{(n+1)}\left(\left(\frac{1}{n+1} \beta^{q^{\lambda_{k-1-i}}}-\frac{k}{n+1}\right)+\cdots+\left(\frac{1}{n+1} \beta^{q^{\lambda_{0-i}}}-\frac{k}{n+1}\right)\right) \\
& +\frac{2 k^{2}}{(n+1)^{2}}-\frac{k}{(n+1)^{2}} \beta-\frac{k}{(n+1)^{2}} \beta^{q^{m-i}} \\
= & \frac{1}{(n+1)}\left(\gamma^{q^{\lambda_{k-1-i}}}+\cdots+\gamma^{q^{\lambda_{0}-i}}\right)-\frac{k}{n+1} \gamma-\frac{k}{n+1} \gamma^{q^{-i}} .
\end{aligned}
$$

Thus, the row $j$ of the multiplication table of $\gamma$ is a cyclic permutation of $j$ positions of row $(m-j)$. 
We note that the above proof is analogous in the case where $q$ is even with the exception that the first row contributes only 1 to the complexity as $\gamma \cdot \gamma=\gamma^{2}$ is an element of the normal basis generated by $\gamma$. Also, when $m$ is even, we must use the bound that the $m / 2$ row of the multiplication table $C$ has at most $m$ non-zero entries. The resulting complexity for $q$ odd is bounded above by $(m-2) \cdot(k+2)+m+k=$ $m(k+3)-k-4$, and for $q$ even is bounded above by $(k+3) m-2 k-3$.

Recall that the coefficients $t_{i}$ of the polynomial $h(x)$ defined in Theorem 4.3.1 are given by

$$
t_{i}=\operatorname{Tr}_{q^{m} / q}\left(\beta \cdot \beta^{q^{i}}\right), i=0,1, \ldots, m-1
$$

If $\beta$ generates a Type II optimal normal bases, by Theorem 4.2 .3 there exist $\lambda_{i}, \mu_{i} \in \mathbb{Z}_{n}$ such that

$$
\beta \cdot \beta^{2^{j}}=\beta^{2^{\lambda_{0}}}+\beta^{2^{\mu_{0}}}+\cdots+\beta^{\lambda_{k-1}}+\beta^{\mu_{k-1}} .
$$

Thus, $t_{0}=1$ and $t_{i}=0$ for $1 \leq i \leq m-1$. This provides the analogous result for Type II optimal normal bases.

Theorem 4.3.2. Let $\alpha \in \mathbb{F}_{q^{n}}$ generate an optimal normal basis of Type II of $\mathbb{F}_{q^{n}}$ over $\mathbb{F}_{q}$ and let $\beta=\operatorname{Tr}_{q^{n} / q^{m}}(\alpha) \in \mathbb{F}_{q^{m}}$ with $m=n / k, k \leq m$. Then $\beta$ is self-dual and consequently the complexity of the dual basis of $\beta$ is $2 k(m-1)+1$.

We summarize the results in this section in the following table. 


\begin{tabular}{|l|l|l|l|}
\hline & Type I $(q$ odd $)$ & Type I $(q$ even $)$ & Type II $(q$ even $)$ \\
\hline$m$ odd & $(k+2) m-2$ & $(k+2)(m-1)+1$ & $2 k(m-1)+1$ \\
$m$ even & $(k+3) m-k-4$ & $(k+3) m-2 k-3$ & $2 k(m-1)+1$ \\
\hline
\end{tabular}

Table 4.1: Upper bounds on complexities for $\mathbb{F}_{q^{m}}$ obtained by the dual of the trace of ONBs, where $m=n / k$.

\subsection{Existence of Optimal Extensions}

A question that naturally arises is whether, given a prime power $q$ and a natural number $m$, there exists an extension $\mathbb{F}_{q^{n}}$ of $\mathbb{F}_{q^{m}}$, such that $\mathbb{F}_{q^{n}}$ has an optimal normal basis over $\mathbb{F}_{q}$. This is a hard question, and certainly it is not the subject of this work. We give a brief discussion of known results that provide partial answers to this and related questions.

Dirichlet's prime number theorem states that for any two integers $a, d$ which are relatively prime, there exist infinitely many primes congruent to $a(\bmod d)$. Under the Generalized Riemann Hypothesis, for every prime $p$ there is a primitive root $(\bmod p)$ which is less than $70(\ln p)^{2}$.

The extension $\mathbb{F}_{q^{n}}$ contains a Type I optimal normal basis over $\mathbb{F}_{q}$ implies that $n=\ell-1$ for a prime $\ell$ and $q$ is primitive modulo $\ell$. The requirement that $\mathbb{F}_{q^{n}}$ is an extension of $\mathbb{F}_{q^{m}}$ implies that $\ell \equiv 1(\bmod m)$. One would be interested to know if such a prime always exists and what is its order of magnitude in terms of $m$. This is already a refinement of Artin's conjecture on primitive roots which states that an integer $b \neq 1,-1$ which is not a perfect square is a primitive root modulo infinitely many primes [32]. We note further, that if $q$ is a square it cannot be primitive modulo any odd prime. Suppose that $q$ is an odd prime power. Then the work of Moree [30] 
and Lenstra [24], imply that under the Generalized Riemann Hypothesis (GRH) there exist infinitely many primes $\ell$ such that $\ell \equiv 1(\bmod m)$ and $q$ is primitive modulo $\ell$. Thus, under the GRH, one is assured that Type I Optimal extensions such as those used in this work exist.

The extension $\mathbb{F}_{2^{n}}$ contains a Type II optimal normal basis of $\mathbb{F}_{2}$ implies that $n=1 / 2(\ell-1)$ for a prime $\ell$ and that either 2 is primitive modulo $\ell$ or $n \equiv 1(\bmod 4)$ (equivalently $\ell \equiv 3(\bmod 4))$ and that 2 has multiplicative order $n$ modulo $\ell$. In the first case we can apply the same argument as in the Type I case above. In the second case the works of Lenstra and Moree do not apply: we still require that $\ell \equiv 1$ $(\bmod m)$, but now we require that 2 generates the quadratic residues modulo $\ell$.

The ratio $k=n / m$ is clearly of importance for the bounds that we have given. In the terminology of this section one would like to know the smallest prime $\ell$ in the arithmetic progression of 1 modulo $m$ such that $q$ is primitive modulo $\ell$. This however is a much harder question, and a good bound seems to be out of reach even under the GRH.

\subsection{Summary and Comparisons}

In this chapter we give low complexity normal elements for $\mathbb{F}_{q^{m}}$ over $\mathbb{F}_{q}$, when $m=n / k$ and there is an optimal normal element in $\mathbb{F}_{q^{n}}$. Table 4.2 gives a summary of the complexities obtained in this paper.

In practice, we are mainly interested in fields with $q$ even where we have low 


\begin{tabular}{|l|l|l|l|}
\hline & Type I $(q$ odd $):$ & Type I $(q$ even $):$ & Type II $(q$ even $):$ \\
\hline$m$ odd & $(k+1) m-k$ & $k m-k+1$ & $2 k m-2 k+1$ \\
$m$ even, $k$ odd & $(k+2) m-3 k+1$ & $(k+1) m-3 k+2$ & (for all $m)$ \\
$m$ even, $k$ even & $(k+1) m-k$ & $k m-k+1$ & \\
\hline
\end{tabular}

Table 4.2: Summary of best-case low complexities for $\mathbb{F}_{q^{m}}$ obtained by traces, where $m=n / k$.

complexity normal bases. As a result of our constructions, we are able to find low complexity normal elements in intermediate fields using tables from [10]. The optimal normal bases in characteristic two were completely characterized in [12], but there is still a need to find low complexity normal bases in extensions for which there is no optimal normal basis. Appendix D gives $n$-degree extensions of $\mathbb{F}_{2}, 278 \leq n \leq 1026$, in which there exists a Type II optimal normal basis in $\mathbb{F}_{2^{n}}$ but no such basis exists in $\mathbb{F}_{2^{n / 2}}$. We also provide the resulting complexities of the found bases.

The National Institute of Standards and Technology (NIST) recommends a series of five elliptic curves over binary fields for United States federal government use in cryptography [33]. The complexities of the normal basis representatives were found by [26], and Table 4.3 compares our best-found constructions with the NIST standard curves.

\begin{tabular}{|l|l|l|}
\hline$m$ & NIST $-c_{N}$ & Our $c_{N}$ \\
\hline 163 & 645 & 649 \\
233 & $465^{*}$ & $465^{*}$ \\
283 & 1677 & - \\
307 & - & 1225 \\
409 & 1629 & 1633 \\
571 & 5637 & - \\
577 & - & 2305 \\
\hline
\end{tabular}

Table 4.3: Comparison of NIST-standard normal basis representatives of $\mathbb{F}_{2^{m}}$ over $\mathbb{F}_{2}$ with our construction. 
The complexities of the extensions where $m=163$ and $m=409$ given by NIST satisfy the relation $c_{N}=4 m-7$, which is due to the construction in Theorem 2.5.3. This construction requires finding primitive roots of unity in large composite extensions of $\mathbb{F}_{q^{n}}$, which is certainly computationally more difficult than finding the trace of a known optimal normal element. The basis used for $m=233$ is a Type II ONB, and for the $m=283$ and $m=571$ existing tables in [10] only give extensions for which ONBs exist up to $m=2000$, and so we could not apply our construction. We have provided complexities using our construction for extensions $m=307$ and $m=577$ which have the properties that their degrees are prime, close to an extension given by NIST, and the order of the multiplicative group $2^{m}-1$ has no small prime factors. This could be indicative that elliptic curve cryptography is computationally desirable over these and similar fields. However, further research into the cryptographic strength of these fields is necessary. 


\section{Chapter 5}

\section{Conclusions}

\subsection{Summary of Results}

We examine the distribution of normal bases in finite fields of characteristic two by running an exhaustive search for normal elements for all extension degrees $n \leq 39$. The search is significantly improved by the use of a Gray code ordering. We perform exhaustive searches of all self-dual normal bases for $n \leq 37$. We count the number of normal bases, tabulate the complexities of all bases and calculate their average and variance. We give plots of the number of normal bases versus the complexity of a normal basis. We use the above data to form the following conjectures.

- The number of normal bases of $\mathbb{F}_{2^{n}}$ over $\mathbb{F}_{2}$ are normally distributed with respect to their complexities.

- The average complexity of a normal basis of $\mathbb{F}_{2^{n}}$ over $\mathbb{F}_{2}, n \geq 8$ is bounded 
above by $\left(n^{2}-n+3\right) / 2$.

- The variance of the complexities of normal bases of $\mathbb{F}_{2^{n}}$ over $\mathbb{F}_{2}$ has a strong positive correlation with the average complexity of a normal basis. Loosely, the variance of the complexities of normal bases of $\mathbb{F}_{2^{n}}$ over $\mathbb{F}_{2}$ is bounded above by $n^{2} / 2$ for sufficiently large $n$.

- There is no constant $k$ such that the minimum complexity of a normal basis of $\mathbb{F}_{2^{n}}$ over $\mathbb{F}_{2}$ is bounded below by $k \cdot n$ for all $n$.

We give new low complexity normal bases in subfields $\mathbb{F}_{q^{m}}$ of $\mathbb{F}_{q^{n}}$, where $n=m k$ for some $k$ and $\mathbb{F}_{q^{n}}$ contains an Optimal normal basis. This projection is performed by means of the field trace function. We do this for both even and odd characteristics and for both Type I and Type II Optimal normal bases. We also give the complexity of the dual bases of all bases constructed. Fixing $n$, these complexities depend on $k$ and are summarized in the following tables.

\begin{tabular}{|l|l|l|l|}
\hline & Type I $(q$ odd $):$ & Type I $(q$ even $):$ & Type II $(q$ even $):$ \\
\hline$m$ odd & $(k+1) m-k$ & $k m-k+1$ & $2 k m-2 k+1$ \\
$m$ even, $k$ odd & $(k+2) m-3 k+1$ & $(k+1) m-3 k+2$ & (for all $m)$ \\
$m$ even, $k$ even & $(k+1) m-k$ & $k m-k+1$ & \\
\hline
\end{tabular}

Table 5.1: Summary of best-case low complexities for $\mathbb{F}_{q^{m}}$ obtained by traces, where $m=n / k$.

\begin{tabular}{|l|l|l|l|}
\hline & Type I $(q$ odd $)$ & Type I $(q$ even $)$ & Type II $(q$ even $)$ \\
\hline$m$ odd & $(k+2) m-2$ & $(k+2)(m-1)+1$ & $2 k(m-1)+1$ \\
$m$ even & $(k+3) m-k-4$ & $(k+3) m-2 k-3$ & $2 k(m-1)+1$ \\
\hline
\end{tabular}

Table 5.2: Upper bounds on complexities for $\mathbb{F}_{q^{m}}$ obtained by the dual of the trace of ONBs, where $m=n / k$. 


\subsubsection{Explanation of Tables in Appendices B and C}

Appendix B shows findings on the complexity of normal elements for every finite field $\mathbb{F}_{2^{n}}$ with $n \leq 39$, computed with the implementation of the GrayCodeNCD algorithm. Due to the field size and the computational complexity, no search was performed for self-dual normal elements for $n \geq 37$. The optimal normal bases found are in agreement with the theorem of Gao and Lenstra [12] that characterizes for which fields optimal normal bases exist.

Appendix $\mathrm{C}$ is devoted to the cases when $40 \leq n \leq 607$. For these values of $n$, no self-dual testing has been performed. When an optimal normal element is known not to exist, we show the lowest complexity found by using three methods. In the first one we apply Theorem 2.5.1 which allows us to combine two complexities previously computed. Secondly, we apply the results obtained in Chapter 4. The final method is based on a random search, which starts with a random finite field element, and randomly flips one of its polynomial coefficients a prescribed number of times, keeping the normal element found with lowest complexity. If the first method yields the lowest complexity then we indicate in the Property column which co-prime factorization of $n$ led us to obtain the low complexity. If the second method yields the smaller complexity we note this in the Property column by "TraceONB", and indicate both the type of the optimal normal basis and the value of $k$ required. Theorem 2.5.1 does not apply for $n$ prime or prime-power, and or these values the random search is required. However, random search yields quite large minimum complexities, so it 
appears only when the other methods fail. We observe that the smallest complexity found is not necessarily the minimum complexity possible in the field, and for this reason we denote best found complexity as $\min c_{N}$.

\subsection{Further Work and Questions}

This discussion of normal bases, their distribution and some constructions of lowcomplexity of normal bases lends itself to even more questions on these topics.

Question 1. Can the conjectures in Chapter 3 be refined or proven? In particular, can it be shown that normal bases of $\mathbb{F}_{2^{n}}$ over $\mathbb{F}_{2}$ are normally distributed with respect to their complexities? If so, can we envelope this bound and find a probabilistic (at worst) or deterministic (at best) upper-bound on the lowest-expected complexity of $\mathbb{F}_{2^{n}}$ over $\mathbb{F}_{2}$ ?

Question 2. There is an increasing interest in $\mathbb{F}_{3}$ for use in pairing-based cryptosystems (see $[2,7])$, and in the use of elliptic curves over prime fields (see [37]). The natural extension is to ask if the above conjectures hold for general $q$. It should be noted that experiments over higher $q$, even $q=3$ and moderate $n$, are computationally out of reach using the methods presented here.

Question 3. It was noted in the cases $n=37,38,39$, when the Gray code ordering was split across multiple machines, that there were large blocks of the field (under the Gray code ordering) where no normal elements were obtained. This indicates 
some regular structure on normal elements. Similar to work performed by Cohen [5] on finding primitive normal polynomials with prescribed coefficients, it appears that normal elements could be found with prescribed structure. This seems very difficult, however, even in the binary case.

Question 4. What can be said about the orders of certain normal elements? It seems, from tables obtained in the research pertaining to Chapter 3 , that often lowcomplexity normal elements are primitive, but when is this true, in general? The primitive normal element theorem states that in all finite fields there exists a normal element that generates the multiplicative group of the field. Is it desirable to find these elements? Cohen [5] attempts to answer this question, though from an existence point of view.

The conjectures given in Chapter 3 and the random search in Appendix C indicate that, though normal elements are relatively dense in finite fields, specific constructions are required to be able to find normal elements of low-complexity.

Question 5. It is known that all linear transformations from $\mathbb{F}_{q^{n}}$ into $\mathbb{F}_{q}$ are precisely the maps $L_{\beta}, \beta \in \mathbb{F}_{q^{n}}$ where $L_{\beta}(\alpha)=\operatorname{Tr}_{q^{n} / q}(\beta \cdot \alpha)$ for all $\alpha \in \mathbb{F}_{q^{n}}$. Using the methods of Chapter 4, are there any such $\beta \in \mathbb{F}_{q^{n}}$ such that the projection $L_{\beta}(\alpha)$ gives a low-complexity normal basis?

Question 6. In a similar vein, are there other projection maps $\mathbb{F}_{q^{n}}$ to $\mathbb{F}_{q^{m}}$ that give similar results? The natural next candidate is the field norm function (the multiplicative version of the trace). It is simple to see due to the multiplicative nature of 
the norm and the cyclic structure imposed by the Frobenius q-automorphism that the conjugates of the norm of a normal element fail to be linearly independent. Other more complex projection maps may still exist.

Question 7. This is restating Section 4.4. The question is, given any desired $q$ and extension degree $m$, what is the smallest $k$ such that $\mathbb{F}_{q^{n}}=\mathbb{F}_{q^{m k}}$ admits an optimal normal basis? Does such a $k$ exist for all $q$ and $m$ ? This is a deep question related to the Generalized Riemann Hypothesis, but a study for certain desired values of $q$ and $m$ could be of interest.

Question 8. In Section 4.5 we give a table of field extensions recommended by NIST for elliptic curve cryptography and compare them to fields which have some similar properties (such as prime degree, etc.) but to which the traces obtained in Chapter 4 yield low complexity normal bases. A further study of these fields is necessary to determine their cryptographic strength. If not, are there other fields with similar properties to which our construction applies? 


\section{Appendix A}

\section{Optimal Normal Bases over $\mathbb{F}_{2}$}

\begin{tabular}{|l|l|l|l|l|l|l|l|l|l|}
\hline $2 \dagger$ & 113 & 293 & 473 & $676 *$ & 873 & 1110 & 1310 & 1533 & 1790 \\
3 & 119 & 299 & 483 & 683 & $876 *$ & $1116 *$ & 1323 & 1539 & 1791 \\
$4 *$ & $130 *$ & 303 & $490 *$ & 686 & 879 & 1118 & 1329 & 1541 & 1806 \\
5 & 131 & 306 & 491 & 690 & $882 *$ & 1119 & 1331 & $1548 *$ & 1811 \\
6 & 134 & 309 & 495 & $700 *$ & 891 & 1121 & 1338 & 1559 & 1818 \\
9 & 135 & $316 *$ & $508 *$ & $708 *$ & 893 & $1122 *$ & 1341 & $1570 *$ & 1821 \\
$10 *$ & $138 *$ & 323 & 509 & 713 & $906 *$ & 1133 & 1346 & 1583 & 1829 \\
11 & 146 & 326 & 515 & 719 & 911 & 1134 & 1349 & 1593 & 1835 \\
$12 *$ & $148 *$ & 329 & 519 & 723 & 923 & 1146 & 1353 & 1601 & 1838 \\
14 & 155 & 330 & $522 *$ & 725 & 930 & 1154 & 1355 & $1618 *$ & 1845 \\
$18 \dagger$ & 158 & 338 & 530 & 726 & 933 & 1155 & 1359 & $1620 *$ & 1850 \\
23 & $162 *$ & $346 *$ & 531 & 741 & 935 & 1166 & 1370 & 1626 & 1854 \\
26 & $172 *$ & $348 *$ & $540 *$ & 743 & 938 & 1169 & $1372 *$ & $1636 *$ & 1859 \\
$28 *$ & 173 & 350 & 543 & 746 & 939 & $1170 *$ & $1380 *$ & 1649 & $1860 *$ \\
29 & 174 & 354 & 545 & 749 & $940 *$ & 1178 & 1394 & 1653 & 1863 \\
30 & $178 *$ & 359 & $546 *$ & 755 & $946 *$ & 1185 & 1398 & 1659 & $1866 \dagger$ \\
33 & 179 & 371 & 554 & $756 *$ & 950 & $1186 *$ & 1401 & 1661 & $1876 *$ \\
35 & $180 *$ & $372 *$ & $556 *$ & 761 & 953 & 1194 & 1409 & $1666 *$ & 1883 \\
$36 *$ & 183 & 375 & 558 & 765 & 965 & 1199 & 1418 & $1668 *$ & 1889 \\
39 & 186 & $378 \dagger$ & 561 & 771 & 974 & 1211 & 1421 & 1673 & 1898 \\
41 & 189 & $378 *$ & $562 *$ & $772 *$ & 975 & $1212 *$ & 1425 & 1679 & $1900 *$ \\
50 & 191 & 386 & 575 & 774 & 986 & 1218 & $1426 *$ & 1685 & 1901 \\
51 & 194 & $388 *$ & 585 & 779 & 989 & 1223 & 1430 & $1692 *$ & $1906 *$ \\
$52 *$ & $196 *$ & 393 & $586 *$ & 783 & 993 & $1228 *$ & 1439 & 1703 & 1923 \\
53 & 209 & 398 & 593 & 785 & 998 & 1229 & 1443 & 1706 & 1925 \\
$58 *$ & $210 \dagger$ & 410 & 606 & $786 *$ & 1013 & 1233 & $1450 *$ & 1730 & 1926 \\
$60 *$ & 221 & 411 & 611 & 791 & 1014 & $1236 *$ & 1451 & $1732 *$ & $1930 *$ \\
65 & $226 *$ & 413 & $612 *$ & $796 *$ & $1018 *$ & 1238 & $1452 *$ & 1733 & 1931 \\
$66 *$ & 230 & 414 & 614 & 803 & 1019 & 1251 & 1454 & 1734 & 1938 \\
69 & 231 & $418 *$ & 615 & 809 & 1026 & $1258 *$ & 1463 & $1740 *$ & $1948 *$ \\
74 & 233 & 419 & $618 \dagger$ & 810 & 1031 & 1265 & 1469 & 1745 & 1953
\end{tabular}




\begin{tabular}{|l|l|l|l|l|l|l|l|l|l|}
81 & 239 & $420 *$ & 629 & 818 & 1034 & 1269 & 1478 & $1746 *$ & 1955 \\
$82 *$ & 243 & 426 & 638 & $820 *$ & 1041 & 1271 & 1481 & 1749 & 1958 \\
83 & 245 & 429 & 639 & $826 *$ & 1043 & 1274 & $1482 *$ & 1755 & 1959 \\
86 & 251 & 431 & 641 & $828 *$ & 1049 & 1275 & $1492 *$ & 1758 & 1961 \\
89 & 254 & 438 & 645 & 831 & 1055 & $1276 *$ & $1498 *$ & 1763 & 1965 \\
90 & 261 & 441 & 650 & 833 & $1060 *$ & 1278 & 1499 & 1766 & $1972 *$ \\
95 & $268 *$ & $442 *$ & 651 & 834 & 1065 & $1282 *$ & 1505 & 1769 & 1973 \\
98 & 270 & 443 & $652 *$ & 846 & 1070 & 1289 & 1509 & 1773 & $1978 *$ \\
99 & 273 & 453 & 653 & $852 *$ & $1090 *$ & $1290 *$ & 1511 & 1778 & 1983 \\
$100 *$ & 278 & $460 *$ & $658 *$ & $858 *$ & 1103 & 1295 & 1518 & 1779 & $1986 *$ \\
105 & 281 & $466 *$ & 659 & 866 & 1106 & $1300 *$ & $1522 *$ & 1785 & 1994 \\
$106 *$ & $292 *$ & 470 & $660 *$ & 870 & $1108 *$ & $1306 *$ & $1530 *$ & $1786 *$ & $1996 *$ \\
\hline
\end{tabular}

* Denotes existence of a Type I optimal normal basis

$\dagger \quad$ Denotes existence of both Type I and Type II optimal normal bases 


\section{Appendix B}

\section{Exhaustive Search of $\mathbb{F}_{2^{n}, n<40}$}

This table displays the results found by the exhaustive search described in Chapter 3. The table gives the number of normal bases found $\left(\left(\Phi_{2}\left(x^{n}-1\right)\right) / n\right)$, smallest and largest complexities $\left(m_{c_{N}}, M_{c_{N}}\right)$, average complexity, variance and standard deviation $\left(\operatorname{Avg}_{c_{N}}, \operatorname{Var}_{c_{N}}, \sigma_{c_{N}}\right)$ of all complexities, then the smallest and largest complexities for self-dual normal elements.

\begin{tabular}{|c|c|c|c|c|c|c|c|c|c|}
\hline \multirow[b]{2}{*}{$\mathbf{n}$} & \multirow[b]{2}{*}{$\frac{\Phi_{2}\left(x^{n}-1\right)}{n}$} & \multirow[b]{2}{*}{$\boldsymbol{m}_{c_{N}}$} & \multirow[b]{2}{*}{$M_{C_{N}}$} & \multirow[b]{2}{*}{$A v g_{c_{N}}$} & \multirow[b]{2}{*}{$\operatorname{Var}_{c_{N}}$} & \multirow[b]{2}{*}{$\sigma_{c_{N}}$} & \multicolumn{2}{|c|}{ self-dual } & \multirow[b]{2}{*}{ Notes } \\
\hline & & & & & & & $m_{c_{N}}$ & $M_{c_{N}}$ & \\
\hline 2 & 1 & 3 & 3 & 3.00 & 0 & 0 & 3 & 3 & Optimal, sd \\
\hline 3 & 1 & 5 & 5 & 5.00 & 0 & 0 & 5 & 5 & Optimal, sd \\
\hline 4 & 2 & 7 & 9 & 8.00 & 1.00 & 1.00 & - & - & \\
\hline 5 & 3 & 9 & 15 & 11.67 & 6.20 & 2.49 & 9 & 9 & Optimal, sd \\
\hline 6 & 4 & 11 & 17 & 15.00 & 6.00 & 2.45 & 11 & 15 & Optimal, sd \\
\hline 7 & 7 & 19 & 27 & 23.00 & 9.12 & 3.02 & 21 & 21 & $3 n-2$ \\
\hline 8 & 16 & 21 & 35 & 29.00 & 11.02 & 3.32 & - & - & $3 n-3$ \\
\hline 9 & 21 & 17 & 45 & 35.57 & 41.6 & 6.45 & 17 & 29 & Optimal, sd \\
\hline 10 & 48 & 19 & 61 & 44.83 & 61.31 & 7.83 & 27 & 51 & \\
\hline 11 & 93 & 21 & 71 & 55.82 & 57.61 & 7.59 & 21 & 57 & Optimal, sd \\
\hline 12 & 128 & 23 & 83 & 64.13 & 139.48 & 11.81 & - & - & \\
\hline 13 & 315 & 45 & 101 & 78.38 & 71.06 & 8.43 & 45 & 81 & Best, sd \\
\hline 14 & 448 & 27 & 135 & 91.07 & 108.37 & 10.41 & 27 & 135 & Optimal, sd \\
\hline 15 & 675 & 45 & 137 & 105.89 & 127.46 & 11.29 & 45 & 105 & Best, sd \\
\hline 16 & 2048 & 85 & 157 & 115.82 & 731.7 & 27.05 & - & - & \\
\hline 17 & 3825 & 81 & 177 & 132.77 & 671.84 & 25.92 & 81 & 171 & Best, sd \\
\hline 18 & 5376 & 35 & 243 & 153.51 & 189.5 & 13.77 & 35 & 243 & Optimal, sd \\
\hline 19 & 13797 & 117 & 229 & 172.00 & 174.05 & 13.19 & 117 & 201 & Best, sd \\
\hline 20 & 24576 & 63 & 257 & 190.80 & 207.28 & 14.40 & - & - & \\
\hline 21 & 27783 & 95 & 277 & 210.97 & 216.43 & 14.71 & 105 & 237 & \\
\hline 22 & 95232 & 63 & 363 & 231.93 & 238.56 & 15.45 & 63 & 363 & $3 n-3$ \\
\hline 23 & 182183 & 45 & 325 & 254.02 & 254.6 & 15.96 & 45 & 309 & Optimal, sd \\
\hline 24 & 262144 & 105 & 375 & 276.82 & 281.01 & 16.76 & - & - & \\
\hline 25 & 629145 & 93 & 383 & 301.01 & 300.37 & 17.33 & 93 & 357 & Best, sd \\
\hline 26 & 1290240 & 51 & 555 & 325.96 & 328.59 & 18.13 & 51 & 555 & Optimal, sd \\
\hline 27 & 1835001 & 141 & 443 & 351.99 & 351.38 & 18.75 & 141 & 413 & \\
\hline 28 & 3670016 & 55 & 517 & 378.98 & 379.12 & 19.47 & - & - & Optimal \\
\hline 29 & 9256395 & 57 & 521 & 407.00 & 406.22 & 20.15 & 57 & 465 & Optimal, sd \\
\hline 30 & 11059200 & 59 & 759 & 435.95 & 438.52 & 20.94 & 59 & 759 & Optimal, sd \\
\hline 31 & 28629151 & 237 & 587 & 466.00 & 465.2 & 21.57 & 237 & 537 & Best, sd \\
\hline 32 & 67108864 & 361 & 621 & 497.00 & 495.95 & 22.27 & - & - & \\
\hline 33 & 97327197 & 65 & 693 & 529.00 & 528.48 & 22.99 & 65 & 693 & Optimal, sd \\
\hline
\end{tabular}




\begin{tabular}{|c|c|c|c|c|c|c|c|c|c|}
\hline & & & & & & & \multicolumn{2}{|c|}{ self-dual } & \multirow[b]{2}{*}{ Notes } \\
\hline $\mathrm{n}$ & $\frac{\Phi_{2}\left(x^{n}-1\right)}{n}$ & $m_{c_{N}}$ & $M_{c_{N}}$ & $A v g_{\mathrm{c}_{N}}$ & $\operatorname{Var}_{c_{N}}$ & $\sigma_{c_{N}}$ & $m_{c_{N}}$ & $M_{c_{N}}$ & \\
\hline 34 & 250675200 & 243 & 819 & 562.00 & 561.52 & 23.70 & 243 & 819 & Best, sd \\
\hline 35 & 352149515 & 69 & 779 & 596.00 & 595.03 & 24.39 & 69 & 693 & Optimal, sd \\
\hline 36 & 704643060 & 71 & 1017 & 630.99 & 630.51 & 25.11 & - & - & Optimal \\
\hline 37 & 1857283155 & 171 & 823 & 667.00 & 666.04 & 25.81 & & & \\
\hline 38 & 3616800703 & 207 & 1131 & 704.00 & 703.18 & 26.52 & & & \\
\hline 39 & 5282242828 & 77 & 933 & 742.00 & 741.09 & 27.22 & & & Optimal \\
\hline
\end{tabular}

101

Reproduced with permission of the copyright owner. Further reproduction prohibited without permission. 


\section{Appendix C}

\section{Complexities Found $40 \leq n \leq 607$}

\begin{tabular}{|c|c|c|c|c|c|c|c|}
\hline$n$ & $\min c_{N}$ & Property & Method & $n$ & $\min c_{N}$ & Property & Method \\
\hline 40 & 189 & 5,8 & Thm 2.5.1 & 84 & 275 & 3,28 & Thm 2.5.1 \\
\hline 41 & 81 & Optimal & {$[31]$} & 85 & 729 & 5,17 & Thm 2.5.1 \\
\hline 42 & 135 & 3,14 & Thm 2.5.1 & 86 & 171 & Optimal & {$[31]$} \\
\hline 43 & 169 & TraceONB & Type $2, \mathrm{k}=2$ & 87 & 285 & 3,29 & Thm 2.5.1 \\
\hline 44 & 147 & 4,11 & Thm 2.5.1 & 88 & 441 & 8,11 & Thm 2.5.1 \\
\hline 45 & 153 & 5,9 & Thm 2.5.1 & 89 & 177 & Optimal & {$[31]$} \\
\hline 46 & 135 & 2,23 & Thm 2.5.1 & 90 & 179 & Optimal & [31] \\
\hline 47 & 645 & TraceONB & Type 2, $\mathrm{k}=7$ & 91 & 541 & TraceONB & Type $2, \mathrm{k}=3$ \\
\hline 48 & 425 & 3,16 & Thm 2.5.1 & 92 & 315 & 4,23 & Thm 2.5.1 \\
\hline 49 & 193 & TraceONB & Type $2, \mathrm{k}=2$ & 93 & 369 & TraceONB & Type $2, \mathrm{k}=2$ \\
\hline 50 & 99 & Optimal & {$[31]$} & 94 & 931 & TraceONB & Type $2, \mathrm{k}=5$ \\
\hline 51 & 101 & Optimal & [31] & 95 & 189 & Optimal & [31] \\
\hline 52 & 103 & Optimal & {$[31]$} & 96 & 1805 & 3,32 & Thm 2.5.1 \\
\hline 53 & 105 & Optimal & {$[31]$} & 97 & 385 & TraceONB & Type $2, \mathrm{k}=2$ \\
\hline 54 & 209 & TraceONB & Type $1, \mathrm{k}=3$ & 98 & 195 & Optimal & {$[31]$} \\
\hline 55 & 189 & 5,11 & Thm 2.5.1 & 99 & 197 & Optimal & {$[31]$} \\
\hline 56 & 399 & 7,8 & Thm 2.5.1 & 100 & 199 & Optimal & {$[31\}$} \\
\hline 57 & 585 & 3,19 & Thm 2.5.1 & 101 & 601 & TraceONB & Tyре $2, \mathrm{k}=3$ \\
\hline 58 & 115 & Optimal & {$[31]$} & 102 & 303 & 2,51 & Thm 2.5.1 \\
\hline 59 & 697 & TraceONB & Type $2, k=6$ & 103 & 613 & TraceONB & Type $2, \mathrm{k}=3$ \\
\hline 60 & 119 & Optimal & {$[31]$} & 104 & 945 & 8,13 & Thm 2.5.1 \\
\hline 61 & 361 & TraceONB & Type $2, \mathrm{k}=3$ & 105 & 209 & Optimal & {$[31]$} \\
\hline 62 & 367 & TraceONB & Type $2, \mathrm{k}=3$ & 106 & 211 & Optimal & {$[31]$} \\
\hline 63 & 323 & 7,9 & Thm 2.5.1 & 107 & 1485 & TraceONB & Type $2, \mathrm{k}=7$ \\
\hline 64 & 1829 & Prime Power & Random & 108 & 635 & TraceONB & Type $1, \mathrm{k}=5$ \\
\hline 65 & 129 & Optimal & {$[31]$} & 109 & 1081 & TraceONB & Type $2, \mathrm{k}=5$ \\
\hline 66 & 131 & Optimal & {$[31]$} & 110 & 399 & 10,11 & Thm 2.5.1 \\
\hline 67 & 265 & TraceONB & Type $2, \mathrm{k}=2$ & 111 & 2201 & TraceONB & Type $2, k=10$ \\
\hline 68 & 567 & 4,17 & Thm 2.5.1 & 112 & 1615 & 7,16 & Thm 2.5.1 \\
\hline 69 & 137 & Optimal & {$[31]$} & 113 & 225 & Optimal & [31] \\
\hline 70 & 207 & 2,35 & Thm 2.5.1 & 114 & 1287 & 6,19 & Thm 2.5.1 \\
\hline 71 & 841 & TraceONB & Type $2, \mathrm{k}=6$ & 115 & 405 & 5,23 & Thm 2.5.1 \\
\hline 72 & 357 & 8,9 & Thm 2.5.1 & 116 & 399 & 4,29 & Thm 2.5.1 \\
\hline 73 & 289 & TraceONB & Type $2, \mathrm{k}=2$ & 117 & 765 & 9,13 & Thm 2.5.1 \\
\hline 74 & 147 & Optimal & {$[31]$} & 118 & 703 & TraceONB & Type $2, \mathrm{k}=3$ \\
\hline 75 & 465 & 3,25 & Thm 2.5.1 & 119 & 237 & Optimal & {$[31]$} \\
\hline 76 & 819 & 4,19 & Thm 2.5.1 & 120 & 945 & 3,40 & Thm 2.5.1 \\
\hline 77 & 399 & 7,11 & Thm 2.5.1 & 121 & 1441 & TraceONB & Type $2, \mathbf{k}=6$ \\
\hline 78 & 231 & 2,39 & Thm 2.5.1 & 122 & 4599 & TraceONB & Type $2, k=19$ \\
\hline 79 & 313 & TraceONB & Type $2, \mathrm{k}=2$ & 123 & 405 & 3,41 & Thm 2.5.1 \\
\hline 80 & 765 & 5,16 & Thm 2.5.1 & 124 & 489 & TraceONB & Type $1, k=3$ \\
\hline 81 & 161 & Optimal & {$[31]$} & 125 & 745 & TraceONB & Type $2, \mathrm{k}=3$ \\
\hline 82 & 163 & Optimal & {$[31]$} & 126 & 459 & 9,14 & Thm 2.5.1 \\
\hline 83 & 165 & Optimal & {$[31]$} & 127 & 505 & TraceONB & Type $2, k=2$ \\
\hline
\end{tabular}




\begin{tabular}{|c|c|c|c|c|c|c|c|}
\hline$n$ & $\min c_{N}$ & Property & Method & $n$ & $\min c_{N}$ & Property & Method \\
\hline 128 & 7821 & Prime Power & Random & 188 & 1115 & TraceONB & Туре $1, \mathrm{k}=5$ \\
\hline 129 & 1281 & TraceONB & Type $2, \mathrm{k}=5$ & 189 & 377 & Optimal & {$[31]$} \\
\hline 130 & 259 & Optimal & {$[31]$} & 190 & 567 & 2,95 & Thm 2.5.1 \\
\hline 131 & 261 & Optimal & {$[31]$} & 191 & 381 & Optimal & [31] \\
\hline 132 & 455 & 4,33 & Thm 2.5.1 & 192 & 9145 & 3,64 & Thm 2.5.1 \\
\hline 133 & 2223 & 7,19 & Thm 2.5.1 & 193 & 769 & TraceONB & Type $2, \mathrm{k}=2$ \\
\hline 134 & 267 & Optimal & {$[31]$} & 194 & 387 & Optimal & {$[31]$} \\
\hline 135 & 269 & Optimal & [31] & 195 & 645 & 3,65 & Thm 2.5.1 \\
\hline 136 & 1701 & 8,17 & Thm 2.5.1 & 196 & 391 & Optimal & {$[31]$} \\
\hline 137 & 817 & TraceONB & Type $2, \mathrm{k}=3$ & 197 & 3529 & TraceONB & Type $2, \mathrm{k}=9$ \\
\hline 138 & 275 & Optimal & {$[31]$} & 198 & 591 & 2,99 & Thm 2.5.1 \\
\hline 139 & 553 & TraceONB & Type $2, \mathrm{k}=2$ & 199 & 793 & TraceONB & Type $2, \mathbf{k}=2$ \\
\hline 140 & 483 & 4,35 & Thm 2.5.1 & 200 & 1953 & 8,25 & Thm 2.5.1 \\
\hline 141 & 1681 & TraceONB & Type $2, \mathrm{k}=6$ & 201 & 4001 & TraceONB & Type $2, \mathrm{k}=10$ \\
\hline 142 & 847 & TraceONB & Type $2, \mathrm{k}=3$ & 202 & 1207 & TraceONB & Type 2, $\mathrm{k}=3$ \\
\hline 143 & 853 & TraceONB & Type $2, \mathrm{k}=3$ & 203 & 1083 & 7,29 & Thm 2.5.1 \\
\hline 144 & 1445 & 9,16 & Thm 2.5.1 & 204 & 707 & 4,51 & Thm 2.5.1 \\
\hline 145 & 513 & 5,29 & Thm 2.5.1 & 205 & 729 & 5,41 & Thm 2.5.1 \\
\hline 146 & 291 & Optimal & {$[31]$} & 206 & 817 & TraceONB & Type $1, \mathrm{k}=3$ \\
\hline 147 & 877 & TraceONB & Type $2, \mathrm{k}=3$ & 207 & 765 & 9,23 & Thm 2.5.1 \\
\hline 148 & 295 & Optimal & {$[31]$} & 208 & 3825 & 13,16 & Thm 2.5.1 \\
\hline 149 & 2073 & TraceONB & Type $2, \mathrm{k}=7$ & 209 & 417 & Optimal & {$[31]$} \\
\hline 150 & 495 & 3,50 & Thm 2.5.1 & 210 & 419 & Optimal & [31] \\
\hline 151 & 901 & TraceONB & Type $2, \mathrm{k}=3$ & 211 & 2101 & TraceONB & Type $2, \mathrm{k}=5$ \\
\hline 152 & 2457 & 8,19 & Thm 2.5.1 & 212 & 735 & 4,53 & Thm 2.5.1 \\
\hline 153 & 609 & TraceONB & Type $2, \mathrm{k}=2$ & 213 & 849 & TraceONB & Type $2, \mathrm{k}=2$ \\
\hline 154 & 567 & 11,14 & Thm 2.5.1 & 214 & 2131 & TraceONB & Type $2, \mathrm{k}=5$ \\
\hline 155 & 309 & Optimal & {$[31]$} & 215 & 1285 & TraceONB & Type $2, \mathrm{k}=3$ \\
\hline 156 & 515 & 3,52 & Thm 2.5.1 & 216 & 2961 & 8,27 & Thm 2.5.1 \\
\hline 157 & 1561 & TraceONB & Type $2, \mathrm{k}=5$ & 217 & 1297 & TraceONB & Type $2, \mathrm{k}=3$ \\
\hline 158 & 315 & Optimal & {$[31]$} & 218 & 1295 & TraceONB & Type $1, \mathrm{k}=5$ \\
\hline 159 & 525 & 3,53 & Thm 2.5.1 & 219 & 873 & TraceONB & Type $2, \mathrm{k}=2$ \\
\hline 160 & 3249 & 5,32 & Thm 2.5.1 & 220 & 873 & TraceONB & Type $1, \mathrm{k}=3$ \\
\hline 161 & 855 & 7,23 & Thm 2.5.1 & 221 & 441 & Optimal & {$[31]$} \\
\hline 162 & 323 & Optimal & {$[31]$} & 222 & 735 & 3,74 & Thm 2.5.1 \\
\hline 163 & 649 & TraceONB & Type $2, \mathrm{k}=2$ & 223 & 2665 & TraceONB & Type 2, $\mathbf{k}=6$ \\
\hline 164 & 567 & 4,41 & Thm 2.5.1 & 224 & 6859 & 7,32 & Thm 2.5.1 \\
\hline 165 & 585 & 5,33 & Thm 2.5.1 & 225 & 1581 & 9,25 & Thm 2.5.1 \\
\hline 166 & 495 & 2,83 & Thm 2.5.1 & 226 & 451 & Optimal & {$[31]$} \\
\hline 167 & 2325 & TraceONB & Type $2, \mathrm{k}=7$ & 227 & 5877 & TraceONB & Type $2, \mathrm{k}=13$ \\
\hline 168 & 1995 & 3,56 & Thm 2.5.1 & 228 & 2255 & TraceONB & Type $1, \mathrm{k}=9$ \\
\hline 169 & 673 & TraceONB & Type $2, \mathrm{k}=2$ & 229 & 5017 & TraceONB & Type $2, \mathrm{k}=11$ \\
\hline 170 & 1539 & 10,17 & Thm 2.5.1 & 230 & 459 & Optimal & {$[31]$} \\
\hline 171 & 1989 & 9,19 & Thm 2.5.1 & 231 & 461 & Optimal & [31] \\
\hline 172 & 343 & Optimal & {$[31]$} & 232 & 1197 & 8,29 & Thm 2.5.1 \\
\hline 173 & 345 & Optimal & [31] & 233 & 465 & Optimal & [31] \\
\hline 174 & 347 & Optimal & {$[31]$} & 234 & 867 & 9,26 & Thm 2.5.1 \\
\hline 175 & 697 & TraceONB & Type $2, \mathrm{k}=2$ & 235 & 937 & TraceONB & Type $2, \mathrm{k}=2$ \\
\hline 176 & 1785 & 11,16 & Thm 2.5.1 & 236 & 937 & TraceONB & Type $1, \mathrm{k}==3$ \\
\hline 177 & 705 & TraceONB & Type $2, \mathrm{k}=2$ & 237 & 2361 & TraceONB & Type $2, \mathrm{k}=5$ \\
\hline 178 & 355 & Optimal & {$[31]$} & 238 & 711 & 2,119 & Thm 2.5.1 \\
\hline 179 & 357 & Optimal & {$[31]$} & 239 & 477 & Optimal & [31] \\
\hline 180 & 359 & Optimal & {$[31]$} & 240 & 3825 & 3,80 & Thm 2.5.1 \\
\hline 181 & 1081 & TraceONB & Type $2, \mathrm{k}=3$ & 241 & 1441 & TraceONB & Type $2, \mathrm{k}=3$ \\
\hline 182 & 721 & TraceONB & Type $1, \mathrm{k}=3$ & 242 & 1447 & TraceONB & Type $2, \mathrm{k}=3$ \\
\hline 183 & 365 & Optimal & {$[31]$} & 243 & 485 & Optimal & [31] \\
\hline 184 & 945 & 8,23 & Thm 2.5.1 & 244 & 4825 & TraceONB & Type $1, \mathrm{k}=19$ \\
\hline 185 & 2209 & TraceONB & Type $2, \mathrm{k}=6$ & 245 & 489 & Optimal & [31] \\
\hline 186 & 371 & Optimal & {$[31]$} & 246 & 815 & 3,82 & Thm 2.5.1 \\
\hline 187 & 1117 & TraceONB & Type $2, \mathrm{k}=3$ & 247 & 1477 & TraceONB & Type $2, \mathrm{k}=3$ \\
\hline
\end{tabular}




\begin{tabular}{|c|c|c|c|c|c|c|c|}
\hline$n$ & $\min c_{N}$ & Property & Method & $\bar{n}$ & $\min c_{N}$ & Property & Method \\
\hline 248 & 4977 & 8,31 & Thm 2.5.1 & 308 & 1155 & 11,28 & Thm 2.5.1 \\
\hline 249 & 825 & 3,83 & Thm 2.5.1 & 309 & 617 & Optimal & {$[31]$} \\
\hline 250 & 5479 & TraceONB & Type 2, $\mathrm{k}=11$ & 310 & 927 & 2,155 & Thm 2.5.1 \\
\hline 251 & 501 & Optimal & [31] & 311 & 1861 & TraceONB & Type $2, \mathrm{k}=3$ \\
\hline 252 & 935 & 9,28 & Thm 2.5.1 & 312 & 1617 & 8,39 & Thm 2.5.1 \\
\hline 253 & 945 & 11,23 & Thm 2.5.1 & 313 & 1873 & TraceONB & Type $2, \mathrm{k}=3$ \\
\hline 254 & 507 & Optimal & {$[31]$} & 314 & 1871 & TraceONB & Tyре $1, \mathrm{k}=5$ \\
\hline 255 & 909 & 5,51 & Thm 2.5.1 & 315 & 1173 & 9,35 & Thm 2.5.1 \\
\hline 256 & 32033 & Prime Power & Random & 316 & 631 & Optimal & {$[31]$} \\
\hline 257 & 1537 & TraceONB & Type $2, \mathrm{k}=3$ & 317 & 8217 & TraceONB & 'Type $2, \mathrm{k}=13$ \\
\hline 258 & 855 & 3,86 & Thm 2.5.1 & 318 & 1055 & 3,106 & Thm 2.5.1 \\
\hline 259 & 2581 & TraceONB & Type 2, $\mathrm{k}=\mathbf{5}$ & 319 & 1197 & 11,29 & Thm 2.5.1 \\
\hline 260 & 903 & 4,65 & Thm 2.5.1 & 320 & 16461 & 5,64 & Thm 2.5.1 \\
\hline 261 & 521 & Optimal & {$[31]$} & 321 & 3841 & TraceONB & Type 2, $k=6$ \\
\hline 262 & 783 & 2,131 & Thm 2.5.1 & 322 & 1215 & 14,23 & Thm 2.5.1 \\
\hline 263 & 5241 & TraceONB & Type $2, \mathrm{k}=10$ & 323 & 645 & Optimal & {$[31]$} \\
\hline 264 & 1365 & 8,33 & Thm 2.5.1 & 324 & 1127 & 4,81 & Thm 2.5.1 \\
\hline 265 & 945 & 5,53 & Thm 2.5.1 & 325 & 1297 & TraceONB & Type $2, \mathrm{k}=2$ \\
\hline 266 & 3159 & 14,19 & Thm 2.5.1 & 326 & 651 & Optimal & {$[31]$} \\
\hline 267 & 885 & 3,89 & Thm 2.5.1 & 327 & 14345 & TraceONB & Type $2, \mathrm{k}=22$ \\
\hline 268 & 535 & Optimal & {$[31]$} & 328 & 1701 & 8,41 & Thm 2.5.1 \\
\hline 269 & 3753 & TraceONB & Type $2, \mathrm{k}=7$ & 329 & 657 & Optimal & {$[31]$} \\
\hline 270 & 539 & Optimal & {$[31]$} & 330 & 659 & Optimal & {$[31]$} \\
\hline 271 & 2701 & TraceONB & Type $2, \mathbf{k}=5$ & 331 & 1981 & TraceONB & Type $2, \mathrm{k}=3$ \\
\hline 272 & 6885 & 16,17 & Thm 2.5.1 & 332 & 1155 & 4,83 & Thm 2.5.1 \\
\hline 273 & 545 & Optimal & {$[31]$} & 333 & 8721 & 9,37 & Thm 2.5.1 \\
\hline 274 & 2731 & TraceONB & Type $2, \mathbf{k}=5$ & 334 & 2653 & TraceONB & Type $1, k=7$ \\
\hline 275 & 1953 & 11,25 & Thm 2.5.1 & 335 & 4009 & TraceONB & Type $2, \mathrm{k}=6$ \\
\hline 276 & 959 & 4,69 & Thm 2.5.1 & 336 & 8075 & 3,112 & Thm 2.5.1 \\
\hline 277 & 1105 & TraceONB & Type $2, \mathrm{k}=2$ & 337 & 3361 & TraceONB & Type $2, \mathrm{k}=5$ \\
\hline 278 & 555 & Optimal & {$[31]$} & 338 & 675 & Optimal & {$[31]$} \\
\hline 279 & 1113 & TraceONB & Type $2, \mathrm{k}=2$ & 339 & 1125 & 3,113 & Thm 2.5.1 \\
\hline 280 & 1449 & 8,35 & Thm 2.5.1 & 340 & 5103 & 4,85 & Thm 2.5.1 \\
\hline 281 & 561 & Optimal & {$[31]$} & 341 & 4081 & TraceONB & Type $2, \mathrm{k}=6$ \\
\hline 282 & 1687 & TraceONB & Type $2, \mathrm{k}=3$ & 342 & 2047 & TraceONB & Type $2, \mathrm{k}=3$ \\
\hline 283 & 11845 & TraceONB & Type $2, \mathrm{k}=21$ & 343 & 1369 & TraceONB & Type $2, \mathrm{k}=2$ \\
\hline 284 & 1129 & TraceONB & Type $1, \mathrm{k}=3$ & 344 & 15771 & 8,43 & Thm 2.5.1 \\
\hline 285 & 945 & 3,95 & Thm 2.5.1 & 345 & 1233 & 5,69 & Thm 2.5.1 \\
\hline 286 & 1071 & 11,26 & Thm 2.5.1 & 346 & 691 & Optimal & {$[31]$} \\
\hline 287 & 1539 & 7,41 & Thm 2.5.1 & 347 & 2077 & TraceONB & Type 2, $\mathrm{k}=3$ \\
\hline 288 & 6137 & 9,32 & Thm 2.5.1 & 348 & 695 & Optimal & {$[31]$} \\
\hline 289 & 3457 & TraceONB & Type $2, \mathrm{k}=6$ & 349 & 3481 & TraceONB & Type $2, \mathrm{k}=5$ \\
\hline 290 & 1035 & 5,58 & Thm 2.5.1 & 350 & 699 & Optimal & {$[31]$} \\
\hline 291 & 1741 & TraceONB & Type $2, \mathrm{k}=3$ & 351 & 3501 & TraceONB & Type 2, $\mathrm{k}=5$ \\
\hline 292 & 583 & Optimal & {$[31]$} & 352 & 7581 & 11,32 & Thm 2.5.1 \\
\hline 293 & 585 & Optimal & [31] & 353 & 4929 & TraceONB & Type $2, \mathrm{k}=7$ \\
\hline 294 & 975 & 3,98 & Thm 2.5.1 & 354 & 707 & Optimal & {$[31]$} \\
\hline 295 & 6469 & TraceONB & Type $2, \mathrm{k}=11$ & 355 & 2125 & TraceONB & Type $2, \mathrm{k}=3$ \\
\hline 296 & 10773 & 8,37 & Thm 2.5.1 & 356 & 1239 & 4,89 & Thm 2.5.1 \\
\hline 297 & 1777 & TraceONB & Type $2, \mathrm{k}=3$ & 357 & 1185 & 3,119 & Thm 2.5.1 \\
\hline 298 & 2955 & TraceONB & Type $1, \mathrm{k}=9$ & 358 & 1071 & 2,179 & Thm 2.5.1 \\
\hline 299 & 597 & Optimal & [31] & 359 & 717 & Optimal & [31] \\
\hline 300 & 995 & 3,100 & Thm 2.5.1 & 360 & 3213 & 5,72 & Thm 2.5.1 \\
\hline 301 & 3001 & TraceONB & Type 2, $\mathrm{k}=5$ & 361 & 10801 & TraceONB & Type $2, k=15$ \\
\hline 302 & 1201 & TraceONB & Type $1, \mathrm{k}=3$ & 362 & 10831 & TraceONB & Type $2, \mathrm{k}=15$ \\
\hline 303 & 605 & Optimal & [31] & 363 & 1449 & TraceONB & Type $2, k=2$ \\
\hline 304 & 9945 & 16,19 & Thm 2.5.1 & 364 & 1957 & 7,52 & Thm 2.5.1 \\
\hline 305 & 6081 & TraceONB & Type $2, \mathrm{k}=10$ & 365 & 9465 & TraceONB & Type $2, \mathrm{k}=13$ \\
\hline 306 & 611 & Optimal & {$[31]$} & 366 & 1095 & 2,183 & Thm 2.5.1 \\
\hline 307 & 1225 & TraceONB & Type $2, \mathrm{k}=2$ & 367 & 3661 & TraceONB & Type $2, \mathrm{k}=5$ \\
\hline
\end{tabular}




\begin{tabular}{|c|c|c|c|c|c|c|c|}
\hline$n$ & $\min c_{N}$ & Property & Method & $n$ & $\min c_{N}$ & Property & Method \\
\hline 368 & 3825 & 16,23 & Thm 2.5.1 & 428 & 2555 & TraceONB & Type $1, \bar{k}=5$ \\
\hline 369 & 1377 & 9,41 & Thm 2.5.1 & 429 & 857 & Optimal & {$[31]$} \\
\hline 370 & 1323 & 5,74 & Thm 2.5.1 & 430 & 1539 & 5,86 & Thm 2.5.1 \\
\hline 371 & 741 & Optimal & {$[31]$} & 431 & 861 & Optimal & {$[31]$} \\
\hline 372 & 743 & Optimal & [31] & 432 & 11985 & 16,27 & Thm 2.5.1 \\
\hline 373 & 1489 & TraceONB & Type $2, \mathbf{k}=\mathbf{2}$ & 433 & 1729 & TraceONB & Type $2, \mathbf{k}=2$ \\
\hline 374 & 1489 & TraceONB & Type $1, \mathbf{k}=\mathbf{3}$ & 434 & 4315 & TraceONB & Type $1, \mathbf{k}=9$ \\
\hline 375 & 749 & Optimal & {$[31]$} & 435 & 1737 & TraceONB & Type $2, \mathrm{k}=2$ \\
\hline 376 & 19173 & 8,47 & Thm 2.5.1 & 436 & 14727 & TraceONB & Type $1, \mathrm{k}=33$ \\
\hline 377 & 2565 & 13,29 & Thm 2.5.1 & 437 & 5265 & 19,23 & Thm 2.5.1 \\
\hline 378 & 755 & Optimal & {$[31]$} & 438 & 875 & Optimal & [31] \\
\hline 379 & 6805 & TraceONB & Type 2, k $=9$ & 439 & 4381 & TraceONB & Type $2, \mathrm{k}=5$ \\
\hline 380 & 1323 & 4,95 & Thm 2.5.1 & 440 & 3969 & 5,88 & Thm 2.5.1 \\
\hline 381 & 7601 & TraceONB & Type $2, \mathrm{k}=10$ & 441 & 881 & Optimal & [31] \\
\hline 382 & 1143 & 2,191 & Thm 2.5.1 & 442 & 883 & Optimal & {$[31]$} \\
\hline 383 & 16045 & TraceONB & Type $2, k=21$ & 443 & 885 & Optimal & {$[31]$} \\
\hline 384 & 39105 & 3,128 & Thm 2.5.1 & 444 & 1475 & 3,148 & Thm 2.5.1 \\
\hline 385 & 1449 & 11,35 & Thm 2.5.1 & 445 & 1593 & 5,89 & Thm 2.5.1 \\
\hline 386 & 771 & Optimal & {$[31]$} & 446 & 2671 & TraceONB & Type $2, \mathrm{k}=3$ \\
\hline 387 & 1545 & TraceONB & Type $2, \mathrm{k}=2$ & 447 & 2677 & TraceONB & Type $2, \mathrm{k}=3$ \\
\hline 388 & 775 & Optimal & {$[31]$} & 448 & 34751 & 7,64 & Thm 2.5.1 \\
\hline 389 & 14745 & TraceONB & Type $2, \mathrm{k}=19$ & 449 & 6273 & TraceONB & Type $2, \mathrm{k}=7$ \\
\hline 390 & 1295 & 3,130 & Thm 2.5.1 & 450 & 1683 & 9,50 & Thm 2.5.1 \\
\hline 391 & 3645 & 17,23 & Thm 2.5.1 & 451 & 1701 & 11,41 & Thm 2.5.1 \\
\hline 392 & 21021 & 8,49 & Thm 2.5.1 & 452 & 1575 & 4,113 & Thm 2.5.1 \\
\hline 393 & 785 & Optimal & {$[31]$} & 453 & 905 & Optimal & {$[31]$} \\
\hline 394 & 3915 & TraceONB & Type $1, \mathbf{k}=\mathbf{9}$ & 454 & 9025 & TraceONB & Type $1, \mathrm{k}=19$ \\
\hline 395 & 2365 & TraceONB & Type $2, \mathrm{k}=\mathbf{3}$ & 455 & 2451 & 7,65 & Thm 2.5.1 \\
\hline 396 & 1379 & 4,99 & Thm 2.5.1 & 456 & 12285 & 3,152 & Thm 2.5.1 \\
\hline 397 & 22969 & TraceONB & Type $2, k=29$ & 457 & 13681 & TraceONB & Type $2, \mathrm{k}=15$ \\
\hline 398 & 795 & Optimal & {$[31]$} & 458 & 11883 & TraceONB & Type $2, \mathrm{k}=13$ \\
\hline 399 & 4777 & TraceONB & Type $2, \mathrm{k}=6$ & 459 & 4581 & TraceONB & Type $2, \mathrm{k}=\mathbf{5}$ \\
\hline 400 & 7905 & 16,25 & Thm 2.5.1 & 460 & 919 & Optimal & {$[31]$} \\
\hline 401 & 4801 & TraceONB & Type $2, k=6$ & 461 & 9201 & TraceONB & Type $2, \mathrm{k}=10$ \\
\hline 402 & 1335 & 3,134 & Thm 2.5.1 & 462 & 1383 & 2,231 & Thm 2.5.1 \\
\hline 403 & 8845 & TraceONB & Type $2, k=11$ & 463 & 5545 & 'TraceONB & Type $2, \mathrm{k}=6$ \\
\hline 404 & 1609 & TraceONB & Type $1, \mathrm{k}=\mathbf{3}$ & 464 & 4845 & 16,29 & Thm 2.5.1 \\
\hline 405 & 1449 & 5,81 & Thm 2.5.1 & 465 & 1545 & 3,155 & Thm 2.5.1 \\
\hline 406 & 1539 & 14,29 & Thm 2.5.1 & 466 & 931 & Optimal & {$[31]$} \\
\hline 407 & 10773 & 11,37 & Thm 2.5.1 & 467 & 2797 & TraceONB & Type $2, \mathrm{k}=3$ \\
\hline 408 & 2121 & 8,51 & Thm 2.5 .1 & 468 & 1751 & 9,52 & Thm 2.5.1 \\
\hline 409 & 1633 & TraceONB & Type $2, \mathrm{k}=2$ & 469 & 1873 & TraceONB & Type $2, \mathbf{k}=2$ \\
\hline 410 & 819 & Optimal & {$[31]$} & 470 & 939 & Optimal & {$[31]$} \\
\hline 411 & 821 & Optimal & {$[31]$} & 471 & 9401 & TraceONB & Type $2, \mathrm{k}=10$ \\
\hline 412 & 1641 & TraceONB & Type $1, \mathrm{k}=3$ & 472 & 32067 & 8,59 & Thm 2.5.1 \\
\hline 413 & 825 & Optimal & {$[31]$} & 473 & 945 & Optimal & {$[31]$} \\
\hline 414 & 827 & Optimal & {$[31]$} & 474 & 1575 & 3,158 & Thm 2.5.1 \\
\hline 415 & 1485 & 5,83 & Thm 2.5.1 & 475 & 1897 & TraceONB & Type $2, \mathrm{k}=2$ \\
\hline 416 & 16245 & 13,32 & Thm 2.5.1 & 476 & 1659 & 4,119 & Thm 2.5.1 \\
\hline 417 & 1665 & TraceONB & Type $2, k=2$ & 477 & 1785 & 9,53 & Thm 2.5.1 \\
\hline 418 & 835 & Optimal & [31] & 478 & 1431 & 2,239 & Thm 2.5.1 \\
\hline 419 & 837 & Optimal & {$[31]$} & 479 & 5737 & TraceONB & Type $2, \mathrm{k}=6$ \\
\hline 420 & 839 & Optimal & {$[31]$} & 480 & 16245 & 3,160 & Thm 2.5.1 \\
\hline 421 & 11761 & TraceONB & Type $2, \mathrm{k}=14$ & 481 & 2881 & TraceONB & Type $2, \mathrm{k}=3$ \\
\hline 422 & 10947 & TraceONB & Type $2, k=13$ & 482 & 8659 & TraceONB & Type $2, \mathrm{k}=9$ \\
\hline 423 & 1689 & TraceONB & Type $2, \mathrm{k}=2$ & 483 & 965 & Optimal & {$[31]$} \\
\hline 424 & 2205 & 8,53 & Thm 2.5.1 & 484 & 1929 & TraceONB & Type $1, \mathrm{k}=3$ \\
\hline 425 & 2545 & TraceONB & Type $2, \mathrm{k}=3$ & 485 & 8713 & TraceONB & Type $2, \mathrm{k}=9$ \\
\hline 426 & 851 & Optimal & [31] & 486 & 1455 & 2,243 & Thm 2.5.1 \\
\hline 427 & 7669 & TraceONB & Type $2, \mathbf{k}=\mathbf{9}$ & 487 & 1945 & TraceONB & Type $2, \mathrm{k}=2$ \\
\hline
\end{tabular}




\begin{tabular}{|c|c|c|c|c|c|c|c|}
\hline$n$ & $\min c_{N}$ & Property & Method & $n$ & $\min c_{N}$ & Property & Method \\
\hline 488 & 34041 & 8,61 & Thm 2.5.1 & 548 & 3275 & TraceONB & Type $1, \mathrm{k}=5$ \\
\hline 489 & 5857 & TraceONB & Type $2, \mathrm{k}=6$ & 549 & 7673 & TraceONB & Type $2, \mathrm{k}=7$ \\
\hline 490 & 979 & Optimal & [31] & 550 & 2079 & 11,50 & Thm 2.5.1 \\
\hline 491 & 981 & Optimal & [31] & 551 & 3301 & TraceONB & Type $2, \mathrm{k}=3$ \\
\hline 492 & 1863 & 12,41 & Thm 2.5.1 & 552 & 2877 & 8,69 & Thm 2.5.1 \\
\hline 493 & 1969 & TraceONB & Type $2, \mathrm{k}=2$ & 553 & 2209 & TraceONB & Type 2, $\mathrm{k}=2$ \\
\hline 494 & 1969 & TraceONB & Type $1, \mathrm{k}=\mathbf{3}$ & 554 & 1107 & Optimal & {$[31]$} \\
\hline 495 & 989 & Optimal & {$[31]$} & 555 & 2217 & TraceONB & Type $2, \mathrm{k}=2$ \\
\hline 496 & 20145 & 16,31 & Thm 2.5.1 & 556 & 1111 & Optimal & {$[31]$} \\
\hline 497 & 9921 & TraceONB & Type $2, \mathbf{k}=10$ & 557 & 24465 & TraceONB & Type $2, \mathrm{k}=22$ \\
\hline 498 & 1815 & 6,83 & Thm 2.5.1 & 558 & 1115 & Optimal & [31] \\
\hline 499 & 1993 & TraceONB & Type $2, \mathbf{k}=2$ & 559 & 2233 & TraceONB & Type $2, \mathrm{k}=2$ \\
\hline 500 & 5969 & TraceONB & Type $1, \mathrm{k}=11$ & 560 & 5865 & 16,35 & Thm 2.5.1 \\
\hline 501 & 5001 & TraceONB & Type $2, k=5$ & 561 & 1121 & Optimal & {$[31]$} \\
\hline 502 & 1503 & 2,251 & Thm 2.5.1 & 562 & 1123 & Optimal & {$[31]$} \\
\hline 503 & 3013 & TraceONB & Type $2, \mathbf{k}=3$ & 563 & 7869 & TraceONB & Type $2, \mathbf{k}=7$ \\
\hline 504 & 6783 & 7,72 & Thm 2.5.1 & 564 & 2249 & TraceONB & Type $1, \mathbf{k}=3$ \\
\hline 505 & 5041 & TraceONB & Type $2, \mathrm{k}=5$ & 565 & 2025 & 5,113 & Thm 2.5.1 \\
\hline 506 & 2835 & 2,253 & Thm 2.5.1 & 566 & 14691 & TraceONB & Type $2, \mathbf{k}=13$ \\
\hline 507 & 2025 & TraceONB & Type $2, \mathbf{k}=2$ & 567 & 2265 & TraceONB & Type $2, \mathrm{k}=2$ \\
\hline 508 & 1015 & Optimal & [31] & 568 & 48615 & 8,71 & Thm 2.5 .1 \\
\hline 509 & 1017 & Optimal & [31] & 569 & 6817 & TraceONB & Type $2, k=6$ \\
\hline 510 & 1919 & 10,51 & Thm 2.5.1 & 570 & 2079 & 6,95 & Thm 2.5.1 \\
\hline 511 & 3061 & TraceONB & Type $2, \mathrm{k}=\mathbf{3}$ & 571 & 26221 & TraceONB & Type $2, \mathbf{k}=23$ \\
\hline 512 & 131072 & Prime Power & Random & 572 & 2163 & 11,52 & Thm 2.5.1 \\
\hline 513 & 2049 & TraceONB & Type $2, \mathrm{k}=2$ & 573 & 1905 & 3,191 & Thm 2.5.1 \\
\hline 514 & 17443 & TraceONB & Type $2, \mathrm{k}=17$ & 574 & 2187 & 14,41 & Thm 2.5.1 \\
\hline 515 & 1029 & Optimal & {$[31]$} & 575 & 1149 & Optimal & {$[31]$} \\
\hline 516 & 1715 & 3,172 & Thm 2.5.1 & 576 & 31093 & 9,64 & Thm 2.5.1 \\
\hline 517 & 2065 & TraceONB & Type $2, \mathrm{k}=2$ & 577 & 2305 & TraceONB & Type $2, \mathrm{k}=2$ \\
\hline 518 & 2793 & 7,74 & Thm 2.5.1 & 578 & 3463 & TraceONB & Type $2, \mathrm{k}=3$ \\
\hline 519 & 1037 & Optimal & {$[31]$} & 579 & 5781 & TraceONB & Type $2, \mathrm{k}=5$ \\
\hline 520 & 2709 & 8,65 & Thm 2.5.1 & 580 & 2313 & TraceONB & Туре $1, \mathrm{k}=3$ \\
\hline 521 & 18721 & TraceONB & Type $2, \mathrm{k}=18$ & 581 & 3135 & 7,83 & Thm 2.5.1 \\
\hline 522 & 1043 & Optimal & {$[31]$} & 582 & 1935 & 3,194 & Thm 2.5 .1 \\
\hline 523 & 5221 & TraceONB & Type 2, $\mathrm{k}=\mathbf{5}$ & 583 & 2205 & 11,53 & Thm 2.5.1 \\
\hline 524 & 1827 & 4,131 & Thm 2.5.1 & 584 & 50211 & 8,73 & Thm 2.5.1 \\
\hline 525 & 8835 & 3,175 & Thm 2.5.1 & 585 & 1169 & Optimal & {$[31]$} \\
\hline 526 & 5251 & TraceONB & Type $2, \mathrm{k}=5$ & 586 & 1171 & Optimal & [31] \\
\hline 527 & 18937 & TraceONB & Type $2, \mathrm{k}=18$ & 587 & 8205 & TraceONB & Type $2, \mathrm{k}=7$ \\
\hline 528 & 5525 & 16,33 & Thm 2.5.1 & 588 & 1955 & 3,196 & Thm 2.5.1 \\
\hline 529 & 14785 & TraceONB & Type $2, \mathrm{k}=14$ & 589 & 2353 & TraceONB & Type $2, \mathrm{k}=2$ \\
\hline 530 & 1059 & Optimal & {$[31]$} & 590 & 7049 & TraceONB & Type $1, \mathrm{k}=11$ \\
\hline 531 & 1061 & Optimal & [31] & 591 & 3541 & TraceONB & Type $2, \mathbf{k}=3$ \\
\hline 532 & 5295 & TraceONB & Type $1, \mathrm{k}=\mathbf{9}$ & 592 & 43605 & 16,37 & Thm 2.5.1 \\
\hline 533 & 3645 & 13,41 & Thm 2.5.1 & 593 & 1185 & Optimal & [31] \\
\hline 534 & 1775 & 3,178 & Thm 2.5.1 & 594 & 8883 & 2,297 & Thm 2.5.1 \\
\hline 535 & 2137 & TraceONB & Type $2, \mathrm{k}=2$ & 595 & 2133 & 5,119 & Thm 2.5.1 \\
\hline 536 & 42063 & 8,67 & Thm 2.5.1 & 596 & 10679 & TraceONB & Type $1, \mathrm{k}=17$ \\
\hline 537 & 1785 & 3,179 & Thm 2.5.1 & 597 & 2385 & TraceONB & Type $2, \mathbf{k}=2$ \\
\hline 538 & 24703 & TraceONB & Type $2, \mathrm{k}=23$ & 598 & 1791 & 2,299 & Thm 2.5.1 \\
\hline 539 & 6457 & TraceONB & Type $2, \mathbf{k}=6$ & 599 & 8373 & TraceONB & Type $2, k=7$ \\
\hline 540 & 1079 & Optimal & [31] & 600 & 9765 & 3,200 & Thm 2.5.1 \\
\hline 541 & 11881 & TraceONB & Type $2, \mathrm{k}=11$ & 601 & 6001 & TraceONB & Type $2, \mathrm{k}=5$ \\
\hline 542 & 3247 & TraceONB & Type $2, \mathrm{k}=3$ & 602 & 3249 & 7,86 & Thm 2.5.1 \\
\hline 543 & 1085 & Optimal & [31] & 603 & 7225 & TraceONB & Type 2, $\mathrm{k}=6$ \\
\hline 544 & 29241 & 17,32 & Thm 2.5.1 & 604 & 4813 & TraceONB & Type $1, k=7$ \\
\hline 545 & 1089 & Optimal & {$[31]$} & 605 & 10873 & TraceONB & Type $2, \mathbf{k}=9$ \\
\hline 546 & 1091 & Optimal & {$[31]$} & 606 & 1211 & Optimal & {$[31]$} \\
\hline 547 & 25117 & TraceONB & Type $2, \mathrm{k}=23$ & 607 & 3637 & TraceONB & Type 2, $\mathrm{k}=3$ \\
\hline
\end{tabular}




\section{Appendix D}

\section{Low-Complexity Subfields}

Type I $q$ even, $m=n / 4$

\begin{tabular}{|c|c|c|c|c|c|}
\hline$n$ & $m=n / 4$ & $C_{m}=4 m-3$ & $n$ & $m=n / 4$ & $C_{m}=4 m-3$ \\
\hline 52 & 13 & 49 & 612 & 153 & 609 \\
\hline 60 & 15 & 57 & 652 & 163 & 649 \\
\hline 100 & 25 & 97 & 660 & 165 & 657 \\
\hline 148 & 37 & 145 & 676 & 169 & 673 \\
\hline 172 & 43 & 169 & 700 & 175 & 697 \\
\hline 180 & 45 & 177 & 708 & 177 & 705 \\
\hline 196 & 49 & 193 & 756 & 189 & 753 \\
\hline 268 & 67 & 265 & 772 & 193 & 769 \\
\hline 292 & 73 & 289 & 796 & 199 & 793 \\
\hline 316 & 79 & 313 & 820 & 205 & 817 \\
\hline 348 & 87 & 345 & 828 & 207 & 825 \\
\hline 372 & 93 & 369 & 852 & 213 & 849 \\
\hline 388 & 97 & 385 & 876 & 219 & 873 \\
\hline 420 & 105 & 417 & 940 & 235 & 937 \\
\hline 460 & 115 & 457 & 1060 & 265 & 1057 \\
\hline 508 & 127 & 505 & 1108 & 277 & 1105 \\
\hline 540 & 135 & 537 & 1116 & 279 & 1113 \\
\hline 556 & 139 & 553 & & & \\
\hline
\end{tabular}

Type II $m=n / 2$

\begin{tabular}{|r|r|r|r|r|r|}
\hline$n$ & $m=n / 2$ & $C_{m}=4 m-3$ & $n$ & $m=n / 2$ & $C_{m}=4 m-3$ \\
\hline 278 & 139 & 553 & 650 & 325 & 1297 \\
306 & 153 & 609 & 686 & 343 & 1369 \\
326 & 163 & 649 & 690 & 345 & 1377 \\
330 & 165 & 657 & 726 & 363 & 1449 \\
338 & 169 & 673 & 746 & 373 & 1489 \\
350 & 175 & 697 & 774 & 387 & 1545 \\
354 & 177 & 705 & 810 & 405 & 1617 \\
386 & 193 & 769 & 818 & 409 & 1633 \\
398 & 199 & 793 & 834 & 417 & 1665 \\
410 & 205 & 817 & 846 & 423 & 1689 \\
414 & 207 & 825 & 866 & 433 & 1729 \\
426 & 213 & 849 & 870 & 435 & 1737 \\
438 & 219 & 873 & 930 & 465 & 1857 \\
470 & 235 & 937 & 938 & 469 & 1873 \\
530 & 265 & 1057 & 950 & 475 & 1897 \\
554 & 277 & 1105 & 974 & 487 & 1945 \\
558 & 279 & 1113 & 986 & 493 & 1969 \\
614 & 307 & 1225 & 998 & 499 & 1993 \\
638 & 319 & 1273 & 1026 & 513 & 2049 \\
\hline
\end{tabular}




\section{Bibliography}

[1] D. W. Ash, I. F. Blake, and S. A Vanstone, Low complexity normal bases, Discrete Applied Mathematics 25 (1989), 191-210.

[2] P. S. L. M Barreto, A note on efficient computation of cube roots in characteristic 3, Cryptology ePrint Archive (2004), no. 305.

[3] I. F. Blake, S. Gao, and R. C. Mullin, Normal and self-dual normal bases from factorization of $c x^{q+1}+d x^{q}-a x-b$, SIAM Journal on Discrete Mathematics 7 (1994), 499-512.

[4] M. Christopoulou, T. Garefalakis, D. Panario, and D. Thomson, The trace of an optimal normal element and low complexity normal bases, Submitted (2007).

[5] S. D. Cohen, Explicit theorems on generator polynomials, Finite Fields and Their Applications 11 (2005), 337-357.

[6] D. Dummit and R. Foote, Abstract Algebra, 2nd ed., John Wiley and Sons, Toronto, 1999.

[7] I. Duursma and H. S. Lee, Tate pairing implementation for hyperelliptic curves $y^{2}=x^{p}-x+d$, Advances in Cryptology - ASIACRYPT 20032894 (2003), 111-123.

[8] M. Ernst, M. Jung, F. Madlener, S. Huss, and R. Blümel, A reconfigurable system on chip implementation for elliptic curve cryptography over $\mathbb{G} \mathbb{F}\left\{2^{n}\right\}$, Lecture Notes on Computer Science 2523 (2002), 381-399.

[9] G. S. Fransden, On the density of normal basis in finite fields, Finite Fields and Their Applications 6 (2000), 23-38.

[10] S. Gao, Normal Bases over Finite Fields, Ph.D Thesis, Waterloo, ON, 1993.

[11] S. Gao and D. Panario, Density of normal elements, Finite Fields and Their Applications 3 (1997), 141-150.

[12] S. Gao and H. W. Lenstra, Optimal normal bases, Designs, Codes and Cryptography 2 (1992), 315-323.

[13] J. von zur Gathen, Irreducible trinomials over finite fields, Mathematics of Computation 72 (2003), no. 244, 1987-2000.

[14] J. von zur Gathen and J. Gerhard, Modern Computer Algebra, 2nd edition, Cambridge University Press, Cambridge, UK, 2003.

$[15] \ldots$ _ Polynomial factorization over $\mathbb{F}_{2}$, Mathematics of Computation 71 (2002), 1677-1698.

[16] J. von zur Gathen and M. Giesbrecht, Constructing normal bases in finite fields, Journal of Symbolic Computation 10 (1990), 547-570.

[17] W. Geiselmann, Algebraische Algorithmenentwicklung am Beispiel der Arithmetik in endlichen Körpern, Dissertation, Universität Karlsruhe, 1992. 
[18] A. Hales and D. Newhart, Swan's theorem for binary tetranomials, Finite Fields and Their Applications 12 (2006), no. 2, 306-311.

[19] K. Hensel, Uber die Darstellung der Zahlen eines Gattungsbereiches für einen beliebigen Primdivisor, Journal für die reine und angewandte Mathematik 103 (1888), 230-237.

[20] K. Hoffman and R. Kunze, Linear Algebra, Prentice Hall, New Jersey, 1961.

[21] Standard Specifications for Public-Key Cryptography, IEEE, IEEE Inc., 3 Park Ave., NY 100165997, USA.

[22] D. Jungnickel, Finite Fields: Structure and Arithmetics, B.I. Wissenschaftsverlag, Mannheim, Germany, 1993.

[23] A. Karatsuba and Y. Ofman, Multiplication of Multidigit Numbers on Automata, Soviet Physics Doklady 7 (1963), 595-596.

[24] H. W. Lenstra Jr. and R. J. Schoof, Primitive normal bases for finite fields, Mathematics of Computation 48 (1987), no. 177, 217-231.

[25] R. Lidl and H. Neiderreither, Finite fields, 2nd ed., Cambridge University Press, Cambridge, UK, 1997.

[26] A. Reyhani-Masoleh and A. Hasan, Low complexity word-level sequential normal-basis multipliers, IEEE Transactions on Computers 54 (2005), 98-110.

[27] A. Masuda, L. Moura, D. Panario, and D. Thomson, Low complexity of normal elements over finite fields of characteristic two, Submitted (2006).

[28] M. Mendez and A. Pala, Type I error rate and power of three normality tests, Pakistan Journal of Information and Technology 2 (2003), 135-139.

[29] F. Meyer, Normalbasismultiplikation in endlichen Körpern, Ph.D Thesis, University of Karlsruhe, 1990.

[30] P. Moree, Artin's primitive root conjecture: a survey arXiv:math/0412262v1 (2004), available at http://arxiv.org/abs/math/0412262v1.

[31] R. C. Mullin, I. M. Onyszchuk, S. A. Vanstone, and R. M. Wilson, Optimal normal bases in $G F\left(p^{n}\right)$, Discrete Applied Mathematics 22 (1988/1989), 149-161.

[32] R. Murty, Artin's conjecture for primitive roots, The Mathematical Intelligencer 10 (1988), no. 4, 59-67.

[33] NIST, Recommended Elliptic Curves for Federal Government Use, 1999, http://csrc.nist. gov/CryptoToolkit/dss/ecdsa/NISTReCur.pdf.

[34] C. Savage, A survey of combinatorial Gray codes, SIAM Review 39 (1997), 605-629.

[35] G. E. Seguin, Low complexity normal bases for $\mathbb{F}_{2}^{m n}$, Discrete Applied Mathematics 28 (1990), 309-312.

[36] V. Shoup, NTL: A Library for doing Number Theory, http://www.shoup.net/ntl, 2005.

[37] N. P. Smart, Elliptic Curve Cryptosystems over Small Fields of Odd Characteristic, Journal of Cryptology 12 (1999), 141-151. 